\title{
WEIGHTED UPWINDING COMPACT SCHEME FOR SHOCK CAPTURING
}

\author{
by \\ HASSAN ABD SALMAN AL-DUJALY
}

Presented to the Faculty of the Graduate School of

The University of Texas at Arlington in Partial Fulfillment

of the Requirements

for the Degree of

DOCTOR OF PHILOSOPHY

THE UNIVERSITY OF TEXAS AT ARLINGTON

May 2017 
Copyright $@$ by HASSAN ABD SALMAN AL-DUJALY 2017

All Rights Reserved 


\section{ACKNOWLEDGEMENTS}

First and above all, thanks to Allah for all the blessings I have received from him and for providing me the opportunity to complete this dissertation.

Also, I would like to offer my sincere thanks to everyone who helped and supported me in completing this work. I submit my warmest and deepest appreciation to my research advisor and committee chairman, Dr. Chaoqun Liu, for his insightful discussion, valuable guidance, and unconditional support throughout my PhD program at the University of Texas at Arlington. This appreciation is also dedicated to Dr. Benito Chen-Charpentier, Dr. Hristo V. Kojouharov, Dr. Goujun Liao, and Dr. Ren-Cang Li for serving as my committee members and reviewing my dissertation. Additionally, I am grateful to all faculty and staff in the Department of Mathematics for their help and support. I am also indebted to the Higher Committee for Education Development in Iraq (HCED) for monitoring my progress and providing financial support.

I would like to express my highest gratitude to my tenderhearted father and beloved mother. This is my precious gift to you for all your sacrifices to give me this life. Although I have lived far from you, communication with you provided emotional comfort for me, and your overwhelming encouragement has kept me going. I always keep you close in my mind and my heart. Also, this gratitude is dedicated to my great brothers and sisters for supporting me spiritually throughout writing this dissertation and my life in general. I certainly would like to thank you all for everything. 
I'd be remiss if I didn't acknowledge the innumerable sacrifices made by my wife, Roaa, in taking care of our children, Fatima, Zainab, Hajir, and Sarah, and for her patience, assistance, and continuous support while I pursued the $\mathrm{PhD}$ degree.

Finally, I would like to express my gratitude to my relatives and friends for their spiritual help and support.

April 24,2017 


\begin{abstract}
WEIGHTED UPWINDING COMPACT SCHEME FOR SHOCK CAPTURING
\end{abstract}

\author{
HASSAN ABD SALMAN AL-DUJALY, Ph.D.
}

The University of Texas at Arlington, 2017

Supervising Professor: Dr. Chaoqun Liu

The Weighted Upwinding Compact Scheme in this dissertation has been constructed due to dissipation and dispersion analysis at each stencil. The new scheme is applied to many one-dimensional typical problems involving discontinuities and shock waves, and it maintains a $7^{\text {th }}$ order of accuracy in smooth areas. Additionally, when using the technology of decoupling the system of WUCS, the global dependency problem of the compact scheme is transferred to a local dependency problem in shock regions. As a result of the decoupling method, the shocks are captured sharply with fewer points compared to the related schemes. Furthermore, high order, high resolution, and nonoscillation are achieved.

In future work, there will be an effort to apply the new scheme to the 2-D and 3-D Navier-Stokes equations and to multi-dimensional flows with shock-turbulence interaction. 


\section{TABLE OF CONTENTS}

ACKNOWLEDGEMENTS......................................................................... iii

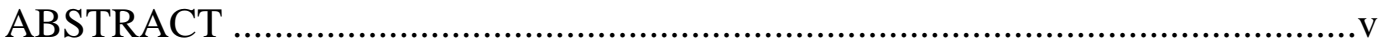

LIST OF ILLUSTRATIONS .................................................................... ix

LIST OF TABLES .............................................................................. xiv

Chapter 1: INTRODUCTION ..................................................................

Chapter 2: MODIFIED BUFFERED FOURIER METHOD ..............................7

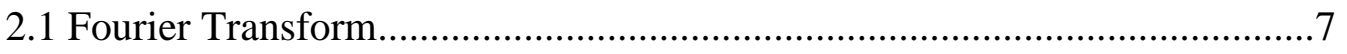

2.1.1 Continuous Fourier Transform ....................................................

2.1.2 Discrete Fourier Transform (DFT) ................................................

2.1.3. Standard Fourier Spectral Method (SFSM) ......................................9

2.2. Modified Buffered Fourier Method (MBFM) .........................................9

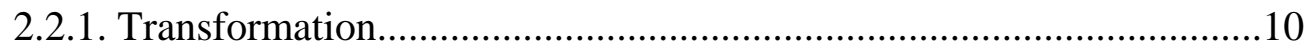

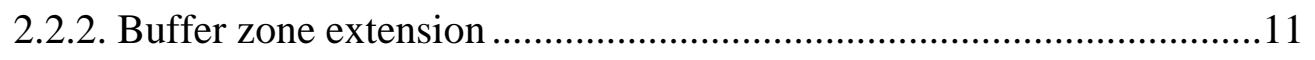

2.2.3. Normalization .......................................................................... 11

2.3. Numerical Results............................................................................ 11

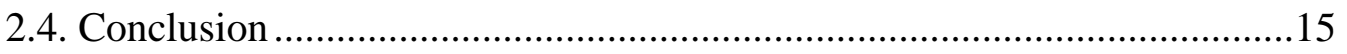

Chapter 3: WEIGHTED UPWINDING COMPACT SCHEME (WUCS)............16

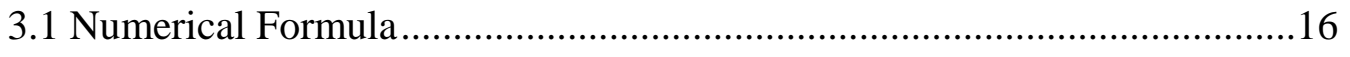

3.1.1. Essentially Non-Oscillatory (ENO) scheme and conservative reconstruction ....................................................16 
3.1.2. $5^{\text {th }}$ order Weighted Essentially Non-Oscillatory (WENO) scheme .....18

3.1.2.1. Flux approximation.....................................................................19

3.1.2.2. Optimal weights for a high order accuracy.................................20

3.1.2.3. Non-linear weights for shock capturing......................................21

3.1.2.4. Boundary points schemes ...........................................................22

3.1.3. The finite difference Compact Scheme (CS) .......................................23

3.1.4. Weighted Compact Scheme (WCS) ……………………......................24

3.1.5. Modified Upwinding Compact Scheme (MUCS) ...................................26

3.1.6. Modified Weighted Compact Scheme (MWCS) ...................................27

3.2. Weighted Upwinding Compact Scheme (WUCS) ………............................27

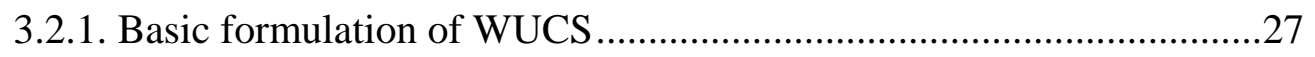

3.2.2. Boundary points schemes ................................................................28

3.3. Dispersion and Dissipation Analysis ............................................................29

3.3.1. Dispersion and dissipation analysis for the left stencils $\left(\mathrm{S}^{0}\right) \ldots \ldots \ldots \ldots \ldots . . . .31$

3.3.2. Dispersion and dissipation analysis for the center stencils $\left(\mathrm{S}^{1}\right)$............35

3.3.2. Dispersion and dissipation analysis for the right stencils $\left(\mathrm{S}^{2}\right)$.............38

3.3.4. Dispersion and dissipation analysis for the overall schemes ................41

3.4. Decoupling the system of WUCS..............................................................44

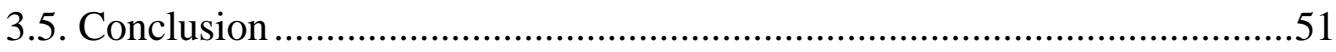

Chapter 4: NUMERICAL RESULTS .............................................................52

4.1. Convection Equation .................................................................................52

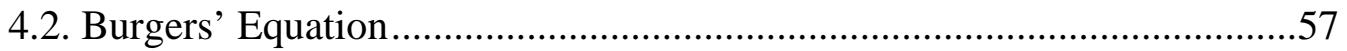


4.3. Euler equations

4.3.1. Shock tube problem .......................................................................60

4.3.2. Shock-entropy interaction problem ..............................................64

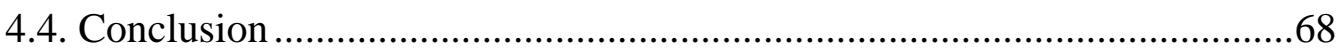

Chapter 5: CONCLUSION AND FUTURE WORK .........................................69

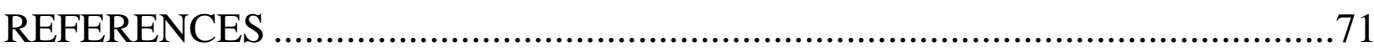

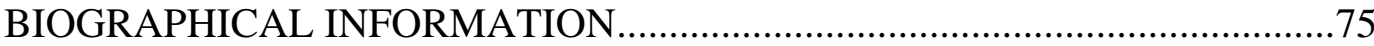




\section{LIST OF ILLUSTRATIONS}

Figure 2.1 The buffer polynomial of 8 points constructed according to the cubic spline interpolation.....................................................12

Figure 2.2 The extended function at the new domain ......................................12

Figure 2.3 The source function after transformation .....................................13

Figure 2.4 The shifted buffered function at the new domain .............................13

Figure 2.5 The transformed source function...................................................14

Figure 2.6 The shifted buffered function at the new domain ...........................14

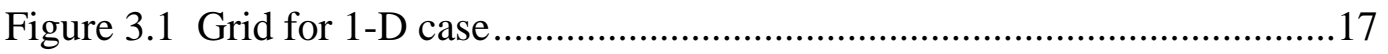

Figure 3.2 The stencils for the WENO scheme .............................................19

Figure 3.3 1-D grid for the compact scheme ..............................................23

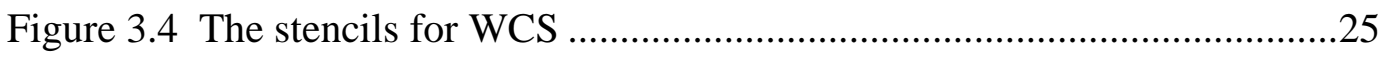

Figure 3.5 The dispersion in the left stencils of wucs,

WCS, and the WENO scheme ..............................................34

Figure 3.6 Dissipation in the left stencils of WUCS,

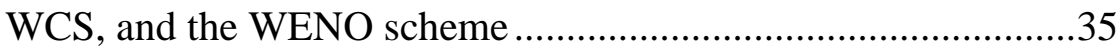

Figure 3.7 The dispersion in the center stencils of WUCS,

WCS, and the WENO scheme ............................................37

Figure 3.8 The dissipation in the center stencils of WUCS,

WCS, and the WENO scheme .38

Figure 3.9 The dispersion in the right stencils of WUCS,

WCS, and the WENO scheme .40 
Figure 3.10 The dissipation in the right stencils of WUCS,

WCS, and the WENO scheme …………………………………....

Figure 3.11 The dispersion in WUCS, WCS, and the WENO scheme ................42

Figure 3.12. The dissipation in WUCS, WCS, and the WENO scheme ................43

Figure 4.1 The numerical solution from WUCS when $\mathrm{u}_{0}(\mathrm{x})=\sin (\pi \mathrm{x})$

at different time steps.........................................................................53

Figure 4.2 The numerical solution from WUCS when $\mathrm{u}_{0}(\mathrm{x})=\cos (\pi \mathrm{x})$ at different time steps..........................................................................53

Figure 4.3. The numerical solution from WUCS when $\mathrm{u}_{0}(\mathrm{x})=\sin (3 \pi \mathrm{x})$ at different time steps..........................................................................53

Figure 4.4. The numerical solution from WUCS when $\mathrm{u}_{0}(\mathrm{x})=(\sin (\pi \mathrm{x}))^{2}$

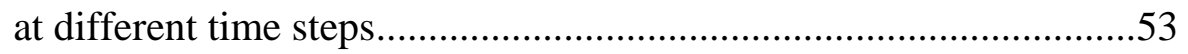

Figure 4.5 The exact solution compared with the numerical solutions from both WUCS and the WENO scheme with $\mathrm{N}=40$........................56

Figure 4.6 The enlarged portion of the selected part as indicated

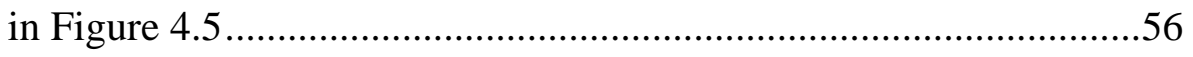

Figure 4.7 The exact solution compared with the numerical solution

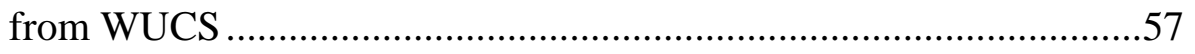

Figure 4.8 The error of the numerical solution from WUCS at tk=0.5

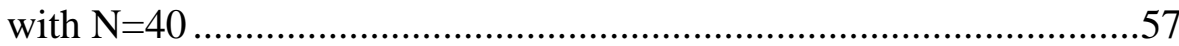

Figure 4.9 The numerical solution from WUCS when $\mathrm{u}_{0}(\mathrm{x})=\sin (\pi \mathrm{x})$ at different time steps......................................................................... 
Figure 4.10 The numerical solution from WUCS when $\mathrm{u}_{0}(\mathrm{x})=\sin (\pi \mathrm{x})$

at $t_{k}=0.5$.

Figure 4.11 The numerical solution from WUCS when

$\mathrm{u}(\mathrm{x}, 0)=\frac{1}{2}(1-\cos (\pi \mathrm{x}))$ at different time steps 58

Figure 4.12 The numerical solution from WUCS when

$\mathrm{u}(\mathrm{x}, 0)=\frac{1}{2}(1-\cos (\pi \mathrm{x}))$ at different time steps

Figure 4.13 The shock tube problem. Distribution of pressure at $t=2$. 61

Figure 4.14 The enlarged portion of the selected part as indicated

in Figure 4.13.

Figure 4.15 The shock tube problem. Distribution of density solution at $\mathrm{t}=2$ 61

Figure 4.16 The enlarged portion of the selected part as indicated in Figure 4.15 61

Figure 4.17 The shock tube problem. Distribution of velocity at $\mathrm{t}=2$ 62

Figure 4.18. The enlarged portion of the selected part as indicated in Figure 4.17 62

Figure 4.19. The shock tube problem. Distribution of energy at $t=2$ 62

Figure 4.20. The enlarged portion of the selected part as indicated in Figure 4.19 62

Figure 4.21 The shock tube problem. The enlarged portion of the selected part as indicated in Figure 4.13 63 
Figure 4.22 The shock tube problem. The enlarged portion of the

selected part as indicated in Figure 4.15

Figure 4.23 The shock tube problem. The enlarged portion of the

selected part as indicated in Figure 4.17

Figure 4.24 The shock tube problem. The enlarged portion of the selected part as indicated in Figure 4.19

Figure 4.25 The shock-entropy interaction problem. Distribution of pressure at $\mathrm{t}=1.8$ 64

Figure 4.26 The enlarged portion of the selected part as indicated in Figure 4.25 .64

Figure 4.27 The shock-entropy interaction problem. Distribution of density at $\mathrm{t}=1.8$ 65

Figure 4.28 The enlarged portion of the selected part as indicated in Figure 4.27 65

Figure 4.29 The shock-entropy interaction problem. Distribution of velocity at $\mathrm{t}=1.8$. .65

Figure 4.30 The enlarged portion of the selected part as indicated in Figure 4.29 .65

Figure 4.31 The shock-entropy interaction problem. Distribution of energy at $t=1.8$. .66

Figure 4.32 The enlarged portion of the selected part as indicated in Figure 4.31 66 
Figure 4.33 The shock-entropy interaction problem. The enlarged portion of the selected part as indicated in Figure 4.25

Figure 4.34 The shock-entropy interaction problem. The enlarged portion of the selected part as indicated in Figure 4.27 ................................67

Figure 4.35 The shock-entropy interaction problem. The enlarged portion of the selected part as indicated in Figure 4.29 .................................67

Figure 4.36 The shock-entropy interaction problem. The enlarged portion of the selected part as indicated in Figure 4.31 67 


\section{LIST OF TABLES}

Table 2.1 The $\mathrm{L}_{\infty}$ order for both SFSM and MBFM when $\mathrm{u}^{\prime \prime}=12 \mathrm{x}^{2} \ldots \ldots \ldots \ldots \ldots \ldots . . . . . .12$

Table 2.2 The $\mathrm{L}_{\infty}$ order for both SFSM and MBFM when $\mathrm{u}^{\prime \prime}=20 \mathrm{x}^{3} \ldots \ldots \ldots \ldots \ldots \ldots . . . . . . .13$

Table 2.3 The $\mathrm{L}_{\infty}$ order for both SFSM and MBFM when $\mathrm{u}^{\prime \prime}=\mathrm{e}^{\mathrm{x}} \ldots \ldots \ldots \ldots \ldots \ldots \ldots . . . . . . . . . .14$

Table 4.1 Errors of the numerical solution from WUCS

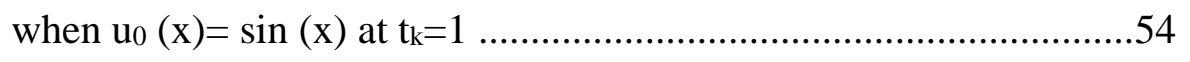

Table 4.2 Errors of the numerical solution from WUCS

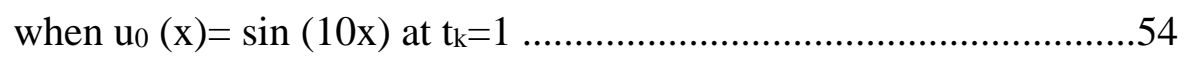

Table 4.3 Errors of the numerical solution from WUCS

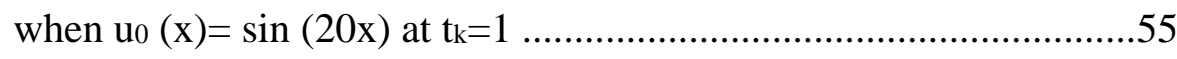

Table 4.4 Errors of the numerical solution from WUCS

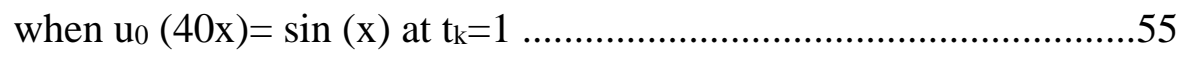

Table 4.5 Errors of the numerical solution from WUCS

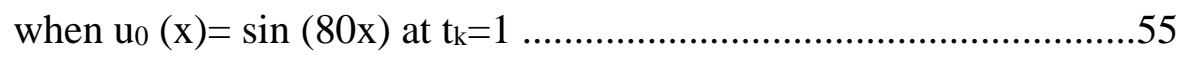

Table 4.6 Errors of the numerical solution from WUCS

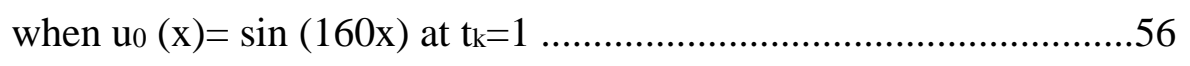

Table 4.7 Errors of the numerical solution obtained by WUCS of the

Burgers' equation when $\mathrm{u}_{0}(\mathrm{x})=\sin (\pi \mathrm{x}), 0<\mathrm{x}<1$ at $\mathrm{t}=1$ 


\section{CHAPTER 1}

\section{INTRODUCTION}

Due to an increase of computational resources, numerical schemes have been widely improved. One of the critical problems in numerical analysis is approximating the derivative numerically for a given data set. Many problems require a high order scheme with high resolution. Others that involve shocks require a numerical scheme with high resolution and shock capturing capability. Hence, constructing schemes with good spectral properties and shock capturing capacity is very desirable in computational fluid dynamics (CFD).

Spectral methods are very powerful and efficient tools that have been widely applied during the last decades to find the numerical solutions of partial differential equations. They have a wide range of applications due to their high accuracy compared to other methods, such as finite difference and finite element methods. One of these methods is the Fourier or pseudo-spectral method which has the advantages of the Fast Fourier Transform (FFT). FFT is simply an algorithm that performs the same process as the Discrete Fourier Transform (DFT) in a much faster way because FFT requires only $\mathrm{O}(\mathrm{N} \log \mathrm{N})$ arithmetical operations compared with DFT that takes $\mathrm{O}\left(\mathrm{N}^{2}\right)$ operations to obtain the same result. In addition, since the Fourier spectral method has high resolution with high order, it can be considered as an efficient analytical or numerical technique in which exact or approximate 
solutions can be calculated for many types of differential equations. Several phenomena in physics involve periodic directions, so the Fourier spectral method can be used efficiently to approximate the solutions of mathematical models that represent these phenomena.

As is well known, the standard Fourier spectral method can be only accurate when applied to periodic and smooth problems. It is stable and yields spectral convergence, but this is not the case when dealing with non-periodic or non-smooth problems because of the Gibbs phenomenon, which causes oscillations near the boundary or the discontinuous points. For that reason, people have tried to overcome this problem; many techniques and approaches have been attempted. One approach is multiplying the function by a smooth window function $w$, where $w$ and its derivatives are close to zero at the boundary points. Another approach is relying on the basis of Gegenbauer polynomials when using the truncated Fourier series of the function to reconstruct a non-periodic function and re-expand these series into that basis. Other people have different approaches by trying to filter out the oscillations. Moreover, the approach of Chebyshev polynomials is the most popular one which computes the Fourier series of the transformed function after using some periodic transformations.

All approaches described above, which attempt to overcome the nonperiodic or non-smooth situations, are successful; however, they still have side effects. For example, the computation cost will increase when using Chebyshev 
spectral methods because they require more points per wavelength to resolve the function compared to the Standard Fourier Spectral Method (SFSM). Furthermore, special polynomials with some restrictions are required to treat the points near the boundaries.

In this dissertation, the Modified Buffered Fourier Method (MBFM) is proposed to treat the weakness of SFSM by adding a 25\% buffer zone to make the function periodic on the boundaries as well as some of its derivatives in the extended domain. Therefore, this method can be applied for smooth and nonperiodic boundary conditions problems. For non-smooth partial differential equations, further research is needed so the method may be applied when a good shock detector is used to determine the shock location. Hence, finding a numerical scheme with shock capturing capacity leads to the main approach of this dissertation.

In recent years, many shock capturing schemes have been extensively developed and used in CFD. Before the ENO (Essentially Non-Oscillatory) scheme was introduced by Harten (1987), adding artificial viscosities and applying limiters were the most common ways to reduce or eliminate the oscillations near discontinuities. One problem of using the first approach is the fine adapting of the parameter controlling the size of the artificial viscosity, and it is problem dependent. In addition, reducing the order of accuracy is a serious problem of the second approach (Shu, 1997). Therefore, the ENO approach was identified as the 
first successful strategy that deals with problems containing both shocks and complicated smooth flow structures, like a turbulent flow and shock interaction with vortices (Shu, 1997). The main idea of the ENO scheme is to select the smoothest stencil among three stencils, and one of the disadvantages is the scheme has only second order because of using only three points. In addition, if the three candidates are almost the same, it is difficult to decide which one to use. To overcome the low order accuracy of the ENO scheme, Jiang and Shu (1996) proposed a weighted ENO scheme (WENO). In the WENO scheme, optimal weights are assigned to all stencils, which are combined to get a higher order in smooth areas and bias upwind weights to avoid the stencils containing shocks. The WENO scheme has been widely used, and it produces very successful results. However, it costs a little high because of the complexity of calculating the weights in each stencil at each iteration step.

Lele (1992) proposed a family of finite difference compact schemes (CS), which have a range of scales in the evaluation of low and high order derivatives. When using CS, the order of the scheme can be increased by involving both function and derivatives at the same given points while the desired approximation order in the standard finite difference scheme requires at least one point wider. Although CS can keep high order accuracy and high resolution in smooth regions, they cannot capture shocks because of the global dependence on the data set. Grid to grid oscillation will be introduced when using CS to differentiate functions with 
discontinues. Many techniques have been used along with CS, such as filtering, which still cannot eliminate oscillations near shock areas (Visbal et al., 1998; Shan et al., 1999; Jiang et al., 1999).

In this dissertation, two different approaches are used to get two kinds of numerical schemes. In the first scheme, all advantages of the FFT algorithm have been possessed, and the weakness has been treated by introducing the Modified Buffered Fourier Method (MBFM). MBFM uses the technics of a smooth buffered zone to make the function and some of its derivatives periodic in the extended domain. Secondly and most importantly, a high order scheme with shock capturing capacity is proposed. To increase the order, the idea of weighted compact scheme (WCS) is used. However, using the idea of compact scheme (CS) will lead to a global dependent property, which is prohibited in shock areas. Also, the left stencil of WCS has a negative dissipation. To avoid the global dependence near shock areas, the derivative matrix is decoupled to change the global dependence problem into an up-winding dependence. Meanwhile, to overcome the problem of WCS, an upwinding scheme has been constructed so the left and central candidates have a positive dissipation. Hence, the Weighted Upwinding Compact Scheme (WUCS) is introduced in this dissertation.

In chapter 2 of this dissertation, different forms of the Fourier transform are illustrated in section 2.1. The idea of MBFM and its steps are discussed in 
section 2.2. Section 2.3 has the numerical results calculated by MBFM and compared with SFSM. The conclusion of this chapter is introduced in section 2.4.

Chapter 3 of this dissertation is mainly about WUCS. In section 3.1, previous numerical schemes which are related to WUCS are introduced. Basic formulation and schemes of WUCS are discussed in section 3.2. In section 3.3 the dispersion and dissipation analysis of WUCS is introduced and compared to the related schemes. The idea of decoupling the system is illustrated in section 3.4. Also, chapter 3 is concluded in section 3.5.

WUCS is applied to many one-dimensional typical problems in chapter 4 . Finally, chapter 5 has the conclusion and future work of this dissertation. 


\section{CHAPTER 2}

\section{MODIFIED BUFFERED FOURIER METHOD}

\subsection{Fourier Transform}

\subsubsection{Continuous Fourier Transform}

For a function $\mathrm{f}(\mathrm{x})$ defined on a finite interval, $-\pi<\mathrm{x}<\pi$, the Fourier transform $\hat{\mathrm{f}}_{\mathrm{k}}$ is a set of discrete values defined on an infinite grid as follows:

$$
\hat{f}_{k}=\int_{-\pi}^{\pi} f(x) e^{-i k x} d x
$$

for $k=0, \pm 1, \pm 2, \ldots$.

The inverse Fourier transform is defined as

$$
f(x)=\frac{1}{2 \pi} \sum_{k=-\infty}^{\infty} \hat{f}_{k} e^{i k x}
$$

for $-\pi<x<\pi$

\subsubsection{Discrete Fourier Transform (DFT)}

For a discrete grid function $f_{j}$ on an infinite grid at points $x_{j}=j h$ and $k=0, \pm 1, \pm 2, .$. , the Fourier transform $\hat{f}(t)$ is a function defined on a finite interval $\left[\frac{-\pi}{h}, \frac{\pi}{h}\right]$ as

$$
\hat{f}(t)=\sum_{j=-\infty}^{\infty} f_{j} e^{-i t x_{j}}
$$

for $-\frac{\pi}{h}<t<\frac{\pi}{h}$ and $h>0$

The inverse Fourier transform is defined as

$$
f_{j}=\frac{1}{2 \pi} \int_{-\frac{\pi}{h}}^{\frac{\pi}{h}} \hat{f}(t) e^{i t x_{j}} d t
$$

for $\mathrm{j}=0, \pm 1, \pm 2, \ldots$. 
The Fourier transform can be written in different forms, and the following form is often used computationally. For a discrete grid function $f_{j}$ on a grid with $N=\frac{2 \pi}{h}$ points in the interval $[0,2 \pi]$, the grid points are $x_{j}=j h$ for $k=1,2,3, \ldots$ where $h=\frac{2 \pi}{N}$. The Fourier transform $\hat{\mathrm{f}}_{\mathrm{k}}$ is a set of $\mathrm{N}$ discrete values for wave numbers

$$
\mathrm{t}_{\mathrm{k}}=\mathrm{k}=\frac{-\mathrm{N}}{2}+1, \ldots, \frac{\mathrm{N}}{2}
$$

defined as

$$
\hat{\mathrm{f}}_{\mathrm{k}}=\mathrm{h} \sum_{\mathrm{j}=1}^{\mathrm{N}} \mathrm{f}_{\mathrm{j}} \mathrm{e}^{-\mathrm{ikx} \mathrm{x}_{\mathrm{j}}}
$$

for $\mathrm{k}=\frac{-\mathrm{N}}{2}+1, \ldots, \frac{\mathrm{N}}{2}$

The inverse Fourier transform is defined as

$$
f_{j}=\frac{1}{2 \pi} \sum_{k=\frac{-N}{2}+1}^{\frac{N}{2}} \hat{f}_{k} e^{i k x_{j}}
$$

for $\mathrm{k}=1,2,3, \ldots, \mathrm{N}$

For 2-D, the DFT is given by

$$
\hat{\mathrm{f}}_{\mathrm{m}, \mathrm{n}}=\sum_{\mathrm{j}=0}^{\mathrm{M}-1} \sum_{\mathrm{K}=0}^{\mathrm{N}-1} \mathrm{e}^{-2 \pi \mathrm{i}\left(\frac{\mathrm{mj}}{\mathrm{M}}+\frac{\mathrm{nk}}{\mathrm{N}}\right)} \mathrm{f}_{\mathrm{j}, \mathrm{k}}
$$

where $\mathrm{m}=0,1, \ldots, \mathrm{N}-1$ and $\mathrm{n}=0,1, \ldots, \mathrm{N}-1$

and the DFT inverse is defined as

$$
\mathrm{f}_{\mathrm{j}, \mathrm{k}}=\frac{1}{\mathrm{MN}} \sum_{\mathrm{m}=0}^{\mathrm{M}-1} \sum_{\mathrm{n}=0}^{\mathrm{N}-1} \mathrm{e}^{2 \pi \mathrm{i}\left(\frac{\mathrm{mj}}{\mathrm{M}}+\frac{\mathrm{nk}}{\mathrm{N}}\right)} \hat{\mathrm{f}}_{\mathrm{m}, \mathrm{n}}
$$

where $\mathrm{j}=0,1, \ldots, \mathrm{N}-1$ and $\mathrm{k}=0,1, \ldots, \mathrm{N}-1$ 


\subsubsection{Standard Fourier Spectral Method (SFSM)}

In this method, finding the derivative of a function $f$ is based on the properties of the frequency domain or the Fourier domain. From (2.6), each point of the original function $\mathrm{f}$ can be approximated as

$$
\mathrm{f}_{\mathrm{j}}=\frac{1}{\mathrm{~N}} \sum_{\mathrm{k}=0}^{\mathrm{N}-1} \hat{\mathrm{f}}_{\mathrm{k}} \mathrm{e}^{\mathrm{ikx} \mathrm{x}_{\mathrm{j}}} \text { for } \mathrm{j}=0,1, \ldots, \mathrm{N}-1
$$

Taking the derivative of both sides of (2.9) results in

$$
\left(\mathrm{f}_{\mathrm{j}}\right)^{\prime}=\frac{1}{\mathrm{~N}} \sum_{\mathrm{k}=0}^{\mathrm{N}-1} \hat{\mathrm{f}}_{\mathrm{k}} \mathrm{e}^{\mathrm{ikx_{j }}}(\mathrm{ik}) \text { for } \mathrm{j}=0,1, \ldots, \mathrm{N}-1
$$

where $\hat{\mathrm{f}}_{\mathrm{k}}=\sum_{\mathrm{j}=0}^{\mathrm{N}-1} \mathrm{f}_{\mathrm{j}} \mathrm{e}^{-\mathrm{ikx} \mathrm{j}_{\mathrm{j}}} \quad$ for $\mathrm{k}=0,1, \ldots, \mathrm{N}-1$

and $\left(\mathrm{f}_{\mathrm{j}}\right)^{\prime}$ is the derivative of the original function $\mathrm{f}$ at the point $x_{\mathrm{j}}$.

The following notation illustrates the relation between the function $\mathrm{f}$ and its Fourier expansion:

$$
\begin{aligned}
\mathrm{f}(\mathrm{t}) & \Leftrightarrow \hat{\mathrm{f}}(k) \\
\frac{d \mathrm{f}(\mathrm{t})}{d t} & \Leftrightarrow i k \hat{\mathrm{f}}(k)
\end{aligned}
$$

\subsection{Modified Buffered Fourier Method (MBFM)}

For smooth and periodic functions, the Fast Fourier Transform (FFT) algorithm can be applied successfully, but it is not suitable for non-periodic functions. To overcome this issue, a Modified Buffered Fourier Method (MBFM) is illustrated in this chapter. The proposed method can deal with non-periodic problems by using the idea of the buffered zone, which can be implemented by extending the domain and adding smooth 
interpolations. In the Standard Fourier Spectral Method (SFSM), the derivative of the source function might not be periodic on the boundaries, so large oscillation near the boundaries may appear. However, the oscillation will be highly reduced when using MBFM because MBFM can make the function and its derivative periodic on the boundaries.

As shown in (2.12), the derivative at a point $t$ of the function $\mathrm{f}$ can be easily calculated by multiplying by its corresponding number ik. However, dividing by a corresponding number $i k$ of the point $t$ of the function $f$ gives only the integration of the function $f(t)+c$ for only a zero constant $c$. Therefore, to recover the nonzero constants in the time domain, a delta function $\delta(k)$ should be added in the frequency domain as follows:

$$
\int_{-\infty}^{t} f(x) d x \Leftrightarrow \frac{\hat{\mathrm{f}}(k)}{i k}+\pi \hat{\mathrm{f}}(0) \delta(k)
$$

As mentioned in section (2.1.3), SFSM requires periodicity and smoothness, and MBFM proposes a simple way to make the function periodic and smooth by implementing the three steps described below.

\subsubsection{Transformation}

In this step, if the source function $f$ is not periodic at the boundaries, a linear function $g$ should be subtracted from the function $f$ so that $f-g$ is periodic in its values on the boundaries. The linear function g has the following formula

$$
g(x)=\frac{f(b)-f(a)}{b-a} x
$$




\subsubsection{Buffer zone extension}

Next, the $f-g$ function from the previous step can be extended by using a smooth polynomial, which can be determined by 8 points, 4 from the left and 4 from the right. Also, the smooth polynomial is constructed according to the cubic spline interpolation.

\subsubsection{Normalization}

Finally, the key step of MBFM is to make the zero Fourier coefficient of the extended function, say a function $w$, zero, so (2.13) can be used to find the integration. Therefore, a constant $c$ will be subtracted from this extended function w so that $(\widehat{w-c})(0)=0$.

\subsection{Numerical Results}

In this section, MBFM is applied to several examples to illustrate the efficiency of the suggested method. The results from this method are also compared with the SFSM. It can be clearly seen from tables 2.1-2.3 that the order of accuracy is highly improved from two for SFSM to almost seven for MBFM.

2.3.1. $u^{\prime \prime}=12 x^{2}, x \in[-1,1], u(-1)=u(1)=1$

As needed, the derivative of the source function should be periodic on the boundaries, so before normalizing the source function, a buffer polynomial should be constructed using cubic spline interpolation (figure 2.1). Also, a buffer zone should be added to the source function to form an extended function (figure 2.2). 


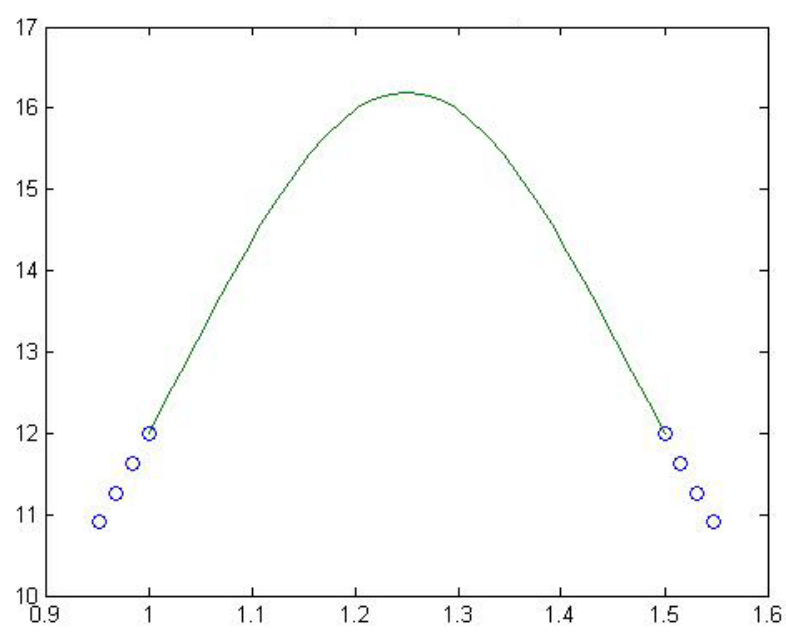

Figure 2.1. The buffer polynomial of 8 points constructed according to the cubic spline interpolation.

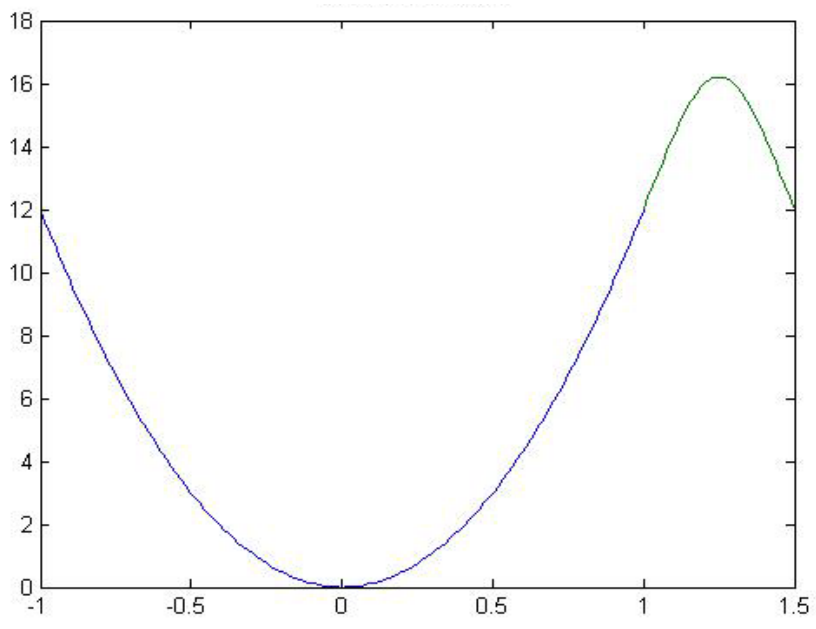

Figure 2.2 The extended function at the new domain.

Table 2.1 shows that the $\mathrm{L}_{\infty}$ errors of the numerical results obtained from MBFM are much smaller compared with the errors from SFSM.

Table 2.1. The $\mathrm{L}_{\infty}$ order for both SFSM and MBFM when $u^{\prime \prime}=12 x^{2}$

\begin{tabular}{|c|c|c|c|c|c|c|}
\hline \multirow{2}{*}{$\mathbf{N}$} & \multicolumn{3}{|c|}{ SFSM } & \multicolumn{3}{|c|}{ MBFM } \\
\hline & $\mathbf{L}_{\infty}$ error & $h / 2 h$ & $\mathbf{L}_{\infty}$ order & $\mathbf{L}_{\infty}$ error & $\mathbf{h} / \mathbf{2 h}$ & $\mathbf{L}_{\infty}$ order \\
\hline 64 & $1.36 \mathrm{E}-05$ & & & 2.39E-05 & & \\
\hline 128 & $1.11 \mathrm{E}-07$ & $1.23 \mathrm{E}+02$ & 6.94 & $1.97 \mathrm{E}-07$ & $1.22 \mathrm{E}+02$ & 6.93 \\
\hline 256 & $8.78 \mathrm{E}-10$ & $1.26 \mathrm{E}+02$ & 6.98 & 1.56E-09 & $1.26 \mathrm{E}+02$ & 6.98 \\
\hline 512 & 6.89E-12 & $1.28 \mathrm{E}+02$ & 6.99 & $1.22 \mathrm{E}-11$ & $1.28 \mathrm{E}+02$ & 7.00 \\
\hline
\end{tabular}


2.3.2. $u^{\prime \prime}=20 x^{3}, x \in[0,1], u(0)=0, u(1)=1$

After the source function is transformed, we construct the buffer zone and normalize the extended source as shown in figures 2.3 and 2.4. When applying SFSM to the extended domain and deleting the buffer, the numerical solution can be obtained.

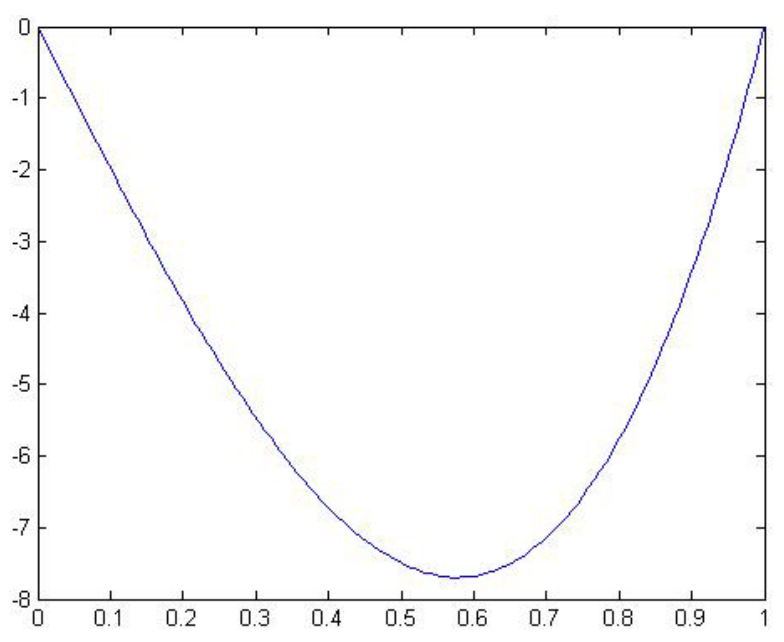

Figure 2.3. The source function after transformation.

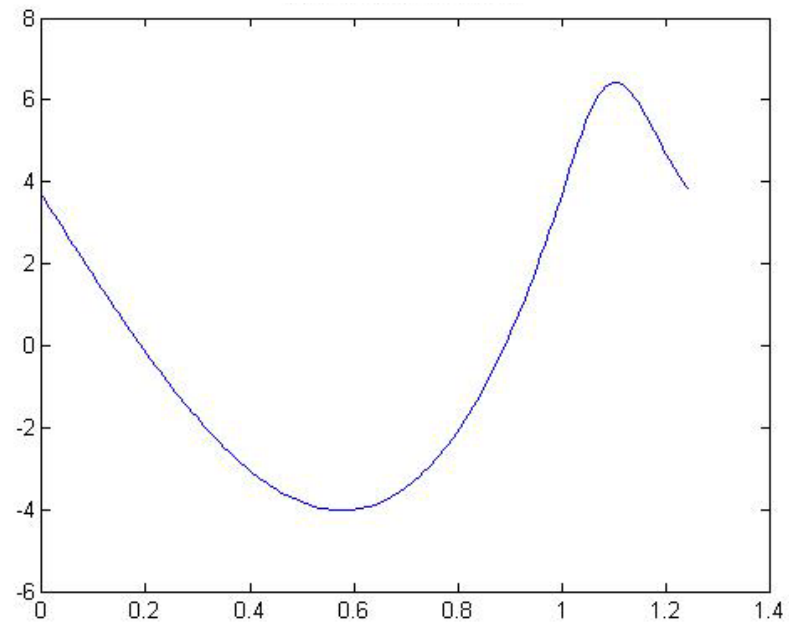

Figure 2.4. The shifted buffered function at the new domain.

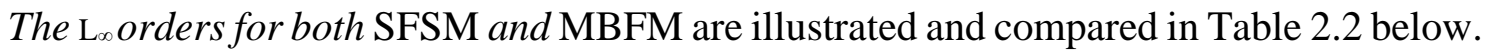

Table 2.2. The $\mathrm{L}_{\infty}$ order for both SFSM and MBFM when $u^{\prime \prime}=20 x^{3}$

\begin{tabular}{||c|ccc|ccc||}
\hline \hline \multirow{2}{*}{$\mathbf{N}$} & \multicolumn{3}{|c|}{ SFSM } & \multicolumn{3}{c||}{ MBFM } \\
\cline { 2 - 7 } & $\mathbf{L}_{\infty}$ error & $\mathbf{h} / \mathbf{2 h}$ & $\mathbf{L}_{\infty}$ order & $\mathbf{L}_{\infty}$ error & $\mathbf{h} / \mathbf{2 h}$ & $\mathbf{L}_{\infty}$ order \\
\hline 64 & $1.51 \mathrm{E}-04$ & & & $4.68 \mathrm{E}-10$ & & \\
128 & $3.79 \mathrm{E}-05$ & $3.98 \mathrm{E}+00$ & $\mathbf{1 . 9 9}$ & $5.30 \mathrm{E}-12$ & $8.82 \mathrm{E}+01$ & $\mathbf{6 . 4 6}$ \\
256 & $9.51 \mathrm{E}-06$ & $3.99 \mathrm{E}+00$ & $\mathbf{1 . 9 7}$ & $5.10 \mathrm{E}-14$ & $1.04 \mathrm{E}+02$ & $\mathbf{6 . 7 0}$ \\
512 & $2.38 \mathrm{E}-06$ & $3.99 \mathrm{E}+00$ & $\mathbf{2 . 0 0}$ & $4.40 \mathrm{E}-16$ & $1.16 \mathrm{E}+02$ & $\mathbf{6 . 8 5}$ \\
\hline \hline
\end{tabular}


2.3.3. $u^{\prime \prime}=e^{x}, x \in[-1,1], u(-1)=e^{-1}, u(1)=e$

MBFM can be used to solve ODEs for any source function on any interval. The transformed source function and the shifted buffered function are shown in figures 2.5 and 2.6, respectively.

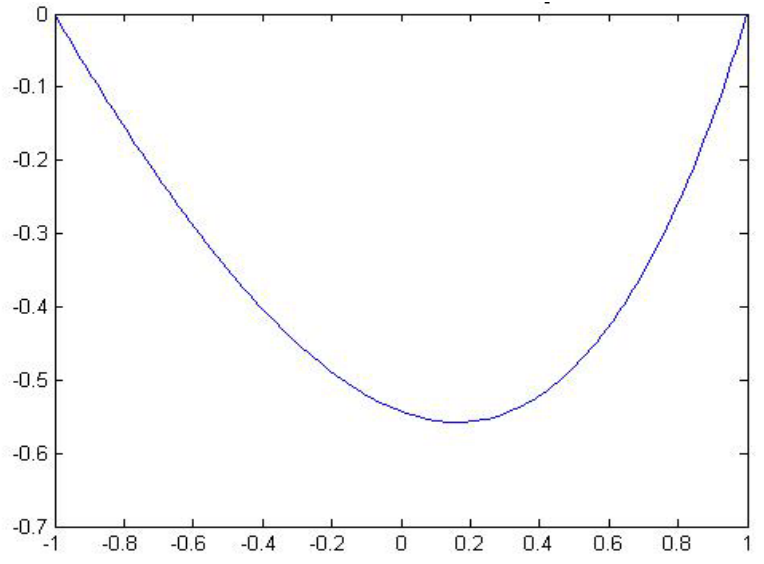

Figure 2.5. The transformed source function.

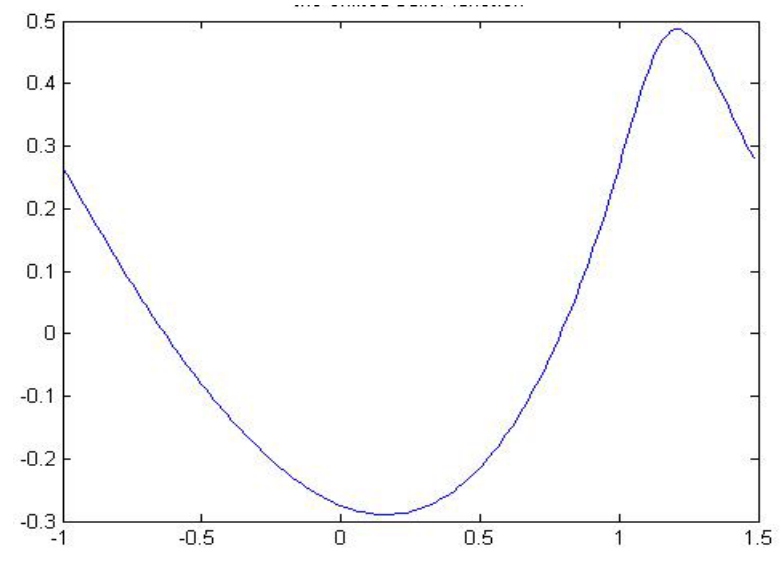

Figure 2.6. The shifted buffered function at the new domain.

Table 2.3 below compares the errors for both SFSM and MBFM, and it illustrates that MBFM can improve the order of accuracy and keep the same resolution.

Table 2.3. The $\mathrm{L}_{\infty}$ order for both SFSM and MBFM when $u^{\prime \prime}=e^{x}$

\begin{tabular}{||c|ccc|ccc||}
\hline \multirow{2}{*}{$\mathbf{N}$} & \multicolumn{3}{|c|}{ SFSM } & \multicolumn{3}{c||}{ MBFM } \\
\cline { 2 - 7 } & $\mathbf{L}_{\infty}$ error & $\mathbf{h} / \mathbf{2 h}$ & $\mathbf{L}_{\infty}$ order & $\mathbf{L}_{\infty}$ error & $\mathbf{~ h / 2 h}$ & $\mathbf{L}_{\infty}$ order \\
\hline 64 & $4.73 \mathrm{E}-05$ & & & $1.47 \mathrm{E}-10$ & & \\
128 & $1.19 \mathrm{E}-05$ & $3.98 \mathrm{E}+00$ & $\mathbf{1 . 9 9}$ & $1.66 \mathrm{E}-12$ & $8.85 \mathrm{E}+01$ & $\mathbf{6 . 4 7}$ \\
256 & $2.98 \mathrm{E}-06$ & $3.99 \mathrm{E}+00$ & $\mathbf{2 . 0 0}$ & $1.59 \mathrm{E}-14$ & $1.04 \mathrm{E}+02$ & $\mathbf{6 . 7 0}$ \\
512 & $7.46 \mathrm{E}-07$ & $3.99 \mathrm{E}+00$ & $\mathbf{2 . 0 0}$ & $1.38 \mathrm{E}-16$ & $1.15 \mathrm{E}+02$ & $\mathbf{6 . 8 5}$ \\
\hline
\end{tabular}




\subsection{Conclusion}

All advantages of the FFT algorithm are possessed, and the weaknesses are treated in this chapter by introducing the Modified Buffered Fourier Method (MBFM), which uses the technics of a smooth buffered zone. As shown from the numerical results, MBFM can obtain very accurate numerical derivatives for non-periodic functions. Large errors only happen near the boundaries because of the non-periodicity of the function and the polynomial interpolation. Also, the Modified Buffered Fourier Method (MBFM) keeps high resolution and high order accuracy for smooth PDEs, and the order of accuracy is effected by the interpolation on the boundaries. Hence, some problems from the simulation of transitional and turbulent flows might be solved by the proposed method. For non-smooth PDEs, the method may be applied when a good shock detector is used to determine the shock location. 


\section{CHAPTER 3}

\section{WEIGHTED UPWINDING COMPACT SCHEME (WUCS)}

\subsection{Numerical Formula}

A Weighted Upwinding Compact Scheme (WUCS) uses the idea of Weighted Compact Scheme (WCS) to increase the order at each stencil. Also, WUCS applies the bias weight from the WENO scheme at each stencil according to the smoothness of each one. In the present section, a brief description for each scheme that related to WUCS is provided.

\subsubsection{Essentially Non-Oscillatory (ENO) scheme and conservative reconstruction}

In 1987, Harten, Osher, Engquist, and Chakravarthy introduced the ENO scheme in the form of cell averages. Among several stencils, the ENO scheme chooses the smoothest one to approximate the fluxes at cell boundaries to increase the order and to reduce oscillations near the boundaries. The conservative property of the scheme is essential, especially when dealing with problems that have discontinuities because large errors will be generated near discontinuities when using neoconservative methods. One problem with the finite difference compact schemes is the conservative property of the schemes. The cell-averaged version of the ENO scheme is costly and complicated for multi-dimensional problems because of the procedure of reconstructing point values from cell averages. Hence, to avoid this reconstruction procedure, the flux version of the ENO scheme was introduced by Shu (1988) and Osher (1989). In this work, the ENO 
reconstruction method is used together with a weighted compact scheme to obtain conservation as described below (Shu \& Osher, 1988, 1989). For 1-D conservation laws,

$$
u_{t}(x, t)+f_{x}(u(x, t))=0
$$

After discretizing the domain, the cell interface (grid), the cell center, and the cell size are defined as follows, respectively.

$$
\begin{gathered}
a=x_{1 / 2}<x_{3 / 2}<x_{5 / 2}<\cdots<x_{N-\frac{1}{2}}<x_{N+\frac{1}{2}}=b \\
x_{j}=\frac{1}{2}\left(x_{j-\frac{1}{2}}+x_{j+\frac{1}{2}}\right) \\
h=x_{j+\frac{1}{2}}-x_{j-\frac{1}{2}}, j=1,2, \ldots, N
\end{gathered}
$$

Figure 3.1 below illustrates the grid above where the triangles denote the cell interfaces (3.2), and the circles denote the cell centers (3.3).

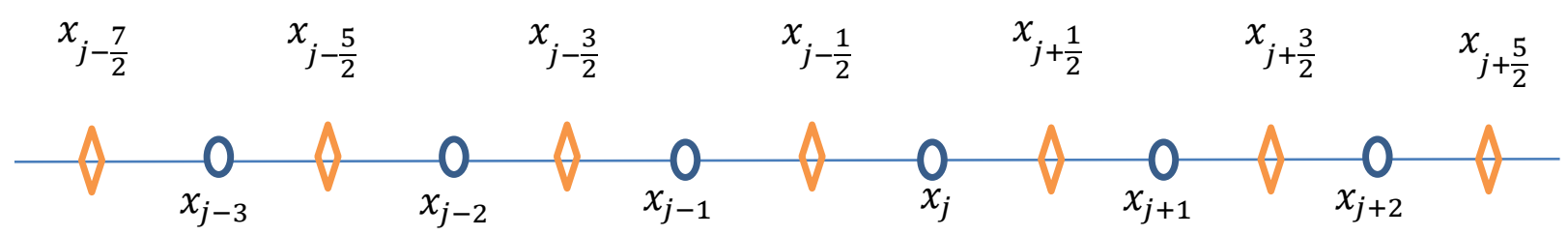

Figure 3.1. Grid for 1-D case.

A semi-discrete conservative form of (3.1) can be described when a conservative approximation to the spatial derivative is applied as follows:

$$
\frac{d u_{j}}{d t}=\frac{-\left(\hat{f}_{j+\frac{1}{2}}-\hat{f}_{j-\frac{1}{2}}\right)}{h}
$$

where $\hat{f}$ is the numerical flux, 


$$
f_{j}=f\left(u\left(x_{j}, t\right)\right)=\frac{\int_{x-\frac{1}{2}}^{x}{ }_{j+\frac{1}{2}}^{f}(\xi) d \xi}{h},
$$

$h$ is the step size in the $\mathrm{x}$-direction, and $H$ is the primitive function of $\hat{f}$ defined as

$$
\begin{gathered}
H_{j+\frac{1}{2}}=H\left(x_{j+\frac{1}{2}}\right)=\int_{-\infty}^{x_{j+\frac{1}{2}}} \hat{f}(\xi) d \xi=\sum_{i=-\infty}^{j} \int_{x_{i-\frac{1}{2}}}^{x} i+\frac{1}{2} \hat{f}(\xi) d \xi=\sum_{i=-\infty}^{j} f_{i} h \\
f_{j}=\frac{{ }_{\left.j+\frac{1}{2}-H_{j-\frac{1}{2}}\right)}}{h} \\
H^{\prime}{ }_{j+\frac{1}{2}}=\hat{f}_{j+\frac{1}{2}}, H^{\prime}{ }_{j-\frac{1}{2}}=\hat{f}_{j-\frac{1}{2}} \\
f^{\prime}\left(x_{j}\right)=f_{j}^{\prime}=\frac{\left(\hat{f}_{j+\frac{1}{2}}-\hat{f}_{j-\frac{1}{2}}\right)}{h}=\frac{\left(H^{\prime}{ }_{j+\frac{1}{2}}-H^{\prime}{ }_{j-\frac{1}{2}}\right)}{h}
\end{gathered}
$$

The described procedure above can be illustrated as follows:

$$
f \rightarrow H \rightarrow H^{\prime}=\hat{f} \rightarrow f^{\prime}
$$

Indeed, the only approximation involved is the calculation of the derivative of the primitive function $H$. The ENO scheme is successfully applied to many numerical experiments, but it has some drawbacks. One problem with the ENO scheme is the high cost of heavy usage of logical statements. Another problem is the free adaptive stencil, which is not required for smooth regions.

\subsection{2. $5^{\text {th }}$ order Weighted Essentially Non-Oscillatory (WENO) scheme}

To keep the advantages of the ENO scheme and overcome its drawbacks, Liu, Osher, and Chan proposed the Weighted Essentially Non-Oscillatory (WENO) scheme. The main idea of the WENO scheme is to assign optimal weights for all stencils to get a high order 
scheme in smooth areas and to assign bias weights near discontinuities to avoid the stencil containing a shock.

\subsubsection{Flux approximation}

In order to get the derivative of the given function $f$ at the point $x(j)$, first the approximation of $H^{\prime}{ }_{j+\frac{1}{2}}=\hat{f}_{j+\frac{1}{2}}$ is needed. Three candidates are given from the three stencils,

$$
S^{0}=\left\{F_{j-2}, F_{j-1}, F_{j}\right\}, S^{1}=\left\{F_{j-1}, F_{j}, F_{j+1}\right\} \text {, and } S^{2}=\left\{F_{j}, F_{j+1}, F_{j+2}\right\}
$$

as shown in figure 3.2 .

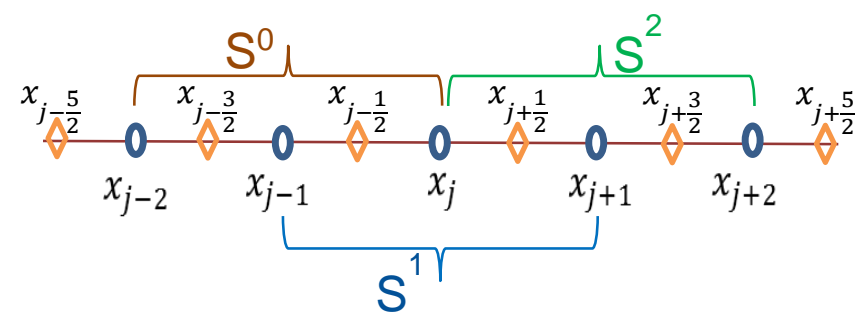

Figure 3.2. The stencils for the WENO scheme.

Each candidate is a third order polynomial, which can be found as follows.

For the left stencil $S^{0}=\left\{F_{j-2}, F_{j-1}, F_{j}\right\}$, let

$$
H^{\prime}{ }_{j+\frac{1}{2}}=\propto F_{j-2}+\beta F_{j-1}+\gamma F_{j}
$$

Substituting (3.7) in (3.11) results in

$$
\begin{gathered}
H_{j+\frac{1}{2}}^{\prime}=\frac{\propto\left(H_{j-2+\frac{1}{2}}-H_{j-2-\frac{1}{2}}\right)+\beta\left(H_{j-1+\frac{1}{2}}-H_{j-1-\frac{1}{2}}\right)+\gamma\left(H_{j+\frac{1}{2}}-H_{j-\frac{1}{2}}\right)}{h} \\
h H^{\prime}{ }_{j+1}=-\alpha H_{j-2}+(\propto-\beta) H_{j-1}+(\beta-\gamma) H_{j}+\gamma H_{j+1}
\end{gathered}
$$


Letting $\mathrm{h}=1, \mathrm{j}=0$, and $H=1, x, x^{2}, x^{3}$ gives the following system of equations:

$$
\begin{gathered}
1=\propto+\beta+\gamma \\
2=-3 \propto-\beta+\gamma \\
3=7 \propto+\beta+\gamma
\end{gathered}
$$

The solution of the above system is $\quad \alpha=\frac{1}{3}, \beta=-\frac{7}{6}$, and $\gamma=\frac{11}{6}$

Hence, $\quad S^{0}: \quad H_{j+\frac{1}{2}}^{\left(S^{0}\right)}=\frac{1}{3} F_{j-2}-\frac{7}{6} F_{j-1}+\frac{11}{6} F_{j}$

Similarly,

$$
\begin{gathered}
S^{1}:{H^{\prime}}_{j+\frac{1}{2}}^{\left(S^{1}\right)}=-\frac{1}{6} F_{j-1}+\frac{5}{6} F_{j}+\frac{1}{3} F_{j+1} \\
S^{2}:{H^{\prime}}_{j+\frac{1}{2}}^{\left(S^{2}\right)}=\frac{1}{3} F_{j}+\frac{5}{6} F_{j+1}-\frac{1}{6} F_{j+2}
\end{gathered}
$$

\subsubsection{Optimal weights for a high order accuracy}

A weighted average of the three stencils is considered with constant weights $c^{0}, c^{1}$, and $c^{2}$, such that

$$
S=c^{0} S^{0}+c^{1} S^{1}+c^{2} S^{2}
$$

For consistency of the scheme, (3.16) below should be satisfied

$$
\sum_{i=0}^{2} c^{\mathrm{i}}=1
$$

Substituting (3.12), (3.13), and (3.14) in (3.15) implies that

$$
\begin{gathered}
c^{0} H_{j+\frac{1}{2}}^{\prime}+c^{1} H^{\prime}{ }_{j+\frac{1}{2}}+c^{2} H^{\prime}{ }_{j+\frac{1}{2}}=c^{0}\left(\frac{1}{3} F_{j-2}-\frac{7}{6} F_{j-1}+\frac{11}{6} F_{j}\right)+c^{1}\left(-\frac{1}{6} F_{j-1}+\frac{5}{6} F_{j}\right. \\
\left.+\frac{1}{3} F_{j+1}\right)+c^{2}\left(\frac{1}{3} F_{j}+\frac{5}{6} F_{j+1}-\frac{1}{6} F_{j+2}\right)
\end{gathered}
$$




$$
H_{j+\frac{1}{2}}^{\prime}=c^{0}\left(\frac{1}{3} F_{j-2}-\frac{7}{6} F_{j-1}+\frac{11}{6} F_{j}\right)+c^{1}\left(-\frac{1}{6} F_{j-1}+\frac{5}{6} F_{j}+\frac{1}{3} F_{j+1}\right)+c^{2}\left(\frac{1}{3} F_{j}+\frac{5}{6} F_{j+1}-\frac{1}{6} F_{j+2}\right)
$$

From (3.16), we have

$$
c^{0}+c^{1}+c^{2}=1
$$

The two other equations can be found by letting $\mathrm{h}=1, \mathrm{j}=0$, and $H=x^{4}, x^{5}$ in (3.17).

Finally, when solving a 3x3 system, we have

$$
c^{0}=\frac{1}{10}, c^{1}=\frac{6}{10}, c^{2}=\frac{3}{10}
$$

Now, substituting (3.18) in (3.17) gives

$H^{\prime}{ }_{j+\frac{1}{2}}=\sum_{i=0}^{2} c^{\mathrm{i}}{H^{\prime}}_{j+\frac{1}{2}}^{\left(S^{\mathrm{i}}\right)}=\frac{1}{30} F_{j-2}-\frac{13}{60} F_{j-1}+\frac{47}{60} F_{j}+\frac{9}{20} F_{j+1}-\frac{1}{20} F_{j+2}$

Applying (3.19) in (3.9) leads to

$$
F_{j}^{\prime}=\frac{\left(-\frac{1}{30} F_{j-3}+\frac{1}{4} F_{j-2}-F_{j-1}+\frac{1}{3} F_{j}+\frac{1}{2} F_{j+1}-\frac{1}{20} F_{j+2}\right)}{h}
$$

Using the Taylor expansion for $F_{j-\mathrm{k}}$ results in

$$
F_{j}^{\prime}-\frac{\hat{F}_{j+\frac{1}{2}}-\hat{F}_{j-\frac{1}{2}}}{h}=\frac{1}{60} h^{5} F_{j}^{(6)}-\frac{1}{140} h^{6} F_{j}^{(7)}+\frac{1}{240} h^{7} F_{j}^{(8)}+\cdots
$$

(3.21) shows that the scheme with optimal weights and 6 grid points has a $5^{\text {th }}$ order truncation error, and it is the standard $5^{\text {th }}$ order bias upwind finite difference scheme.

\subsubsection{Non-linear weights for shock capturing}

The use of the constant weights $c^{0}, c^{1}$, and $c^{2}$ does not allow for an adaptive scheme according to the "smoothness" of the stencils. The non-linear weights of the WENO scheme adaptively chose each stencil due to its smoothness, and they are defined as follows: 


$$
\omega_{j \pm \frac{1}{2}}^{i}=\frac{\alpha_{j \pm \frac{1}{2}}^{i}}{\sum_{k=0}^{2} \alpha_{j \pm \frac{1}{2}}^{k}}, \propto_{j \pm \frac{1}{2}}^{i}=\frac{c^{i}}{(\underbrace{}_{j \pm \frac{1}{2}})^{i}}, \quad i=0,1,2
$$

where $\varepsilon$ is a small quantity to avoid the division by zero, and $\mathrm{p}$ is an integer number.

For consistency of the scheme, we must satisfy $\sum_{i=0}^{2} \omega_{j \pm \frac{1}{2}}^{i}=1$.

$I S_{j \pm \frac{1}{2}}^{i}$ is a "smoothness" indicator calculated by

$$
\begin{aligned}
& I S_{j \pm \frac{1}{2}}^{0}=\frac{13}{12}\left(F_{j-2}-2 F_{j-1}+F_{j}\right)^{2}+\frac{1}{4}\left(F_{j-2}-4 F_{j-1}+3 F_{j}\right)^{2} \\
& I S_{j \pm \frac{1}{2}}^{1}=\frac{13}{12}\left(F_{j-1}-2 F_{j}+F_{j+1}\right)^{2}+\frac{1}{4}\left(F_{j-1}-F_{j+1}\right)^{2} \\
& I S_{j \pm \frac{1}{2}}^{2}=\frac{13}{12}\left(F_{j}-2 F_{j+1}+F_{j+2}\right)^{2}+\frac{1}{4}\left(F_{j+2}-4 F_{j+1}+3 F_{j}\right)^{2}
\end{aligned}
$$

Finally, we have

$$
\widehat{F}_{j \pm \frac{1}{2}}=H_{j \pm \frac{1}{2}}^{\prime}=\sum_{i=0}^{2} \omega_{j \pm \frac{1}{2}}^{i} H^{\prime \prime}{ }_{j \pm \frac{1}{2}}^{\left(s^{i}\right)}
$$

\subsubsection{Boundary points schemes}

The following schemes are special $3^{\text {rd }}$ order schemes for the boundary points:

Point 0,

$$
\widehat{F}_{j-\frac{1}{2}}=\frac{11}{6} F_{j-1}-\frac{7}{6} F_{j}+\frac{1}{3} F_{j+1}
$$

Point 1,

$$
\widehat{F}_{j-\frac{1}{2}}=\frac{1}{3} F_{j}+\frac{5}{6} F_{j+1}-\frac{1}{6} F_{j+2}
$$

Point 2,

$$
\widehat{F}_{j-\frac{1}{2}}=-\frac{1}{6} F_{j-2}+\frac{5}{6} F_{j-1}+\frac{1}{3} F_{j}
$$

Point N-1,

$$
\widehat{F}_{j-\frac{1}{2}}=-\frac{1}{6} F_{j-1}+\frac{5}{6} F_{j}+\frac{1}{3} F_{j+1}
$$

Point N,

$$
\widehat{F}_{j-\frac{1}{2}}=\frac{1}{3} F_{j-2}-\frac{7}{6} F_{j-1}+\frac{11}{6} F_{j}
$$


The WENO scheme is a successful scheme by many users. However, some researchers in the DNS/LES community consider the scheme too dissipative for flow transition and turbulence because it has a $5^{\text {th }}$ order dissipation everywhere, and it is a $3^{\text {rd }}$ order dissipation near the shock. Also, the cost associated with the WENO scheme is a little high because of the complexity of calculating the weights in each stencil at each iteration step.

\subsubsection{The finite difference Compact Scheme (CS)}

With a uniform grid (Figure 3.3.), consider $x_{j}=h(j-1), f_{j}=f\left(x_{j}\right)$, and $f_{j}^{\prime}$ as the independent variable, the given function value, and the finite difference approximation to the first derivative of the function $\mathrm{f}$ at the point $\mathrm{j}$ for $1 \leq j \leq N$ respectively.

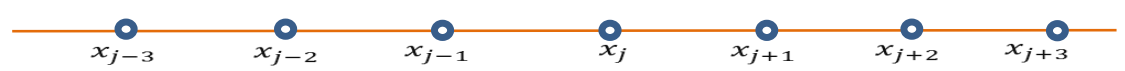

Figure 3.3. 1-D grid for the compact scheme.

The main idea of the compact schemes is to increase the order of the scheme by involving the function and its derivatives at the same given points. In the non-symmetric finite difference compact scheme (CS) (Lele, 1992), $f_{j}^{\prime}$ can be computed using the following formula:

$$
\beta_{-} f_{j-2}^{\prime}+\alpha_{-} f_{j-1}^{\prime}+f_{j}^{\prime}+\alpha_{+} f_{j+1}^{\prime}+\beta_{+} f_{j+2}^{\prime}=\frac{c_{-} F_{j-3}+b_{-} F_{j-2}+a_{-} F_{j-1}+a_{+} F_{j+1}+b_{+} F_{j+2}+c_{+} F_{j+3}}{h}
$$

The relations between the coefficients $\alpha_{+}, \alpha_{-}, \beta_{+}, \beta_{-}, a_{+}, a_{-}, b_{+}, b_{-}, c_{+}$, and $c_{-}$are derived by matching the Taylor series coefficients of various orders or using $f(x)=1, x, x^{2}$, 
$\ldots, \mathrm{x}^{\mathrm{k}}$. The first unmatched coefficients determine the formal truncation error of the approximation above. For the symmetric CS, the scheme can be constructed using the formula below:

$\beta F^{\prime}{ }_{j-2}+\alpha F^{\prime}{ }_{j-1}+F^{\prime}{ }_{j}+\alpha F^{\prime}{ }_{j+1}+\beta F^{\prime}{ }_{j+2}=c \frac{F_{j+3}-F_{j-3}}{6 h}+b \frac{F_{j+2}-F_{j-2}}{4 h}+a \frac{F_{j+1}-F_{j-1}}{2 h}$

Letting $\mathrm{h}=1, \mathrm{j}=0$, and $F=1, x$ in (3.26) gives a $2^{\text {nd }}$ order approximation as follows:

$$
\beta * 1+\alpha * 1+{F^{\prime}}^{\prime}+\alpha * 1+\beta * 1=c \frac{3 h-(-3 h)}{6 h}+b \frac{2 h-(-2 h)}{4 h}+a \frac{h-(-h)}{2 h}
$$

Hence, $\quad a+b+c=1+2 \alpha+2 \beta$

Similarly, $a+2^{2} b+3^{2} c=2 \frac{3 !}{2 !}\left(\alpha+2^{2} \beta\right) \quad\left(4^{\text {th }}\right.$ order $)$

$a+2^{4} b+3^{4} c=2 \frac{5 !}{4 !}\left(\alpha+2^{4} \beta\right) \quad$ (6 $6^{\text {th }}$ order $)$

$a+2{ }^{6} b+3^{6} c=2 \frac{7 !}{6 !}\left(\alpha+2^{6} \beta\right) \quad\left(8^{\text {th }}\right.$ order $)$

$a+2^{8} b+3^{8} c=2 \frac{9 !}{8}\left(\alpha+2^{8} \beta\right) \quad\left(10^{\text {th }}\right.$ order $)$

Although the idea of CS can increase the order of the scheme without increasing the number of the grid points, it cannot capture the shocks because of the global dependence on the data set.

\subsubsection{Weighted Compact Scheme (WCS)}

WCS aims to use a weighted average of two $3^{\text {rd }}$ order and one $4^{\text {th }}$ order approximations for the numerical flux where each approximation involves the primitive function $H$ and its derivative at different points. To obtain the approximations for $H^{\prime}{ }_{j+\frac{1}{2}}$, three candidate stencils, 


$$
S^{0}=\left\{H_{j-\frac{3}{2}}, H_{j-\frac{1}{2}}, H_{j+\frac{1}{2}}\right\}, S^{1}=\left\{H_{j-\frac{1}{2}}, H_{j+\frac{1}{2}}, H_{j+\frac{3}{2}}\right\} \text {, and } S^{2}=\left\{H_{j+\frac{1}{2}}, H_{j+\frac{3}{2}}, H_{j+\frac{5}{2}}\right\},
$$

are used as shown in figure 3.4.

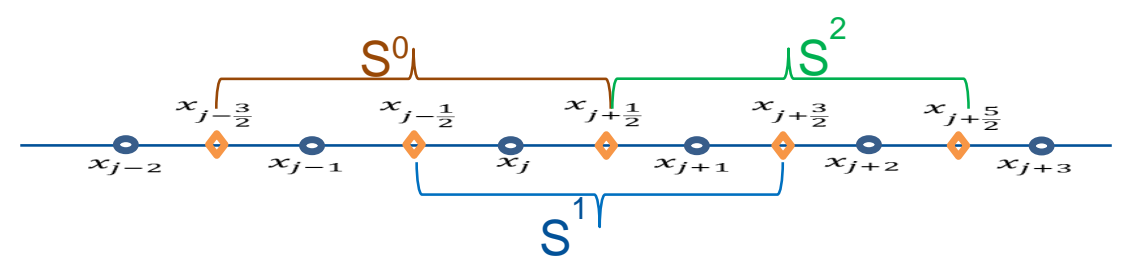

Figure 3.4. The stencils for WCS.

Similar to the WENO scheme, three candidates from the three stencils can be constructed as follows:

$$
\begin{array}{lll}
S^{0}: & 2 H^{\prime}{ }_{j-\frac{1}{2}}+H^{\prime}{ }_{j+\frac{1}{2}}=\frac{1}{h}\left(-\frac{1}{2} H_{j-\frac{3}{2}}-2 H_{j-\frac{1}{2}}+\frac{5}{2} H_{j+\frac{1}{2}}\right) & \left(3^{\text {rd }} \text { order }\right) \\
S^{1}: & \frac{1}{4} H^{\prime}{ }_{j-\frac{1}{2}}+H^{\prime}{ }_{j+\frac{1}{2}}+\frac{1}{4} H^{\prime}{ }_{j+\frac{3}{2}}=\frac{3}{4 h}\left(H_{j-\frac{3}{2}}-H_{j-\frac{1}{2}}\right) & \left(4^{\text {th }} \text { order }\right) \\
S^{2}: & H^{\prime}{ }_{j+\frac{1}{2}}+2 H^{\prime}{ }_{j+\frac{3}{2}}=\frac{1}{h}\left(_{2} H_{j+\frac{5}{2}}-2 H_{j+\frac{3}{2}}-\frac{5}{2} H_{j+\frac{1}{2}}\right) \quad\left(3^{\text {rd }} \text { order }\right)
\end{array}
$$

If a weighted average of the three stencils is considered with different constant weights, we have $\quad S=c^{0} S^{0}+c^{1} S^{1}+c^{2} S^{2}$

$$
\text { such that } \quad c^{0}=\frac{1}{18}, c^{1}=\frac{8}{9}, \text { and } c^{2}=\frac{1}{18}
$$

For consistency of the scheme, we must satisfy the equation $\sum_{i=0}^{2} c^{\mathrm{i}}=1$.

The scheme resulting from the weighted average of the three candidates is

$$
\frac{1}{3} H^{\prime}{ }_{j-\frac{1}{2}}+H^{\prime}{ }_{j+\frac{1}{2}}+\frac{1}{3} H^{\prime}{ }_{j+\frac{3}{2}} \approx \frac{1}{h}\left(\frac{23}{36} H_{j-\frac{3}{2}}-\frac{7}{9} H_{j-\frac{1}{2}}-\frac{1}{9} H_{j+\frac{3}{2}}+\frac{1}{36} H_{j+\frac{5}{2}}\right)
$$

By using the Taylor expansion, it can be verified that the scheme is the standard $6^{\text {th }}$ order compact scheme as follows: 


$$
\begin{gathered}
\frac{1}{3} H_{j-1}^{\prime}+H_{j}^{\prime}+\frac{1}{3} H_{j+1}^{\prime}-\frac{1}{h}\left(-\frac{1}{36} H_{j-2}-\frac{7}{9} H_{j-1}+\frac{7}{9} H_{j+1}+\frac{1}{36} H_{j+2}\right) \\
=-\frac{1}{1260} h^{6} F_{j}^{(7)}-\frac{1}{15120} h^{8} F_{j}^{(9)}+\cdots
\end{gathered}
$$

\subsubsection{Modified Upwinding Compact Scheme (MUCS)}

The main idea of MUCS is using the WENO scheme to improve a $7^{\text {th }}$ order upwinding compact scheme by using a new shock detector to find the shock location and using a new control function to mix the upwinding compact scheme with the WENO scheme near the shock. The reason for using the upwind technology is to introduce some numerical dissipation because the standard compact scheme has no dissipation. To obtain the $7^{\text {th }}$ order upwinding compact scheme (UCS), three $3^{\text {rd }}$ order approximations of the linear

compact combination of numerical fluxes at $\hat{F}_{j-\frac{3}{2}}, \hat{F}_{j-\frac{1}{2}}, \hat{F}_{j+\frac{1}{2}}, \hat{F}_{j+\frac{3}{2}}, \hat{F}_{j+\frac{5}{2}}$ are obtained from the three candidate stencils

$$
S^{0}=\left\{F_{j-2}, F_{j-1}, F_{j}\right\}, S^{1}=\left\{F_{j-1}, F_{j}, F_{j+1}\right\}, \text { and } S^{2}=\left\{F_{j}, F_{j+1}, F_{j+2}\right\}
$$

The $7^{\text {th }}$ order upwinding compact scheme (UCS) is

$$
\frac{1}{2} H^{\prime}{ }_{j-\frac{3}{2}}+H_{j-\frac{1}{2}}^{\prime}+\frac{1}{4} H^{\prime}{ }_{j+\frac{1}{2}}=\frac{1}{h}\left(\frac{1}{240} H_{j-\frac{7}{2}}-\frac{1}{12} H_{j-\frac{5}{2}}-\frac{11}{12} H_{j-\frac{3}{2}}+\frac{1}{3} H_{j-\frac{1}{2}}+\frac{31}{48} H_{j+\frac{1}{2}}+\frac{1}{60} H_{j+\frac{3}{2}}\right)
$$

MUCS makes the UCS able to capture the shock and keep a high order accuracy with high resolution in the smooth areas. The shock detector is a switch function that gives one for shocks and zero for others. The new control function $\Omega$ is defined as

$$
\Omega * \mathrm{UCS}+(1-\Omega) * \mathrm{WENO}
$$




\subsubsection{Modified Weighted Compact Scheme (MWCS)}

In MWCS, the WENO scheme and WCS are combined by a mixing function, which aims to linearly combine the two schemes in order to gain a numerical stability, a sharp shockcapturing, and a good resolution for small length scales. The final formulation of the numerical flux of MWCS is

$$
\widehat{F}_{j \mp \frac{1}{2}}^{(M W C S)}=\left(1-\propto_{j}\right) \hat{F}_{j \mp \frac{1}{2}}^{(W C S)}+\propto_{j} \hat{F}_{j \mp \frac{1}{2}}^{(W E N O)}
$$

where

$$
\propto_{j}=(0.5)^{*}\left(1-\frac{\left(I S^{0}-I S^{1}\right)^{2}+\left(I S^{1}-I S^{2}\right)^{2}+\left(I S^{2}-I S^{0}\right)^{2}}{2 *\left(\left(I S^{0}\right)^{2}+\left(I S^{1}\right)^{2}+\left(I S^{2}\right)^{2}\right)}\right.
$$

and $I S^{i}(\mathrm{i}=0,1,2)$ are the smoothness indicators (3.23) obtained from the WENO

scheme. Also, for consistency of the scheme, we must satisfy $0 \leq \propto_{j} \leq 1$

\subsection{Weighted Upwinding Compact Scheme (WUCS)}

\subsubsection{Basic formulation of WUCS}

For a given point $\mathrm{j}$, three candidate stencils containing these points are defined as follows:

$$
\begin{aligned}
& S^{0}=\left\{f_{j-2}, f_{j-1}, f_{j}\right\} \\
& S^{1}=\left\{f_{j-1}, f_{j}, f_{j+1}\right\} \\
& S^{2}=\left\{f_{j}, f_{j+1}, f_{j+2}\right\}
\end{aligned}
$$

Below are the schemes for the three candidate stencils, which are calculated by repeating the steps applied to (3.11) at each stencil.

$$
S^{0}: \hat{f}_{j-\frac{3}{2}}+2 \hat{f}_{j-\frac{1}{2}}+\frac{1}{3} \hat{f}_{j+\frac{1}{2}}=\frac{1}{9} f_{j-2}+\frac{19}{9} f_{j-1}+\frac{10}{9} f_{j}
$$




$$
\begin{gathered}
S^{1}:-\hat{f}_{j-\frac{1}{2}}-2 \hat{f}_{j+\frac{1}{2}}-\frac{1}{3} \hat{f}_{j+\frac{3}{2}}=-\frac{1}{9} f_{j-1}-\frac{19}{9} f_{j}-\frac{10}{9} f_{j+1} \\
S^{2}:-\hat{f}_{j-\frac{1}{2}}-6 \hat{f}_{j+\frac{1}{2}}-3 \hat{f}_{j+\frac{3}{2}}=-\frac{10}{3} f_{j}-\frac{19}{3} f_{j+1}-\frac{1}{3} f_{j+2}
\end{gathered}
$$

All three schemes above are a $5^{\text {th }}$ order. According to the idea of the WENO scheme, a new $7^{\text {th }}$ order scheme is obtained by a linear combination of all stencils with a specific weight assigned to each one of them. Consider $c^{0}=\frac{3}{13}, c^{1}=\frac{33}{52}$, and $c^{2}=\frac{7}{52}$ are the weights assigned to the stencils $S^{0}, S^{1}$, and $S^{2}$ respectively. In addition, for consistency of the scheme, the sum of these weights should be equal to one. Hence, from the combination

$$
S=c^{0} S^{0}+c^{1} S^{1}+c^{2} S^{2},
$$

the final scheme for the positive primitive function $\mathrm{H}^{+}$can be written as follows:

$$
\frac{3}{13} \hat{f}_{j-\frac{3}{2}}-\frac{4}{13} \hat{f}_{j-\frac{1}{2}}-2 \hat{f}_{j+\frac{1}{2}}-\frac{8}{13} \hat{f}_{j+\frac{3}{2}}=\frac{1}{39} f_{j-2}+\frac{5}{12} f_{j-1}-\frac{239}{156} f_{j}-\frac{81}{52} f_{j+1}-\frac{7}{156} f_{j+2}
$$

For the negative primitive function $H^{-}$, the formula is similar. It can be verified that the scheme above has a $7^{\text {th }}$ order by using the Taylor expansion for each term. In addition, the numerical results in chapter 4 show the scheme has $7^{\text {th }}$ order with high resolution.

\subsubsection{Boundary points schemes}

Special $7^{\text {th }}$ order schemes are constructed for boundary the points as follows:

Point 0,

$$
\hat{f}_{j-\frac{1}{2}}=\frac{363}{140} f_{j}-\frac{617}{140} f_{j+1}+\frac{853}{140} f_{j+2}-\frac{2341}{420} f_{j+3}+\frac{667}{210} f_{j+4}-\frac{43}{42} f_{j+5}+\frac{1}{7} f_{j+6}
$$


Point 1,

$$
\hat{f}_{j-\frac{1}{2}}=\frac{1}{7} f_{j-1}+\frac{223}{140} f_{j}-\frac{197}{140} f_{j+1}+\frac{153}{140} f_{j+2}-\frac{241}{420} f_{j+3}+\frac{37}{210} f_{j+4}-\frac{1}{42} f_{j+5}
$$

Point 2,

$$
\hat{f}_{j-\frac{1}{2}}=-\frac{1}{42} f_{j-2}+\frac{13}{42} f_{j-1}+\frac{153}{140} f_{j}-\frac{241}{420} f_{j+1}+\frac{109}{140} f_{j+2}-\frac{31}{420} f_{j+3}+\frac{1}{105} f_{j+4}
$$

Point N-1,

$$
\hat{f}_{j+\frac{1}{2}}=-\frac{1}{42} f_{j-5}+\frac{37}{210} f_{j-4}-\frac{241}{420} f_{j-3}+\frac{153}{140} f_{j-2}-\frac{197}{140} f_{j-1}+\frac{223}{140} f_{j}+\frac{1}{7} f_{j+1}
$$

Point N,

$$
\hat{f}_{j+\frac{1}{2}}=\frac{1}{7} f_{j-6}-\frac{43}{42} f_{j-5}+\frac{667}{210} f_{j-4}-\frac{2341}{420} f_{j-3}+\frac{853}{140} f_{j-2}-\frac{617}{140} f_{j-1}+\frac{363}{140} f_{j}
$$

\subsection{Dispersion and Dissipation Analysis}

The dispersion and dissipation errors are efficiently quantified using the Fourier analysis by R. Vichnevetsky (1982) and J. Anderson (1995). Hence, the resolution and diffusion properties of the WUCS scheme are provided by the Fourier analysis and compared with the WENO scheme and WCS. Since the Fourier analysis requires periodicity, the dependent variables are considered to be periodic over the domain [0 , L] of independent variables. $f_{1}=f_{N+1}$ and $h=\frac{L}{N}$. The dependent variables can be written in terms of the Fourier coefficients as follows:

$$
f(x)=\sum_{k=-\frac{N}{2}}^{\frac{N}{2}} \hat{f}_{k} e^{\frac{2 \pi i k x}{L}}
$$

Where $i=\sqrt{-1}$ and $x \in[0, L]$. The Fourier coefficients satisfy that $\hat{f}_{0}=\overline{\hat{f}}_{0}$ and 
$\hat{f}_{k}=\hat{f}_{-k}$ for $1 \leq \mathrm{k} \leq \frac{N}{2}$ because the dependent variables are real values. For programing convenience, a scaled wave number $w=\frac{2 \pi k h}{L}=\frac{2 \pi k}{N}$ and a scaled coordinate $s=\frac{x}{h}$ are introduced. Hence, (3.39) becomes

$$
f(x)=f(s h)=\sum_{k=-\frac{N}{2}}^{\frac{N}{2}} \hat{f}_{k} e^{i w s}
$$

The domain of the scaled wave number $\mathrm{w}$ is $[0, \pi]$. The first derivative of (3.40) can be computed with respect of $s$ as follows:

$$
h f^{\prime}(s h)=\sum_{k=-\frac{N}{2}}^{\frac{N}{2}} i w \hat{f}_{k} e^{i w s}
$$

When comparing the Fourier coefficients of the derivative obtained from the differencing scheme $\left(\hat{f}_{k}^{\prime}\right)_{f d}$ with the exact Fourier coefficients, $\hat{f}_{k}^{\prime}$, the differencing errors can be obtained. In general, a finite difference scheme corresponds to a function of $\mathrm{w}, w^{\prime}(w)$, which is called the effective wave number. The straight line $w^{\prime}(w)=w$ represents the exact differentiation. The real part of the effective wave number $\left(w^{\prime}\right)$ quantifies the dissipation error while the dispersion error, which represents the resolution of the scheme, can be quantified by the imaginary part of $w^{\prime}$. The dissipation errors constitute the amplification introduced by the scheme while the dispersion errors represent the waves for different wave numbers traveling at different velocities. The dispersion and dissipation of the WENO scheme, WCS, and WUCS are discussed in the following subsections at each stencil (for non-smooth areas) and at the combinations of all stencils (for smooth areas). 


\subsubsection{Dispersion and dissipation analysis for the left stencils $\left(S^{0}\right)$}

One problem with the WCS scheme (Jiang et al., 2001) is it has a negative dissipation for the left candidate, and this problem is treated when constructing the WUCS scheme. The scheme for the left stencil of WUCS is given in (3.35) as follows:

$$
\hat{f}_{j-\frac{3}{2}}+2 \hat{f}_{j-\frac{1}{2}}+\frac{1}{3} \hat{f}_{j+\frac{1}{2}}=\frac{1}{9} f_{j-2}+\frac{19}{9} f_{j-1}+\frac{10}{9} f_{j}
$$

First, the above scheme can be rewritten in terms of $\mathrm{H}$ when substituting (3.7) and (3.8) in (3.35) as follows:

$$
h\left(H_{j-\frac{3}{2}}^{\prime}+2 H_{j-\frac{1}{2}}^{\prime}+\frac{1}{3} H_{j+\frac{1}{2}}^{\prime}\right)=\frac{1}{9}\left(H_{j-\frac{3}{2}}-H_{j-\frac{5}{2}}\right)+\frac{19}{9}\left(H_{j-\frac{1}{2}}-H_{j-\frac{3}{2}}\right)+\frac{10}{9}\left(H_{j+\frac{1}{2}}-H_{j-\frac{1}{2}}\right)
$$

For simplification, letting $\mathrm{j}=0$ and shifting the scheme by $-\frac{1}{2}$ gives

$$
h\left(H_{-2}^{\prime}+2 H_{-1}^{\prime}+\frac{1}{3} H_{0}^{\prime}\right)=\frac{1}{9}\left(H_{-2}-H_{-3}\right)+\frac{19}{9}\left(H_{-1}-H_{-2}\right)+\frac{10}{9}\left(H_{0}-H_{1}\right)
$$

From (3.40) and (3.41), we have

$$
\begin{gathered}
H_{j}=\sum_{k=-\frac{N}{2}}^{\frac{N}{2}} \widehat{H}_{k} e^{i w j} \\
H^{\prime}{ }_{j}=\sum_{k=-\frac{N}{2}}^{\frac{N}{2}} i w \widehat{H}_{k} e^{i w j}
\end{gathered}
$$

Substituting (3.43) and (3.44) in (3.42) results in
$\mathrm{h} \sum_{k=-\frac{N}{2}}^{\frac{N}{2}} \widehat{H}_{k}\left(i w e^{-2 i w}+2 i w e^{-i w}+\frac{i w}{3}\right)=\sum_{k=-\frac{N}{2}}^{\frac{N}{2}} \widehat{H}_{k}\left(\frac{1}{9}\left(e^{-2 i w}-e^{-3 i w}\right)+\frac{19}{9}\left(e^{-i w}-e^{-2 i w}\right)+\frac{10}{9}\left(1-e^{-i w}\right)\right)$
$\mathrm{h} \sum_{k=-\frac{N}{2}}^{\frac{N}{2}} \widehat{H}_{k}\left[\left(i w e^{-2 i w}+2 i w e^{-i w}+\frac{i w}{3}\right)-\left(\frac{1}{9}\left(e^{-2 i w}-e^{-3 i w}\right)+\frac{19}{9}\left(e^{-i w}-e^{-2 i w}\right)+\frac{10}{9}\left(1-e^{-i w}\right)\right)\right]=0$ 


$$
\begin{gathered}
{\left[\left(i w e^{-2 i w}+2 i w e^{-i w}+\frac{i w}{3}\right)-\left(\frac{1}{9}\left(e^{-2 i w}-e^{-3 i w}\right)+\frac{19}{9}\left(e^{-i w}-e^{-2 i w}\right)+\frac{10}{9}\left(1-e^{-i w}\right)\right)\right] \mathrm{h} \sum_{k=-\frac{N}{2}}^{\frac{N}{2}} \widehat{H}_{k}=0} \\
{\left[\left(i w e^{-2 i w}+2 i w e^{-i w}+\frac{i w}{3}\right)-\left(\frac{1}{9}\left(e^{-2 i w}-e^{-3 i w}\right)+\frac{19}{9}\left(e^{-i w}-e^{-2 i w}\right)+\frac{10}{9}\left(1-e^{-i w}\right)\right)\right]=0} \\
\left(i w e^{-2 i w}+2 i w e^{-i w}+\frac{i w}{3}\right)=\frac{1}{9}\left(e^{-2 i w}-e^{-3 i w}\right)+\frac{19}{9}\left(e^{-i w}-e^{-2 i w}\right)+\frac{10}{9}\left(1-e^{-i w}\right)
\end{gathered}
$$

Using Euler's formula, $e^{i w}=\operatorname{Cos}[w]+i \operatorname{Sin}[w]$, results in

$$
\begin{gathered}
2 h w \operatorname{Sin}[w]+h w \operatorname{Sin}[2 w]+i\left(\frac{h w}{3}+2 h w \operatorname{Cos}[w]+h w \operatorname{Cos}[2 w]\right) \\
=\frac{10}{9}+\operatorname{Cos}[w]-2 \operatorname{Cos}[2 w]-\frac{1}{9} \operatorname{Cos}[3 w]+i\left(-\operatorname{Sin}[w]+2 \operatorname{Sin}[2 w]+\frac{1}{9} \operatorname{Sin}[3 w]\right) \\
h w \sin (2 w)+2 h w \sin (w)+i\left(h w \cos (2 w)+2 h w \operatorname{Cos}[w]+\frac{h w}{3}\right) \\
=-\frac{1}{9} \cos (3 w)-2 \operatorname{Cos}(2 w)+\frac{10}{9}+i\left(\frac{1}{9} \sin (3 w)+2 \sin (2 w)-\sin (w)\right) \\
w=\frac{-10 i-9 i \operatorname{Cos}[w]+18 i \operatorname{Cos}[2 w]+i \operatorname{Cos}[3 w]-9 \operatorname{Sin}[w]+18 \operatorname{Sin}[2 w]+\operatorname{Sin}[3 w]}{3 h(1+6 \operatorname{Cos}[w]+3 \operatorname{Cos}[2 w]-6 i \operatorname{Sin}[w]-3 i \operatorname{Sin}[2 w])} \\
w=\frac{-9 \mathrm{i}+8 \mathrm{i} \operatorname{Cos}[w]+\mathrm{i} \operatorname{Cos}[2 w]+28 \operatorname{Sin}[w]+\operatorname{Sin}[2 w]}{6 h(3+2 \operatorname{Cos}[w]-\mathrm{i} \operatorname{Sin}[w])}
\end{gathered}
$$

Letting $h=1$ gives

$$
w=\frac{-9 i+8 i \operatorname{Cos}[w]+i \operatorname{Cos}[2 w]+28 \operatorname{Sin}[w]+\operatorname{Sin}[2 w]}{6(3+2 \operatorname{Cos}[w]-i \operatorname{Sin}[w])}
$$

$w$ in (3.45) represents the wave number for the left stencil of WUCS, and it is denoted by $w_{(W U C S)}$.

Hence, $\quad w_{(W U C S)}=\frac{-9 i+8 i \operatorname{Cos}[w]+i \operatorname{Cos}[2 w]+28 \operatorname{Sin}[w]+\operatorname{Sin}[2 w]}{6(3+2 \operatorname{Cos}[w]-i \operatorname{Sin}[w])}$ 
Similarly, the wave numbers for the left stencils of WCS and the WENO scheme can be computed as follows:

$$
w_{(W C S)}=\frac{2 i-2 i \operatorname{Cos}[w]+3 \operatorname{Sin}[w]}{2+\operatorname{Cos}[w]+i \operatorname{Sin}[w]}
$$

and

$$
W_{(W E N O)}=\frac{i(-11+18 \operatorname{Cos}[w]-9 \operatorname{Cos}[2 w]+2 \operatorname{Cos}[3 w])+18 \operatorname{Sin}[w]-9 \operatorname{Sin}[2 w]+2 \operatorname{Sin}[3 w]}{6}
$$

Now, the dispersion and dissipation errors can be found as follows:

Effective Wave Number $\left(w^{\prime}\right)=$ Dissipation $+i^{*}$ Dispersion

Effective Wave Number $\left(w^{\prime}\right)=i w$

let $\quad w=a+i b$

so $\quad w^{\prime}=i(a+i b)=i a-b=-b+i a$

The Dispersion $=a=\operatorname{Re}(\mathrm{w})$

The Dissipation $=-\mathrm{b}=-\operatorname{Im}(\mathrm{w})$

The dispersion errors of WUCS, WCS, and the WENO scheme are

$$
\begin{aligned}
\operatorname{Re}\left(w_{(W U C S)}\right) & =\operatorname{Re}\left(\frac{-9 \mathrm{i}+8 \mathrm{i} \operatorname{Cos}[w]+\mathrm{i} \operatorname{Cos}[2 w]+28 \operatorname{Sin}[w]+\operatorname{Sin}[2 w]}{6(3+2 \operatorname{Cos}[w]-\mathrm{i} \operatorname{Sin}[w])}\right) \\
\operatorname{Re}\left(w_{(\mathrm{WCS})}\right)= & \operatorname{Re}\left(\frac{2 \mathrm{i}-2 \mathrm{i} \operatorname{Cos}[w]+3 \operatorname{Sin}[w]}{2+\operatorname{Cos}[w]+i \operatorname{Sin}[w]}\right) \\
& =\frac{(8+\operatorname{Cos}[w]) \operatorname{Sin}[w]}{5+4 \operatorname{Cos}[w]}, \text { and } \\
\operatorname{Re}\left(W_{(W E N O)}\right)= & \operatorname{Re}\left(\frac{\mathrm{i}(-11+18 \operatorname{Cos}[w]-9 \operatorname{Cos}[2 w]+2 \operatorname{Cos}[3 w])+18 \operatorname{Sin}[w]-9 \operatorname{Sin}[2 w]+2 \operatorname{Sin}[3 w]}{6}\right) \\
= & \frac{18 \operatorname{Sin}[w]-9 \operatorname{Sin}[2 w]+2 \operatorname{Sin}[3 w]}{6}
\end{aligned}
$$




$$
=3 \operatorname{Sin}[w]-\frac{3}{2} \operatorname{Sin}[2 w]+\frac{1}{3} \operatorname{Sin}[3 w]
$$

As shown in Figure 3.5, the WENO scheme has the biggest dispersion errors among the three schemes, so it has the lowest resolution. Both WUCS and WCS have high resolution with close dispersion errors.

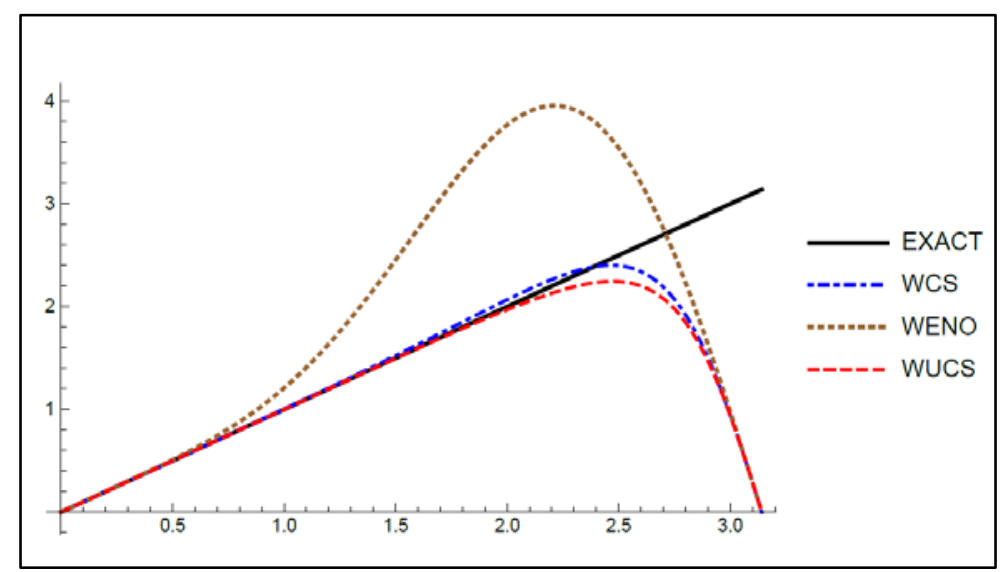

Figure 3.5. The dispersion in the left stencils of WUCS, WCS, and the WENO scheme.

The dissipation errors are

$$
\begin{aligned}
-\operatorname{Im}\left(w_{(W U C S)}\right) & =-\operatorname{Im}\left(\frac{-9 \mathrm{i}+8 \mathrm{i} \operatorname{Cos}[w]+\mathrm{i} \operatorname{Cos}[2 w]+28 \operatorname{Sin}[w]+\operatorname{Sin}[2 w]}{6(3+2 \operatorname{Cos}[w]-\mathrm{i} \operatorname{Sin}[w])}\right) \\
-\operatorname{Im}\left(w_{(\mathrm{WCS})}\right) & =-\operatorname{Im}\left(\frac{2 i-2 i \operatorname{Cos}[w]+3 \operatorname{Sin}[w]}{2+\operatorname{Cos}[w]+i \operatorname{Sin}[w]}\right) \\
& =-\frac{4 \operatorname{Sin}\left[\frac{w}{2}\right]^{4}}{5+4 \operatorname{Cos}[w]}, \text { and } \\
-\operatorname{Im}\left(W_{(W E N O)}\right) & =-\operatorname{Im}\left(\frac{i(-11+18 \operatorname{Cos}[w]-9 \operatorname{Cos}[2 w]+2 \operatorname{Cos}[3 w])+18 \operatorname{Sin}[w]-9 \operatorname{Sin}[2 w]+2 \operatorname{Sin}[3 w]}{6}\right) \\
= & -\left(\frac{(-11+18 \operatorname{Cos}[w]-9 \operatorname{Cos}[2 w]+2 \operatorname{Cos}[3 w])}{6}\right) \\
= & \frac{11}{6}-3 \operatorname{Cos}[w]+\frac{3}{2} \operatorname{Cos}[2 w]-\frac{1}{3} \operatorname{Cos}[3 w]
\end{aligned}
$$


Figure 3.6 illustrates that WUCS has a positive dissipation while WCS has a negative dissipation, and the WENO scheme fluctuates over the wave number range. Also, the dissipation errors in WUCS are the lowest compared to the WENO scheme and WCS.

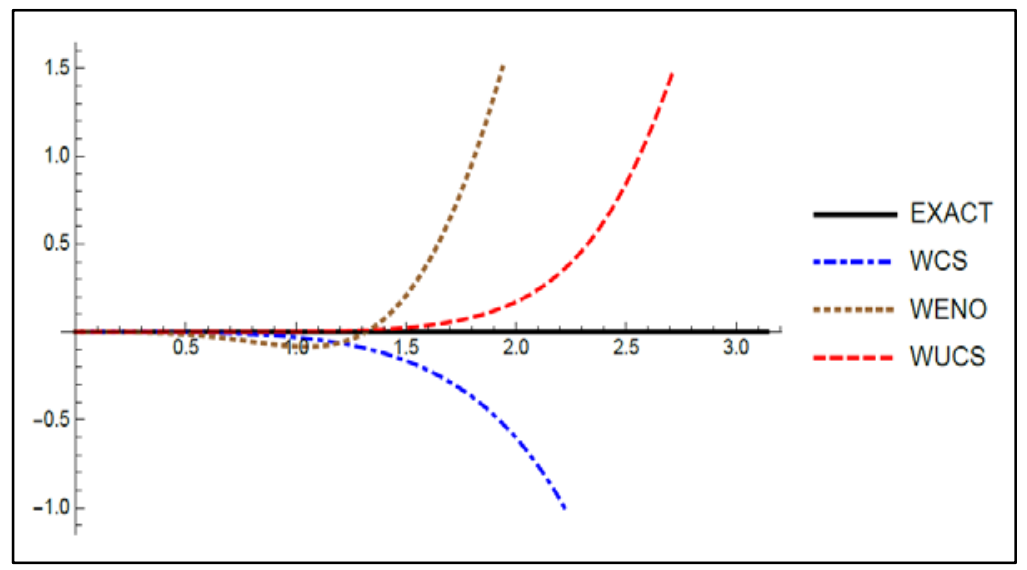

Figure 3.6. Dissipation in the left stencils of WUCS, WCS, and the WENO scheme.

\subsubsection{Dispersion and dissipation analysis for the center stencils $\left(S^{1}\right)$}

The scheme for the center stencil of WUCS is given in (3.36) as follows:

$$
-\hat{f}_{j-\frac{1}{2}}-2 \hat{f}_{j+\frac{1}{2}}-\frac{1}{3} \hat{f}_{j+\frac{3}{2}}=-\frac{1}{9} f_{j-1}-\frac{19}{9} f_{j}-\frac{10}{9} f_{j+1}
$$

Applying the same procedure and analysis from section 3.3.1 results in

$$
h\left(-2 i w-i e^{-i w} w-\frac{1}{3} i e^{i w} w\right)=-\frac{19}{9}\left(1-e^{-i w}\right)-\frac{10}{9}\left(-1+e^{i w}\right)+\frac{1}{9}\left(-e^{-i w}+e^{-2 i w}\right)
$$

By letting $h=1$ and using Euler's formula, we arrive at

$$
i\left(-2 w-\frac{4}{3} w \operatorname{Cos}[w]\right)-\frac{2}{3} h w \operatorname{Sin}[w]=-1+\frac{8}{9} \operatorname{Cos}[w]+\frac{1}{9} \operatorname{Cos}[2 w]+i\left(-\frac{28}{9} \operatorname{Sin}[w]-\frac{1}{9} \operatorname{Sin}[2 w]\right) w
$$




$$
w=\frac{-9 i+8 i \operatorname{Cos}[w]+i \operatorname{Cos}[2 w]+28 \operatorname{Sin}[w]+\operatorname{Sin}[2 w]}{6(3+2 \operatorname{Cos}[w]-i \operatorname{Sin}[w])}
$$

Hence,

$$
w_{(W U C S)}=\frac{-9 \mathrm{i}+8 \mathrm{i} \operatorname{Cos}[w]+\mathrm{i} \operatorname{Cos}[2 w]+28 \operatorname{Sin}[w]+\operatorname{Sin}[2 w]}{6(3+2 \operatorname{Cos}[w]-\mathrm{i} \operatorname{Sin}[w])}
$$

Similarly, the wave numbers for the center stencils of WCS and the WENO scheme are

$$
\begin{gathered}
w_{(\mathrm{WCS})}=\frac{3 \operatorname{Sin}[w]}{2+\operatorname{Cos}[w]} \text { and } \\
W_{(W E N O)}=\frac{\mathrm{i}(-3+4 \operatorname{Cos}[w]-\operatorname{Cos}[2 w])+8 \operatorname{Sin}[w]-\operatorname{Sin}[2 w]}{6}
\end{gathered}
$$

The dispersion errors of WUCS, WCS, and WENO are

$$
\begin{aligned}
\operatorname{Re}\left(w_{(W U C S)}\right) & =\operatorname{Re}\left(\frac{-9 \mathrm{i}+8 \mathrm{i} \operatorname{Cos}[w]+\mathrm{i} \operatorname{Cos}[2 w]+28 \operatorname{Sin}[w]+\operatorname{Sin}[2 w]}{6(3+2 \operatorname{Cos}[w]-\mathrm{i} \operatorname{Sin}[w])}\right) \\
& =\frac{189 \operatorname{Sin}[w]+54 \operatorname{Sin}[2 w]+\operatorname{Sin}[3 w]}{6(23+24 \operatorname{Cos}[w]+3 \operatorname{Cos}[2 w])} \\
\operatorname{Re}\left(w_{(\mathrm{WCS})}\right) & =\operatorname{Re}\left(\frac{3 \operatorname{Sin}[w]}{2+\operatorname{Cos}[w]}\right)=\frac{3 \operatorname{Sin}[w]}{2+\operatorname{Cos}[w]}, \text { and } \\
\operatorname{Re}\left(W_{(W E N O)}\right) & =\operatorname{Re}\left(\frac{i(-3+4 \operatorname{Cos}[w]-\operatorname{Cos}[2 w])+8 \operatorname{Sin}[w]-\operatorname{Sin}[2 w]}{6}\right) \\
& =\frac{8 \operatorname{Sin}[w]-\operatorname{Sin}[2 w]}{6} \\
= & \frac{4}{3} \operatorname{Sin}[w]-\frac{1}{6} \operatorname{Sin}[2 w]
\end{aligned}
$$

As illustrated in Figure 3.7, the resolution of WCS is better than the resolution of the WENO scheme, and WUCS is the best among all of them because it is of the lowest dispersion errors. 


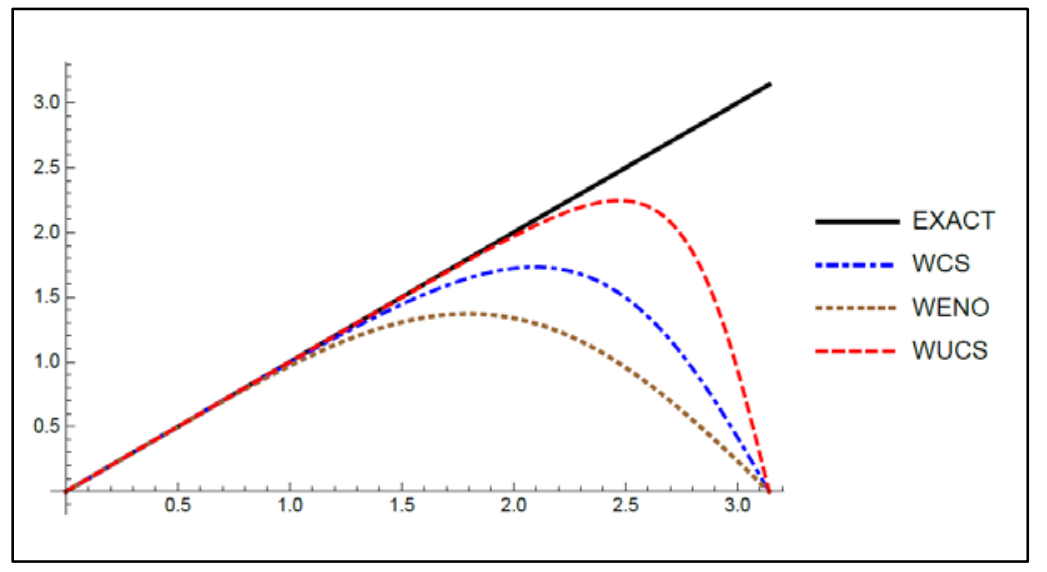

Figure 3.7. The dispersion in the center stencils of WUCS, WCS, and the WENO scheme.

The dissipation errors are

$$
\begin{aligned}
-\operatorname{Im}\left(w_{(\text {WUCS })}\right) & =-\operatorname{Im}\left(\frac{189 \operatorname{Sin}[w]+54 \operatorname{Sin}[2 w]+\operatorname{Sin}[3 w]}{6(23+24 \operatorname{Cos}[w]+3 \operatorname{Cos}[2 w])}\right) \\
& =\frac{16 \operatorname{Sin}\left[\frac{w}{2}\right]^{6}}{69+72 \operatorname{Cos}[w]+9 \operatorname{Cos}[2 w]}, \\
-\operatorname{Im}\left(w_{(\text {WCS })}\right) & =-\operatorname{Im}\left(\frac{3 \operatorname{Sin}[w]}{2+\operatorname{Cos}[w]}\right)=0, \text { and } \\
-\operatorname{Im}\left(W_{(W E N O)}\right) & =-\operatorname{Im}\left(\frac{i(-3+4 \operatorname{Cos}[w]-\operatorname{Cos}[2 w])+8 \operatorname{Sin}[w]-\operatorname{Sin}[2 w]}{6}\right) \\
& =-\left(\frac{(-3+4 \operatorname{Cos}[w]-\operatorname{Cos}[2 w])}{6}\right) \\
& =\frac{1}{2}-\frac{2}{3} \operatorname{Cos}[w]+\frac{1}{6} \operatorname{Cos}[2 w]
\end{aligned}
$$

Figure 3.8 represents that both the WENO scheme and WUCS have a positive dissipation while WCS has no dissipation. Also, the dissipation errors in WUCS are much fewer than the dissipation errors in the WENO scheme. 


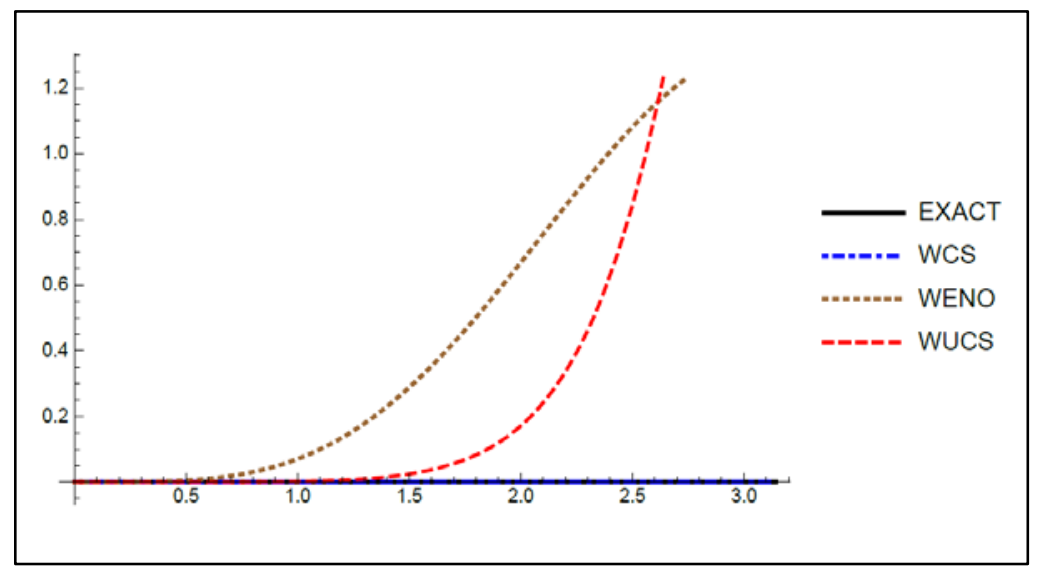

Figure 3.8. The dissipation in the center stencils of

WUCS, WCS, and the WENO scheme.

\subsubsection{Dispersion and dissipation analysis for the right stencils $\left(S^{2}\right)$}

The scheme for the right stencil of WUCS is given in (3.37) as follows:

$$
-\hat{f}_{j-\frac{1}{2}}-6 \hat{f}_{j+\frac{1}{2}}-3 \hat{f}_{j+\frac{3}{2}}=-\frac{10}{3} f_{j}-\frac{19}{3} f_{j+1}-\frac{1}{3} f_{j+2}
$$

Applying the process in section 3.3.1 results in

$$
\begin{gathered}
h\left(-6 i \mathrm{w}-i e^{-i w} \mathrm{w}-3 i e^{i w} \mathrm{w}\right)=-\frac{10}{3}\left(1-e^{-i w}\right)-\frac{19}{3}\left(-1+e^{i w}\right)+\frac{1}{3}\left(e^{i w}-e^{2 i w}\right) \\
i(-6 h \mathrm{w}-4 h \mathrm{w} \operatorname{Cos}[w])+2 h \mathrm{w} \operatorname{Sin}[w]==3-\frac{8}{3} \operatorname{Cos}[w]-\frac{1}{3} \operatorname{Cos}[2 w]+i\left(-\frac{28}{3} \operatorname{Sin}[w]-\frac{1}{3} \operatorname{Sin}[2 w]\right)
\end{gathered}
$$

Letting $h=1$ and using Euler's formula provides

$$
\begin{aligned}
& w=\frac{9 i-8 i \operatorname{Cos}[w]-i \operatorname{Cos}[2 w]+28 \operatorname{Sin}[w]+\operatorname{Sin}[2 w]}{6(3+2 \operatorname{Cos}[w]+i \operatorname{Sin}[w])} \\
& w=\frac{9 i-8 i \operatorname{Cos}[w]-i \operatorname{Cos}[2 w]+28 \operatorname{Sin}[w]+\operatorname{Sin}[2 w]}{18+12 \operatorname{Cos}[w]+6 i \operatorname{Sin}[w]}
\end{aligned}
$$


Hence,

$$
w_{(W U C S)}=\frac{9 i-8 i \operatorname{Cos}[w]-i \operatorname{Cos}[2 w]+28 \operatorname{Sin}[w]+\operatorname{Sin}[2 w]}{18+12 \operatorname{Cos}[w]+6 i \operatorname{Sin}[w]}
$$

Similarly, the wave numbers for the right stencils of the WENO scheme and WCS are

$$
\begin{gathered}
w_{(W C S)}=\frac{5 i-4 i \operatorname{Cos}[w]-i \operatorname{Cos}[2 w]+4 \operatorname{Sin}[w]+\operatorname{Sin}[2 w]}{2(1+2 \operatorname{Cos}[w]+2 i \operatorname{Sin}[w])} \text { and } \\
W_{(W E N O)}=\frac{i(3-4 \operatorname{Cos}[w]+\operatorname{Cos}[2 w])+8 \operatorname{Sin}[w]-\operatorname{Sin}[2 w]}{6}
\end{gathered}
$$

The dispersion errors of WUCS, WCS, and the WENO scheme are

$$
\begin{gathered}
\operatorname{Re}\left(w_{(W U C S)}\right)=\operatorname{Re}\left(\frac{9 i-8 i \operatorname{Cos}[w]-i \operatorname{Cos}[2 w]+28 \operatorname{Sin}[w]+\operatorname{Sin}[2 w]}{18+12 \operatorname{Cos}[w]+6 i \operatorname{Sin}[w]}\right) \\
\operatorname{Re}\left(w_{(\mathrm{WCS})}\right)=\operatorname{Re}\left(\frac{5 i-4 i \operatorname{Cos}[w]-i \operatorname{Cos}[2 w]+4 \operatorname{Sin}[w]+\operatorname{Sin}[2 w]}{2(1+2 \operatorname{Cos}[w]+2 i \operatorname{Sin}[w])}\right) \\
=\frac{(8+\operatorname{Cos}[w]) \operatorname{Sin}[w]}{5+4 \operatorname{Cos}[w]}, \text { and } \\
\operatorname{Re}\left(W_{(W E N O)}\right)=\operatorname{Re}\left(\frac{i(3-4 \operatorname{Cos}[w]+\operatorname{Cos}[2 w])+8 \operatorname{Sin}[w]-\operatorname{Sin}[2 w]}{6}\right) \\
=\frac{8 \operatorname{Sin}[w]-\operatorname{Sin}[2 w]}{6} \\
=\frac{4}{3} \operatorname{Sin}[w]-\frac{1}{6} \operatorname{Sin}[2 w]
\end{gathered}
$$

It can be clearly seen from Figure 3.9 that both WUCS and WCS have high resolution. Also, the WENO scheme has the lowest resolution among the three schemes because it has the biggest dispersion errors. 


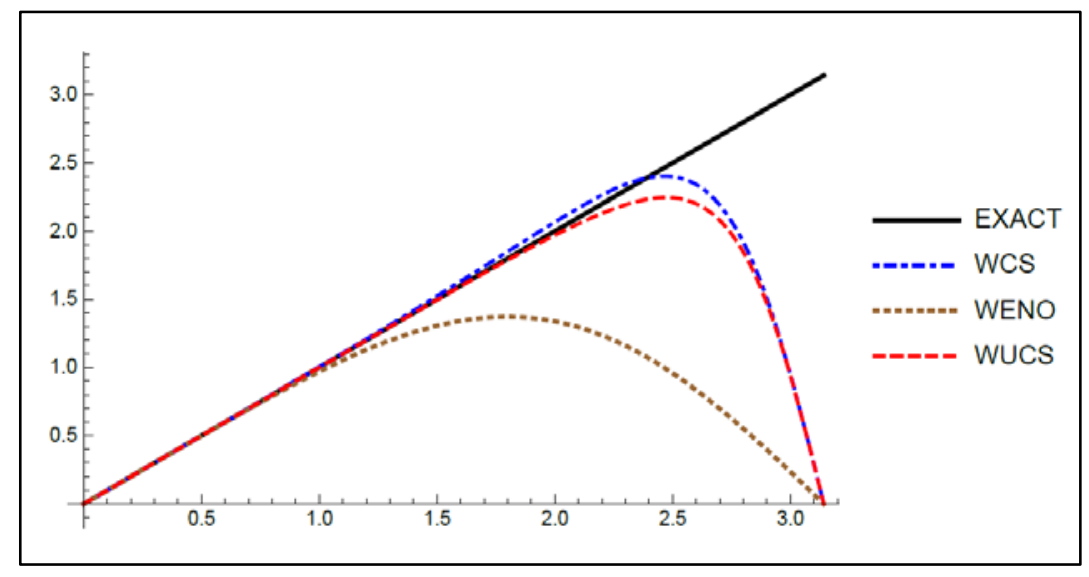

Figure 3.9. The dispersion in the right stencils of WUCS, WCS, and the WENO scheme.

The dissipation errors are

$$
\begin{aligned}
-\operatorname{Im}\left(w_{(W U C S)}\right) & =-\operatorname{Im}\left(\frac{9 i-8 i \operatorname{Cos}[w]-i \operatorname{Cos}[2 w]+28 \operatorname{Sin}[w]+\operatorname{Sin}[2 w]}{18+12 \operatorname{Cos}[w]+6 i \operatorname{Sin}[w]}\right), \\
-\operatorname{Im}\left(w_{(\mathrm{WCS})}\right) & =-\operatorname{Im}\left(\frac{5 i-4 i \operatorname{Cos}[w]-i \operatorname{Cos}[2 w]+4 \operatorname{Sin}[w]+\operatorname{Sin}[2 w]}{2(1+2 \operatorname{Cos}[w]+2 i \operatorname{Sin}[w])}\right) \\
= & \frac{4 \operatorname{Sin}\left[\frac{w}{2}\right]^{4}}{5+4 \operatorname{Cos}[w]}, \text { and } \\
-\operatorname{Im}\left(W_{(W E N O)}\right) & =-\operatorname{Im}\left(\frac{i(3-4 \operatorname{Cos}[w]+\operatorname{Cos}[2 w])+8 \operatorname{Sin}[w]-\operatorname{Sin}[2 w]}{6}\right) \\
= & -\left(\frac{(3-4 \operatorname{Cos}[w]+\operatorname{Cos}[2 w])}{6}\right) \\
= & -\frac{1}{2}+\frac{2}{3} \operatorname{Cos}[w]-\frac{1}{6} \operatorname{Cos}[2 w]
\end{aligned}
$$

Figure 3.10 illustrates that both the WENO scheme and WUCS have a negative dissipation while WCS has a positive dissipation. Also, the dissipation errors in WUCS are the lowest compared to the WENO scheme and WCS. 


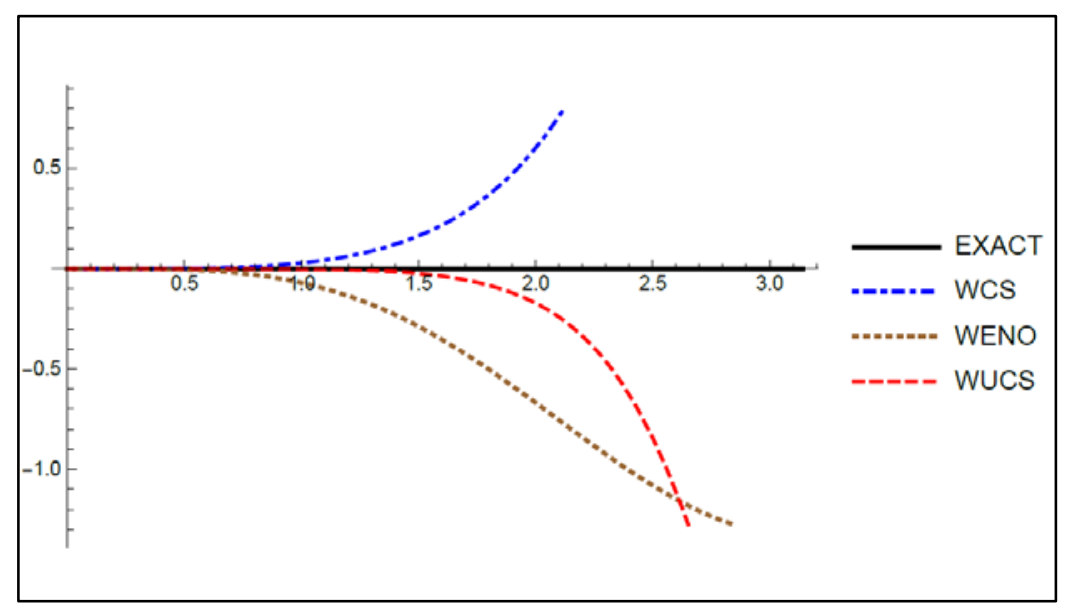

Figure 3.10. The dissipation in the right stencils of WUCS, WCS, and the WENO scheme.

\subsubsection{Dispersion and dissipation analysis for the overall schemes}

For smooth regions, the combination of all schemes from all stencils is used to get a high order scheme. The $7^{\text {th }}$ order WUCS scheme is given in (3.38) as follows:

$$
\begin{aligned}
\frac{3}{13} \hat{f}_{j-\frac{3}{2}}-\frac{4}{13} \hat{f}_{j-\frac{1}{2}}-2 \hat{f}_{j+\frac{1}{2}}-\frac{8}{13} \hat{f}_{j+\frac{3}{2}} & \\
& =\frac{1}{39} f_{j-2}+\frac{5}{12} f_{j-1}-\frac{239}{156} f_{j}-\frac{81}{52} f_{j+1}-\frac{7}{156} f_{j+2}
\end{aligned}
$$

Now, repeating the process from section 3.3.1 gives

$$
w=\frac{2 i+34 i \operatorname{Cos}[w]-34 i \operatorname{Cos}[2 w]-2 i \operatorname{Cos}[3 w]+270 \operatorname{Sin}[w]-27 \operatorname{Sin}[2 w]-2 \operatorname{Sin}[3 w]}{6(26+12 \operatorname{Cos}[w]-3 \operatorname{Cos}[2 w]+4 i \operatorname{Sin}[w]+3 i \operatorname{Sin}[2 w])}
$$

Hence,

$$
w_{(W U C S)}=\frac{2 i+34 i \operatorname{Cos}[w]-34 i \operatorname{Cos}[2 w]-2 i \operatorname{Cos}[3 w]+270 \operatorname{Sin}[w]-27 \operatorname{Sin}[2 w]-2 \operatorname{Sin}[3 w]}{6(26+12 \operatorname{Cos}[w]-3 \operatorname{Cos}[2 w]+4 i \operatorname{Sin}[w]+3 i \operatorname{Sin}[2 w])}
$$

Similarly, the wave numbers for the WENO scheme and WCS are 


$$
\begin{gathered}
w_{(\mathrm{WCS})}=\frac{(14+\operatorname{Cos}[w]) \operatorname{Sin}[w]}{9+6 \operatorname{Cos}[w]} \text { and } \\
W_{(W E N O)}=\frac{1}{30}(i(-10+15 \operatorname{Cos}[w]-6 \operatorname{Cos}[2 w]+\operatorname{Cos}[3 w])+45 \operatorname{Sin}[w]-9 \operatorname{Sin}[2 w]+\operatorname{Sin}[3 w])
\end{gathered}
$$

The dispersion errors of WUCS, WCS, and the WENO scheme are

$$
\begin{gathered}
\operatorname{Re}\left(w_{(W U C S)}\right)=\operatorname{Re}\left(\frac{2 i+34 i \operatorname{Cos}[w]-34 i \operatorname{Cos}[2 w]-2 i \operatorname{Cos}[3 w]+270 \operatorname{Sin}[w]-27 \operatorname{Sin}[2 w]-2 \operatorname{Sin}[3 w]}{6(26+12 \operatorname{Cos}[w]-3 \operatorname{Cos}[2 w]+4 i \operatorname{Sin}[w]+3 i \operatorname{Sin}[2 w])}\right) \\
\begin{aligned}
\operatorname{Re}\left(w_{(\mathrm{WCS})}\right) & =\operatorname{Re}\left(\frac{(14+\operatorname{Cos}[w]) \operatorname{Sin}[w]}{9+6 \operatorname{Cos}[w]}\right) \\
& =\frac{(14+\operatorname{Cos}[w]) \operatorname{Sin}[w]}{9+6 \operatorname{Cos}[w]}, \text { and } \\
\operatorname{Re}\left(W_{(W E N O)}\right)= & \operatorname{Re}\left(\frac{1}{30}(i(-10+15 \operatorname{Cos}[w]-6 \operatorname{Cos}[2 w]+\operatorname{Cos}[3 w])+45 \operatorname{Sin}[w]-9 \operatorname{Sin}[2 w]+\operatorname{Sin}[3 w])\right) \\
= & \frac{1}{30}(45 \operatorname{Sin}[w]-9 \operatorname{Sin}[2 w]+\operatorname{Sin}[3 w]) \\
= & \frac{3}{2} \operatorname{Sin}[w]-\frac{3}{10} \operatorname{Sin}[2 w]+\frac{1}{30} \operatorname{Sin}[3 w]
\end{aligned}
\end{gathered}
$$

Figure 3.11 shows that WUCS has small dispersion errors and achieves the highest resolution compared to WCS and the WENO scheme.

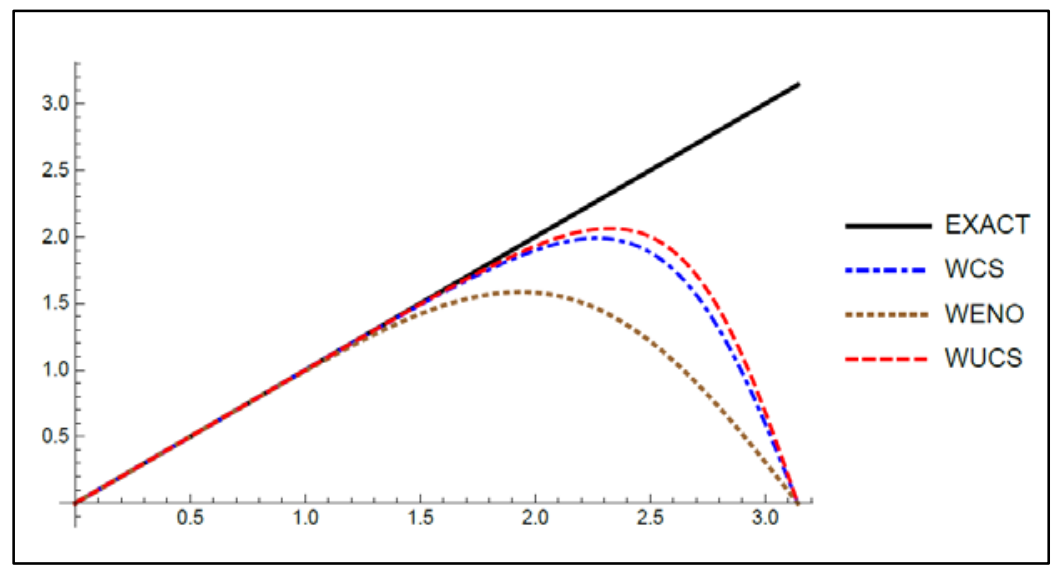

Figure 3.11. The dispersion in WUCS, WCS, and the WENO scheme. 
The dissipation errors are

$$
\begin{gathered}
-\operatorname{Im}\left(w_{(W U C S)}\right)=-\operatorname{Im}\left(\frac{2 i+34 i \operatorname{Cos}[w]-34 i \operatorname{Cos}[2 w]-2 i \operatorname{Cos}[3 w]+270 \operatorname{Sin}[w]-27 \operatorname{Sin}[2 w]-2 \operatorname{Sin}[3 w]}{6(26+12 \operatorname{Cos}[w]-3 \operatorname{Cos}[2 w]+4 i \operatorname{Sin}[w]+3 i \operatorname{Sin}[2 w])}\right) \\
-\operatorname{Im}\left(w_{(W C S)}\right)=-\operatorname{Im}\left(\frac{(14+\operatorname{Cos}[w]) \operatorname{Sin}[w]}{9+6 \operatorname{Cos}[w]}\right)=0, \text { and } \\
-\operatorname{Im}\left(W_{(W E N O)}\right)=-\operatorname{Im}\left(\frac{1}{30}(i(-10+15 \operatorname{Cos}[w]-6 \operatorname{Cos}[2 w]+\operatorname{Cos}[3 w])+45 \operatorname{Sin}[w]-9 \operatorname{Sin}[2 w]+\operatorname{Sin}[3 w])\right) \\
=-\left(\frac{1}{30}(-10+15 \operatorname{Cos}[w]-6 \operatorname{Cos}[2 w]+\operatorname{Cos}[3 w])\right) \\
=\frac{1}{3}-\frac{1}{2} \operatorname{Cos}[w]+\frac{1}{5} \operatorname{Cos}[2 w]-\frac{1}{30} \operatorname{Cos}[3 w]
\end{gathered}
$$

Similar to the analysis of the center stencils, Figure 3.12 demonstrates that WCS has no dissipation errors while both the WENO scheme and WUCS are positively dissipative. Also, the dissipation errors in the WENO scheme are the highest among all the three schemes.

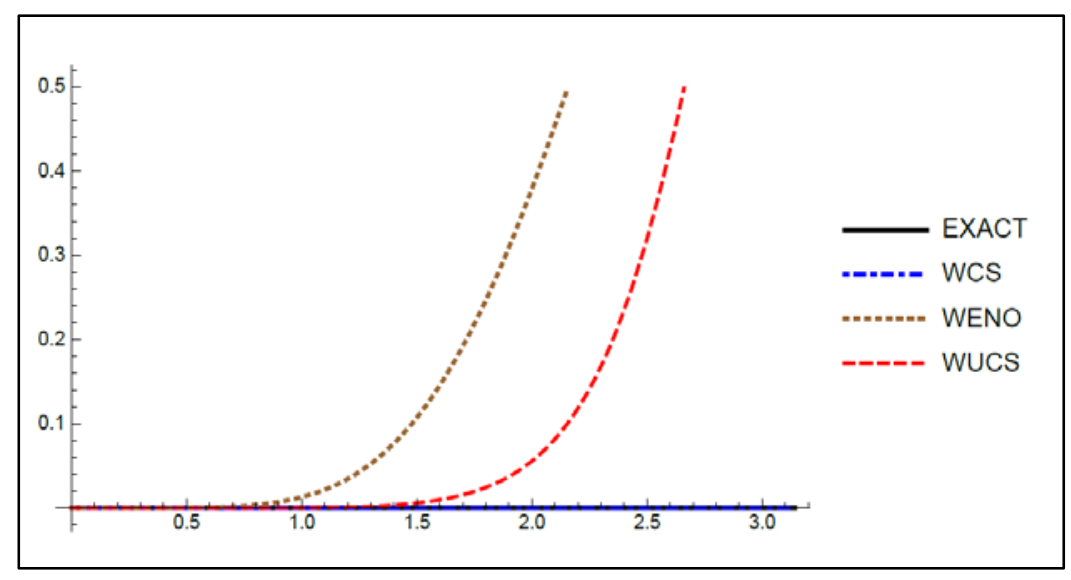

Figure 3.12. The dissipation in WUCS, WCS, and the WENO scheme. 


\subsection{Decoupling the System of WUCS}

WCS has a global dependency problem because it uses all grid points including the downstream points to get the derivative near shocks. WUCS applies the technique of decoupling the system in shock areas to change the global dependence into an upwinding dependence. To explain the idea of this section, systems or matrices from WCS and WUCS are illustrated. From (3.31), the final scheme of WCS is

$$
\frac{1}{3} H^{\prime}{ }_{j-\frac{1}{2}}+H^{\prime}{ }_{j+\frac{1}{2}}+\frac{1}{3} H^{\prime}{ }_{j+\frac{3}{2}} \approx \frac{1}{h}\left(\frac{23}{36} H_{j-\frac{3}{2}}-\frac{7}{9} H_{j-\frac{1}{2}}-\frac{1}{9} H_{j+\frac{3}{2}}+\frac{1}{36} H_{j+\frac{5}{2}}\right)
$$

Hence, (3.31) can be written in a matrix form as follows:

$$
A H^{\prime}=\frac{1}{h} B H
$$

where $\quad H=\left[\begin{array}{cccccccccccc}H_{\frac{1}{2}} & H_{1+\frac{1}{2}} & H_{2+\frac{1}{2}} & \ldots & \ldots & \ldots & \ldots & \ldots & \ldots & \ldots & H_{n-1+\frac{1}{2}} & H_{n+\frac{1}{2}}\end{array}\right]^{T}$, $H^{\prime}=\left[\begin{array}{rrrrrrrrrrrr}H^{\prime}{ }_{\frac{1}{2}}^{\prime} & H^{\prime}{ }_{1+\frac{1}{2}} & H^{\prime}{ }_{2+\frac{1}{2}} & \ldots & \ldots & \ldots & \ldots & \ldots & \ldots & \ldots & H^{\prime}{ }_{n-\frac{1}{2}} & H^{\prime}{ }_{n+\frac{1}{2}}\end{array}\right]^{T}$, and

$\mathrm{A}$ is the derivative matrix of the WCS. A is a tri-diagonal matrix, which can be written as follows:

$$
A=\left[\begin{array}{ccccccccccccc}
b_{0} & c_{0} & & & & & & & & & & & \\
a & b & c & & & & & & & & & & \\
& a & b & c & & & & & & & & & \\
& & a & b & c & & & & & & & & \\
& & & \ddots & \ddots & \ddots & & & & & & & \\
& & & & a & b & c & & & & & & \\
& & & & & a & b & c & & & & & \\
& & & & & & a & b & c & & & & \\
& & & & & & & a & b & c & & & \\
& & & & & & & & \ddots & \ddots & \ddots & & \\
& & & & & & & & & a & b & c & \\
& & & & & & & & & & a & b & c \\
& & & & & & & & & & & a_{n} & b_{n}
\end{array}\right]
$$

where $a=\frac{1}{3}, b=1$, and $c=\frac{1}{3}$. 
Also, the matrix B can be written as:

$$
B=\left[\begin{array}{ccccccccccccc}
t_{0} & r_{0} & d_{0} & & & & & & & & & \\
s_{1} & t_{1} & r_{1} & d_{1} & & & & & & & & \\
e & s & t & r & d & & & & & & & \\
& e & s & t & r & d & & & & & & \\
& & \ddots & \ddots & \ddots & \ddots & \ddots & & & & & \\
& & & e & s & t & r & d & & & & \\
& & & & e & s & t & r & d & & & \\
& & & & & e & s & t & r & d & & \\
& & & & & & \ddots & \ddots & \ddots & \ddots & \ddots & \\
& & & & & & & e & s & t & r & d \\
& & & & & & & & e & s_{m} & t_{m} & r_{m} \\
& & & & & & & & & e_{n} & s_{n} & t_{n}
\end{array}\right]
$$

where $e=\frac{23}{36}, s=-\frac{7}{9}, t=0, r=-\frac{1}{9}$, and $d=\frac{1}{36}$.

Near shock areas, the bias weights from the WENO scheme (3.22) are used with $\mathrm{p}=1$.

Let us assume the shock location is detected as shown below:

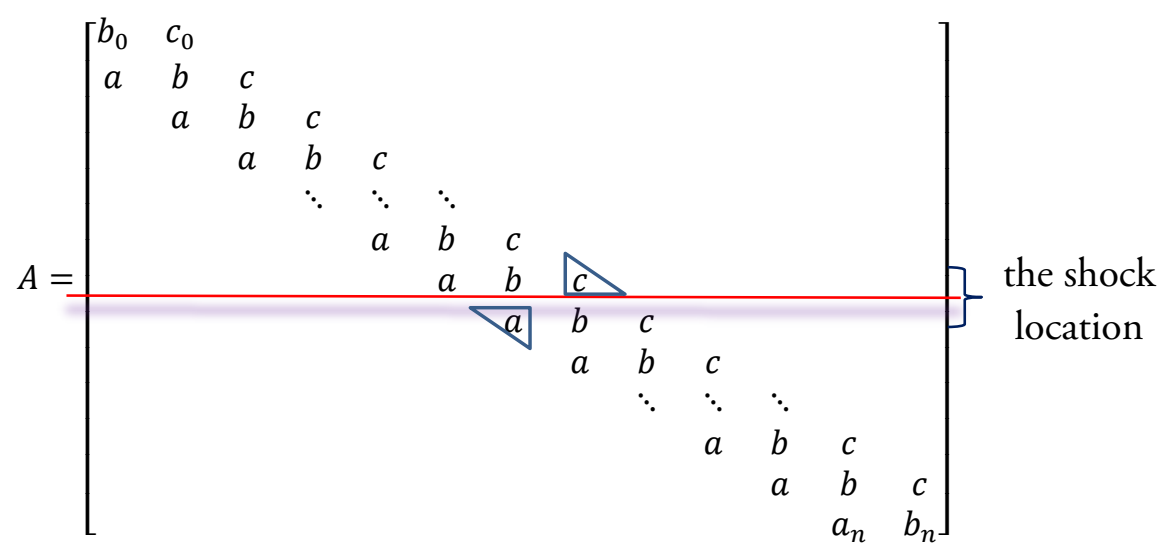

WCS minimizes the influence of a shock-containing candidate stencil by assigning a smaller weight. However, WCS is global dependent resulting from the use of all grid points including the downstream points (inside the triangles) to get the derivative, and such usage is prohibited for the shock case. 
As illustrated in (3.38), the $7^{\text {th }}$ order WUCS can be used in smooth areas, and it is given as follows:

$$
\begin{aligned}
\frac{3}{13} \hat{f}_{j-\frac{3}{2}}-\frac{4}{13} \hat{f}_{j-\frac{1}{2}}-2 \hat{f}_{j+\frac{1}{2}}-\frac{8}{13} \hat{f}_{j+\frac{3}{2}} & \\
& =\frac{1}{39} f_{j-2}+\frac{5}{12} f_{j-1}-\frac{239}{156} f_{j}-\frac{81}{52} f_{j+1}-\frac{7}{156} f_{j+2}
\end{aligned}
$$

The WUCS can be written in a matrix form as

$$
A \widehat{F}=G
$$

where $\mathrm{A}$ is the derivative matrix of the WUCS, which is a penta-diagonal matrix that can be written as follows:

$$
A=\left[\begin{array}{ccccccccccccc}
1 & 0 & 0 & & & & & & & & & \\
0 & 1 & 0 & 0 & & & & & & & & \\
0 & 0 & 1 & 0 & 0 & & & & & & & \\
& a & b & c & d & e & & & & & & \\
& & \ddots & \ddots & \ddots & \ddots & \ddots & & & & & \\
& & & a & b & c & d & e & & & & \\
& & & & a & b & c & d & e & & & \\
& & & & & a & b & c & d & e & & \\
& & & & & & \ddots & \ddots & \ddots & \ddots & \ddots & \\
& & & & & & & a & b & c & d & e \\
& & & & & & & & 0 & 0 & 1 & 0 \\
& & & & & & & & & 0 & 0 & 1
\end{array}\right],
$$

where $a=\frac{3}{13}, b=-\frac{4}{13}, c=-2, d=-\frac{8}{13}$, and $e=0$.

$\widehat{F}=\left[\begin{array}{llllllllllll}\hat{f}_{\frac{1}{2}} & \hat{f}_{1+\frac{1}{2}} & \hat{f}_{2+\frac{1}{2}} & \ldots & \ldots & \ldots & \ldots & \ldots & \ldots & \ldots & \hat{f}_{n-\frac{1}{2}} & \hat{f}_{n+\frac{1}{2}}\end{array}\right]^{T}$, and

$G=B F=\left[\begin{array}{llllllllllll}g_{0} & g_{1} & g_{2} & \cdots & \cdots & \cdots & \ldots & \ldots & \ldots & \ldots & g_{n-1} & g_{n}\end{array}\right]^{T}$

where $F=\left[\begin{array}{llllllllllll}f_{0} & f_{1} & f_{2} & \ldots & \ldots & \ldots & \ldots & \ldots & \ldots & \ldots & f_{n-1} & f_{n}\end{array}\right]^{T}$ and 


$$
B=\left[\begin{array}{ccccccccccccc}
a_{1} & a_{2} & a_{3} & a_{4} & a_{5} & a_{6} & a_{7} & & & & & & \\
b_{1} & b_{2} & b_{3} & b_{4} & b_{5} & b_{6} & b_{7} & & & & & \\
c_{4} & c_{4} & c_{4} & c_{4} & c_{4} & c_{4} & c_{4} & & & & & \\
r & s & t & u & v & & & & & & & \\
& \ddots & \ddots & \ddots & \ddots & \ddots & & & & & & \\
& & r & s & t & u & v & & & & & \\
& & & r & s & t & u & v & & & & & \\
& & & & r & s & t & u & v & & & & \\
& & & & & r & s & t & u & v & & & \\
& & & & & & \ddots & \ddots & \ddots & \ddots & \ddots & & \\
& & & & & & & r & s & t & u & v & \\
& & & & & & d_{1} & d_{2} & d_{3} & d_{4} & d_{5} & d_{6} & d_{7} \\
& & & & & & e_{1} & e_{2} & e_{3} & e_{4} & e_{5} & e_{6} & e_{7}
\end{array}\right]
$$

where

$$
\begin{aligned}
& r=\frac{1}{39}, s=\frac{5}{12}, t=-\frac{239}{156}, u=-\frac{81}{52}, v=-\frac{7}{156}, \\
& a_{1}=\frac{363}{140}, a_{2}=-\frac{617}{140}, a_{3}=\frac{853}{140}, a_{4}=-\frac{2341}{420}, a_{5}=\frac{667}{210}, a_{6}=-\frac{43}{42}, a_{7}=\frac{1}{7}, \\
& b_{1}=\frac{1}{7}, b_{2}=\frac{223}{140}, b_{3}=-\frac{197}{140}, b_{4}=\frac{153}{140}, b_{5}=-\frac{241}{420}, b_{6}=\frac{37}{210}, b_{7}=-\frac{1}{42}, \\
& c_{1}=-\frac{1}{42}, c_{2}=\frac{13}{42}, c_{3}=\frac{153}{140}, c_{4}=-\frac{241}{420}, c_{5}=\frac{109}{140}, c_{6}=-\frac{31}{420}, c_{7}=\frac{1}{105}, \\
& d_{1}=-\frac{1}{42}, d_{2}=\frac{37}{210}, d_{3}=-\frac{241}{420}, d_{4}=-\frac{153}{140}, d_{5}=-\frac{197}{140}, d_{6}=\frac{223}{140}, d_{7}=\frac{1}{7}, \\
& e_{1}=\frac{1}{7}, e_{2}=-\frac{43}{42}, e_{3}=\frac{667}{210}, e_{4}=-\frac{2341}{420}, e_{5}=\frac{853}{140}, e_{6}=-\frac{617}{140}, \text { and } e_{7}=\frac{363}{140} .
\end{aligned}
$$

Suppose the shock is located between $f_{\mathrm{k}}$ and $f_{\mathrm{k}+1}$ as shown below:

$$
G=B F
$$




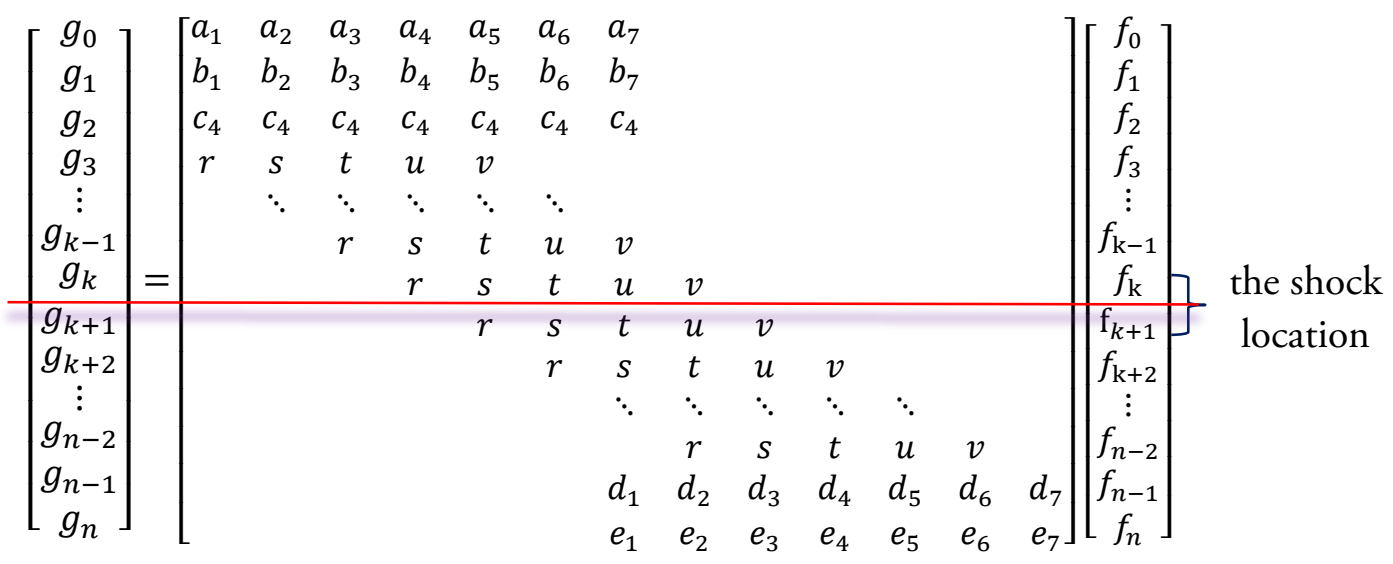

The location of the shock in the derivative matrix A will be located as follows:

$$
A \hat{F}=G
$$

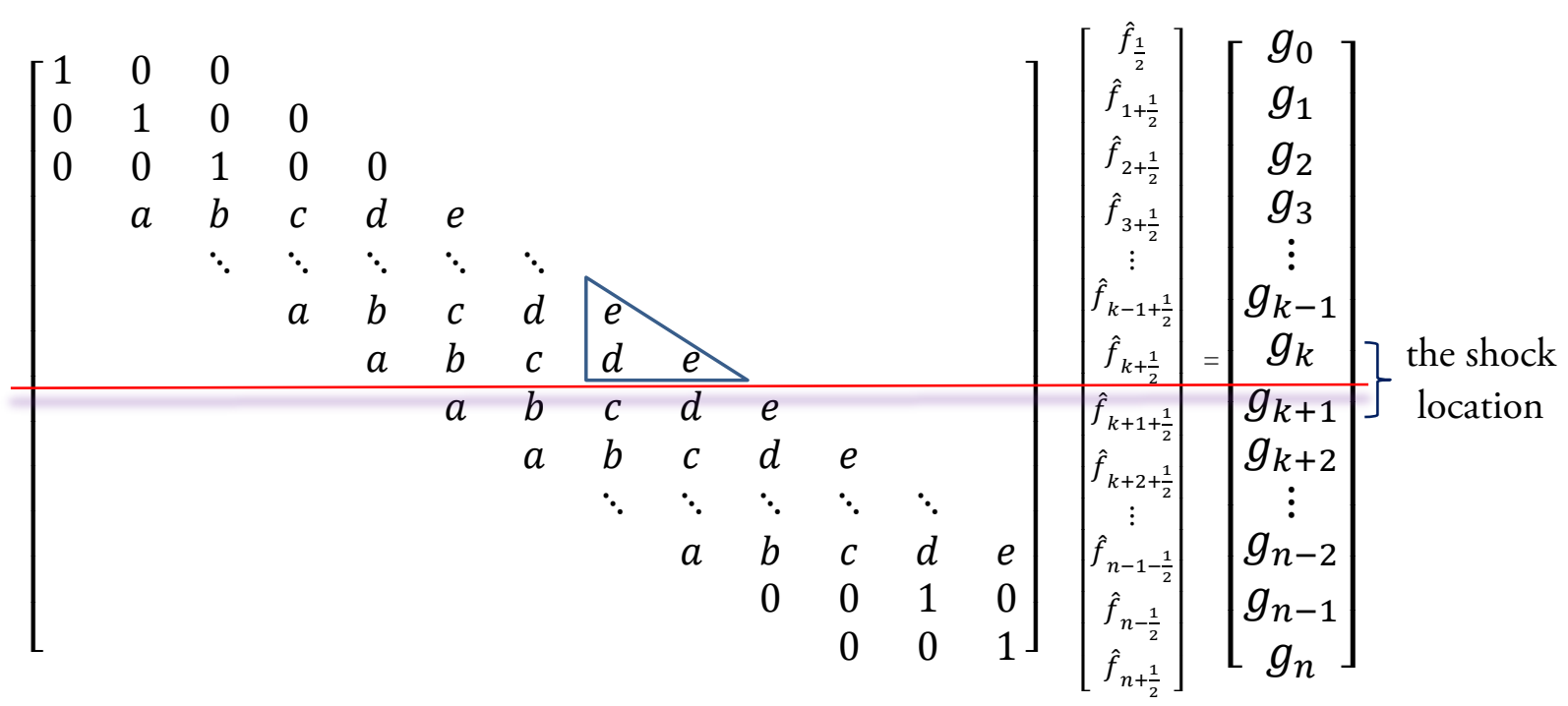

The elements inside the triangle lead to the global dependency problem because of the downstream points $g_{\mathrm{k}+1}$ and $g_{\mathrm{k}+2}$, which means using $f_{\mathrm{k}+1}$ and $f_{\mathrm{k}+2}$ to get the derivative of $f_{\mathrm{k}}$. This problem is avoided in WUCS by decoupling the derivative matrix into two submatrices as shown below. 


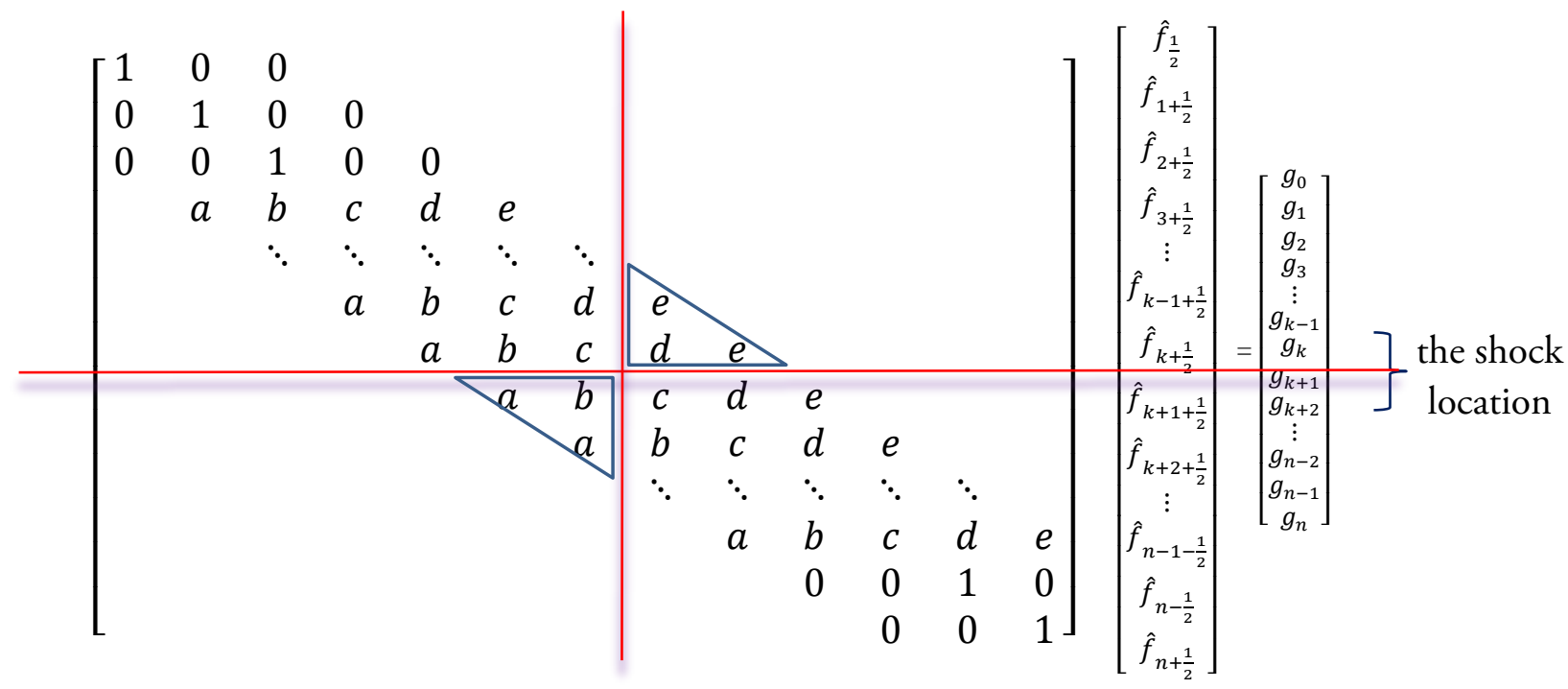

The shock points are treated as boundary points, so the elements inside the two triangles above become zeros. The resulting subsystems or submatrices are $\left[\begin{array}{ccccccccc}1 & 0 & 0 & & & & & & \\ 0 & 1 & 0 & 0 & & & & & \\ 0 & 0 & 1 & 0 & 0 & & & & \\ & a & b & c & d & e & & & \\ & & \ddots & \ddots & \ddots & \ddots & \ddots & & \\ & & & \ddots & \ddots & \ddots & \ddots & \ddots & \\ & & & & a & b & c & d & e \\ & & & & & & 0 & 1 & 0 \\ \hat{f}_{k-2+\frac{1}{2}} \\ \hat{f}_{k-1+\frac{1}{2}} \\ \hat{f}_{k+\frac{1}{2}}\end{array}\right]=\left[\begin{array}{c}\hat{f}_{\frac{1}{2}} \\ \hat{f}_{1+\frac{1}{2}} \\ \hat{f}_{2+\frac{1}{2}} \\ \hat{f}_{3+\frac{1}{2}} \\ \vdots \\ g_{k-2} \\ g_{k-1} \\ g_{k}\end{array}\right]=\left[\begin{array}{c}g_{0} \\ g_{1} \\ g_{2} \\ g_{3} \\ \vdots \\ \end{array}\right.$ $\left[\begin{array}{ccccccccc}1 & 0 & 0 & & & & & & \\ 0 & 1 & 0 & 0 & & & & & \\ 0 & 0 & 1 & 0 & 0 & & & & \\ & a & b & c & d & e & & & \\ & & \ddots & \ddots & \ddots & \ddots & \ddots & & \\ & & & \ddots & \ddots & \ddots & \ddots & \ddots & \\ & & & & a & b & c & d & e \\ & & & & & & 0 & 1 & 0 \\ \hat{f}_{k+2+\frac{1}{2}} \\ \hat{f}_{k+2+\frac{1}{2}} \\ \vdots \\ \vdots \\ \hat{f}_{n-1-\frac{1}{2}} \\ \hat{f}_{n-\frac{1}{2}} \\ \hat{f}_{n+\frac{1}{2}}\end{array}\right]=\left[\begin{array}{c}\hat{f}_{k+1+\frac{1}{2}} \\ \hat{f}_{k+1} \\ g_{k+2} \\ g_{k+3} \\ g_{k+4} \\ \vdots \\ \vdots \\ g_{n-2} \\ g_{n-1} \\ g_{n}\end{array}\right]$. 
Also, the right hand side of the system will be decoupled into the following subsystems:

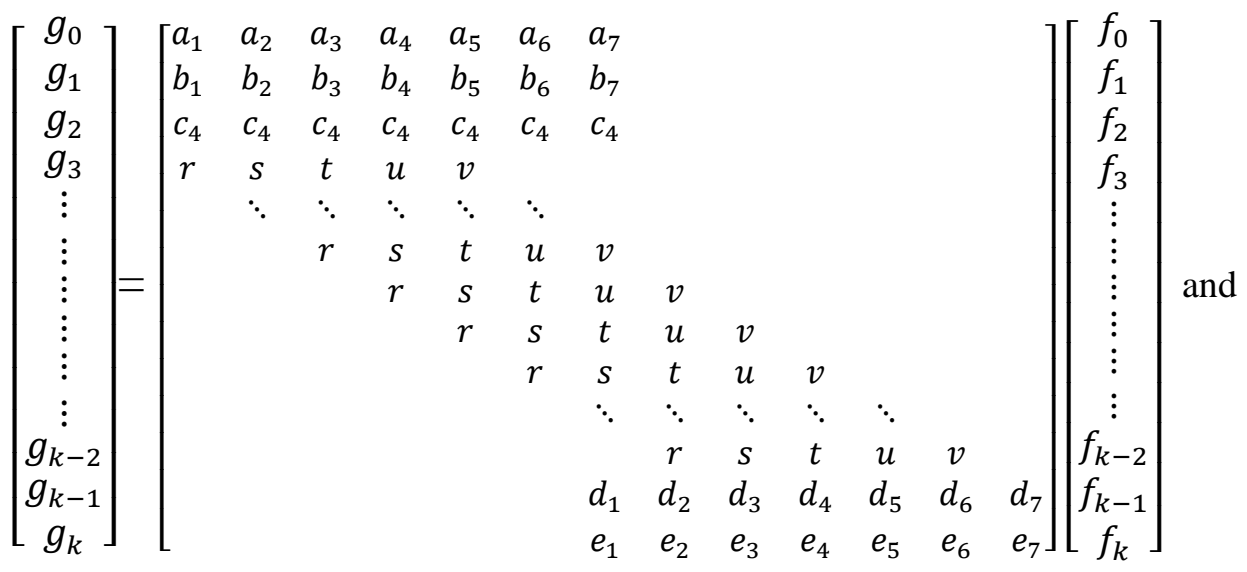

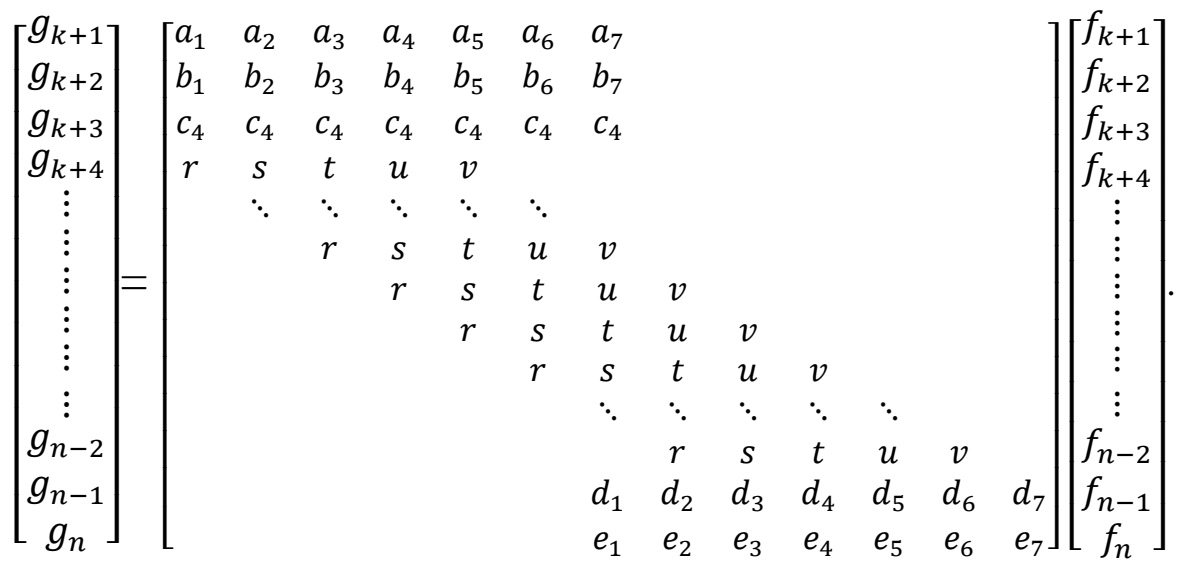




\subsection{Conclusion}

In this chapter, the Weighted Upwinding Compact Scheme (WUCS), which belongs to the family of finite difference schemes, is proposed. Based on the analysis of the dissipation and dispersion errors, the upwinding candidates are constructed in a way such that the left and central candidates have a non-negative dissipation. In addition, the analysis shows that WUCS has small dispersion errors and achieves the highest resolution compared to WCS and the WENO scheme.

Furthermore, the global dependency problem, using all grid points including the downstream points to get the derivative, is prohibited for the shock case, so it is avoided with WUCS by decoupling the system into two subsystems. 


\section{CHAPTER 4}

\section{NUMERICAL RESULTS}

Runge-Kutta scheme (Shu et al., 1988) is used for time integration, and WUCS is used to find the derivative in the $\mathrm{x}$ direction for the following examples.

\subsection{Convection Equation}

The one-dimensional convection equation

$$
u_{t}+u_{x}=0, \quad a<x<b
$$

is solved using WUCS at time tk with different initial functions

$$
u(x, 0)=u_{0}(x)
$$

Two types of initial functions are used, smooth and non-smooth. For the smooth ones, WUCS is used directly to solve the equation as in section 4.1.1. Also, high frequency examples are solved to indicate the scheme has a $7^{\text {th }}$ order of accuracy with high resolution as in section 4.1.2. For the non-smooth functions, the proposed scheme is applied in two ways. The first way is using the idea of the non-linear weight from the WENO scheme by giving very small weights for the candidates that have discontinuities as in section 4.1.3. Secondly, the system is decupled into subsystems and solving these subsystems using WUCS. In other words, the derivative matrix is decoupled into submatrices according to shock locations as in section 4.1.4. 
4.1.1 $u(x, 0)=u_{0}(x), \quad 0<x<2$

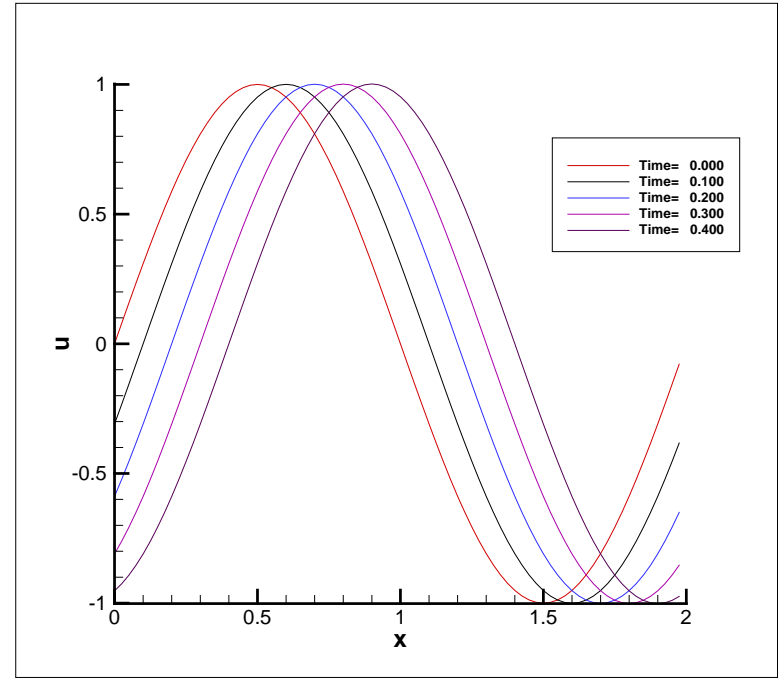

Figure 4.1. The numerical solution from WUCS when $u_{0}(x)=\sin (\pi x)$ at different time steps.

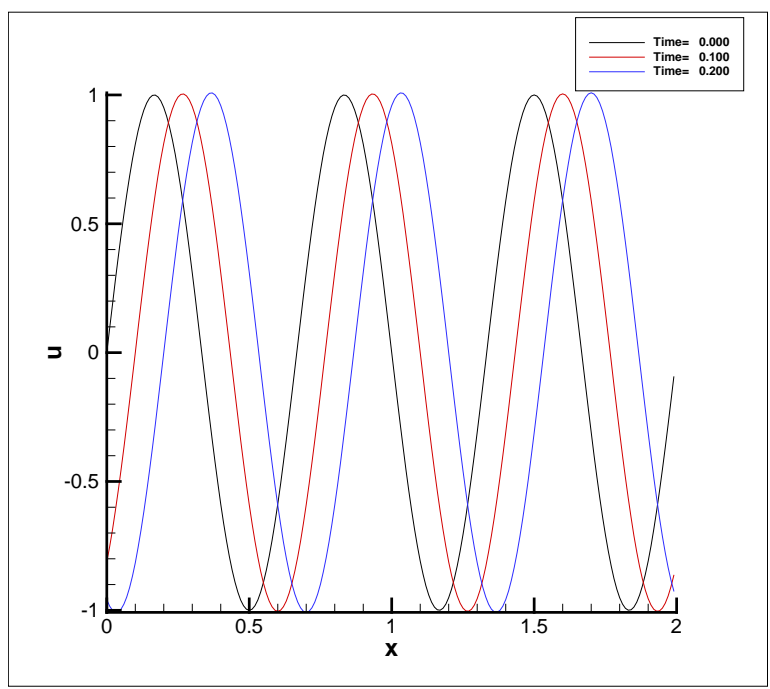

Figure 4.3. The numerical solution from WUCS when $u_{0}(x)=\sin (3 \pi x)$ at different time steps.

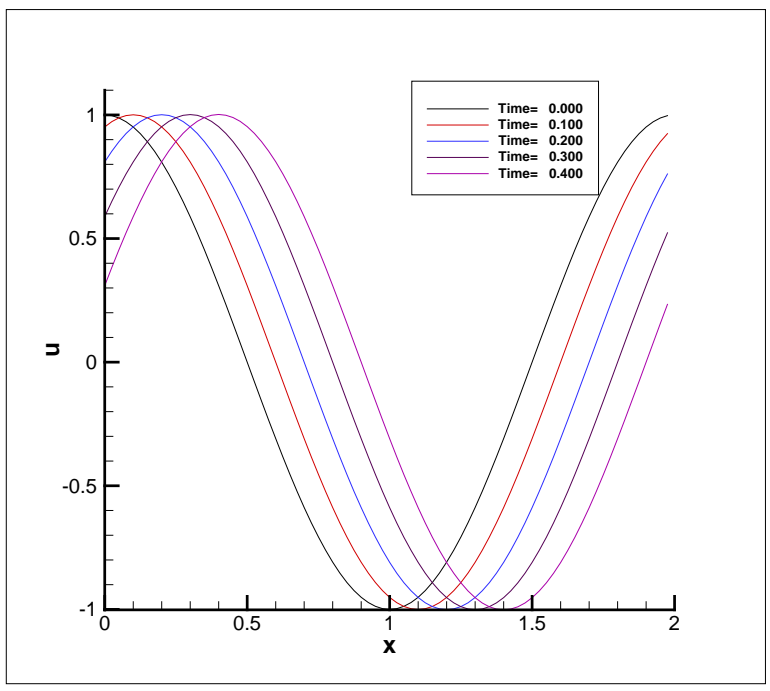

Figure 4.2. The numerical solution from WUCS when $u_{0}(x)=\cos (\pi x)$ at different time steps.

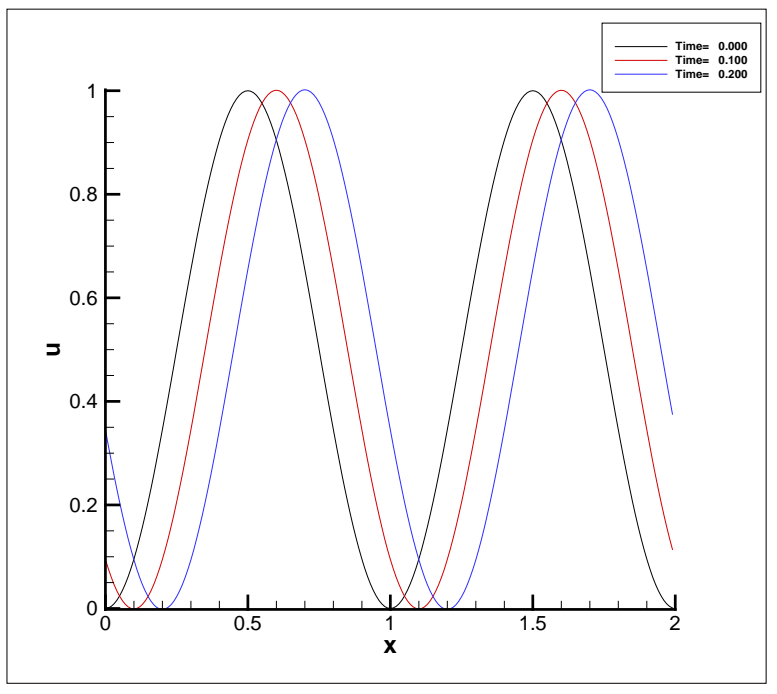

Figure 4.4. The numerical solution from WUCS when $u_{0}(x)=(\sin (\pi x))^{2}$ at different time steps. 
4.1.2 $u(x, 0)=u_{0}(x),-\pi<x<\pi$

Table 4.1. Errors of the numerical solution from WUCS when $u_{0}(x)=\sin (x)$ at $t_{k}=1$

\begin{tabular}{||c|cccccccccc||}
\hline \hline $\mathbf{N}$ & $\begin{array}{c}\mathbf{L}_{\infty} \\
\text { ERROR }\end{array}$ & $\mathbf{H} / \mathbf{2 H}$ & $\begin{array}{c}\mathbf{L}_{\infty} \\
\text { ORDER }\end{array}$ & $\begin{array}{c}\mathbf{L}_{1} \\
\text { ERROR }\end{array}$ & H/2H & $\begin{array}{c}\mathbf{L}_{1} \\
\text { ORDER }\end{array}$ & $\begin{array}{c}\mathbf{L}_{2} \\
\text { ERROR }\end{array}$ & H/2H & $\begin{array}{c}\mathbf{L}_{2} \\
\text { ORDER }\end{array}$ \\
$\mathbf{1 0}$ & $1.36 \mathrm{E}-05$ & & & $5.20 \mathrm{E}-05$ & & & $2.39 \mathrm{E}-05$ & & \\
$\mathbf{2 0}$ & $1.11 \mathrm{E}-07$ & $1.23 \mathrm{E}+02$ & $\mathbf{6 . 9 4}$ & $4.39 \mathrm{E}-07$ & $1.19 \mathrm{E}+02$ & $\mathbf{6 . 8 9}$ & $1.97 \mathrm{E}-07$ & $1.22 \mathrm{E}+02$ & $\mathbf{6 . 9 3}$ \\
$\mathbf{4 0}$ & $8.78 \mathrm{E}-10$ & $1.26 \mathrm{E}+02$ & $\mathbf{6 . 9 8}$ & $3.50 \mathrm{E}-09$ & $1.25 \mathrm{E}+02$ & $\mathbf{6 . 9 7}$ & $1.56 \mathrm{E}-09$ & $1.26 \mathrm{E}+02$ & $\mathbf{6 . 9 8}$ \\
$\mathbf{8 0}$ & $6.89 \mathrm{E}-12$ & $1.28 \mathrm{E}+02$ & $\mathbf{6 . 9 9}$ & $2.75 \mathrm{E}-11$ & $1.27 \mathrm{E}+02$ & $\mathbf{6 . 9 9}$ & $1.22 \mathrm{E}-11$ & $1.28 \mathrm{E}+02$ & $\mathbf{7 . 0 0}$ \\
$\mathbf{1 6 0}$ & $7.34 \mathrm{E}-14$ & $9.38 \mathrm{E}+01$ & $\mathbf{6 . 5 5}$ & $2.18 \mathrm{E}-13$ & $1.26 \mathrm{E}+02$ & $\mathbf{6 . 9 8}$ & $9.78 \mathrm{E}-14$ & $1.25 \mathrm{E}+02$ & $\mathbf{6 . 9 6}$ \\
\hline
\end{tabular}

Table 4.2. Errors of the numerical solution from WUCS when $u_{0}(x)=\sin (10 x)$ at $t_{k}=1$

\begin{tabular}{||c|ccccccccccc||}
\hline \hline $\mathbf{N}$ & $\begin{array}{c}\text { L } \infty \\
\text { ERROR }\end{array}$ & H/2H & $\begin{array}{c}\text { L } \infty \\
\text { ORDER }\end{array}$ & $\begin{array}{c}\text { L1 } \\
\text { ERROR }\end{array}$ & H/2H & $\begin{array}{c}\text { L1 } \\
\text { ORDER }\end{array}$ & $\begin{array}{c}\text { L2 } \\
\text { ERROR }\end{array}$ & H/2H & $\begin{array}{c}\text { L2 } \\
\text { ORDER }\end{array}$ \\
\hline $\mathbf{4 0}$ & $5.58 \mathrm{E}-02$ & & & $3.38 \mathrm{E}-01$ & & & $1.36 \mathrm{E}-01$ & & \\
$\mathbf{8 0}$ & $6.14 \mathrm{E}-04$ & $9.08 \mathrm{E}+01$ & $\mathbf{6 . 5}$ & $2.59 \mathrm{E}-03$ & $1.30 \mathrm{E}+02$ & $\mathbf{7 . 0 3}$ & $1.13 \mathrm{E}-03$ & $1.21 \mathrm{E}+02$ & $\mathbf{6 . 9 1}$ \\
$\mathbf{1 6 0}$ & $5.23 \mathrm{E}-06$ & $1.18 \mathrm{E}+02$ & $\mathbf{6 . 8 8}$ & $2.11 \mathrm{E}-05$ & $1.23 \mathrm{E}+02$ & $\mathbf{6 . 9 4}$ & $9.33 \mathrm{E}-06$ & $1.21 \mathrm{E}+02$ & $\mathbf{6 . 9 2}$ \\
$\mathbf{3 2 0}$ & $4.18 \mathrm{E}-08$ & $1.25 \mathrm{E}+02$ & $\mathbf{6 . 9 7}$ & $1.67 \mathrm{E}-07$ & $1.26 \mathrm{E}+02$ & $\mathbf{6 . 9 8}$ & $7.42 \mathrm{E}-08$ & $1.26 \mathrm{E}+02$ & $\mathbf{6 . 9 7}$ \\
$\mathbf{6 4 0}$ & $3.28 \mathrm{E}-10$ & $1.27 \mathrm{E}+02$ & $\mathbf{6 . 9 9}$ & $1.31 \mathrm{E}-09$ & $1.27 \mathrm{E}+02$ & $\mathbf{6 . 9 9}$ & $5.82 \mathrm{E}-10$ & $1.27 \mathrm{E}+02$ & $\mathbf{6 . 9 9}$ \\
\hline $\mathbf{1 2 8 0}$ & $2.88 \mathrm{E}-12$ & $1.14 \mathrm{E}+02$ & $\mathbf{6 . 8 3}$ & $1.05 \mathrm{E}-11$ & $1.25 \mathrm{E}+02$ & $\mathbf{6 . 9 7}$ & $4.67 \mathrm{E}-12$ & $1.25 \mathrm{E}+02$ & $\mathbf{6 . 9 6}$ \\
\hline
\end{tabular}


Table 4.3. Errors of the numerical solution from WUCS when $u_{0}(x)=\sin (20 x)$ at $t_{k}=1$

\begin{tabular}{||c|cccccccccc||}
\hline \hline $\mathbf{N}$ & $\begin{array}{c}\text { L } \infty \\
\text { ERROR }\end{array}$ & H/2H & $\begin{array}{c}\text { L } \infty \\
\text { ORDER }\end{array}$ & $\begin{array}{c}\text { L1 } \\
\text { ERROR }\end{array}$ & H/2H & $\begin{array}{c}\text { L1 } \\
\text { ORDER }\end{array}$ & $\begin{array}{c}\text { L2 } \\
\text { ERROR }\end{array}$ & H/2H & $\begin{array}{c}\text { L2 } \\
\text { ORDER }\end{array}$ \\
\hline $\mathbf{8 0}$ & $1.12 \mathrm{E}-01$ & & & $6.84 \mathrm{E}-01$ & & & $2.75 \mathrm{E}-01$ & & \\
$\mathbf{1 6 0}$ & $1.23 \mathrm{E}-03$ & $9.08 \mathrm{E}+01$ & $\mathbf{6 . 5}$ & $5.20 \mathrm{E}-03$ & $1.32 \mathrm{E}+02$ & $\mathbf{7 . 0 4}$ & $2.27 \mathrm{E}-03$ & $1.21 \mathrm{E}+02$ & $\mathbf{6 . 9 2}$ \\
$\mathbf{3 2 0}$ & $1.05 \mathrm{E}-05$ & $1.18 \mathrm{E}+02$ & $\mathbf{6 . 8 8}$ & $4.22 \mathrm{E}-05$ & $1.23 \mathrm{E}+02$ & $\mathbf{6 . 9 4}$ & $1.87 \mathrm{E}-05$ & $1.21 \mathrm{E}+02$ & $\mathbf{6 . 9 2}$ \\
$\mathbf{6 4 0}$ & $8.35 \mathrm{E}-08$ & $1.25 \mathrm{E}+02$ & $\mathbf{6 . 9 7}$ & $3.35 \mathrm{E}-07$ & $1.26 \mathrm{E}+02$ & $\mathbf{6 . 9 8}$ & $1.48 \mathrm{E}-07$ & $1.26 \mathrm{E}+02$ & $\mathbf{6 . 9 8}$ \\
$\mathbf{1 2 8 0}$ & $6.57 \mathrm{E}-10$ & $1.27 \mathrm{E}+02$ & $\mathbf{6 . 9 9}$ & $2.63 \mathrm{E}-09$ & $1.27 \mathrm{E}+02$ & $\mathbf{6 . 9 9}$ & $1.16 \mathrm{E}-09$ & $1.27 \mathrm{E}+02$ & $\mathbf{6 . 9 9}$ \\
\hline
\end{tabular}

Table 4.4. Errors of the numerical solution from WUCS when $u_{0}(x)=\sin (40 x)$ at $t_{k}=1$

\begin{tabular}{||c|cccccccccc||}
\hline \hline $\mathbf{N}$ & $\begin{array}{c}\text { L } \infty \\
\text { ERROR }\end{array}$ & H/2H & $\begin{array}{c}\text { L } \infty \\
\text { ORDER }\end{array}$ & $\begin{array}{c}\text { L1 } \\
\text { ERROR }\end{array}$ & H/2H & $\begin{array}{c}\text { L1 } \\
\text { ORDER }\end{array}$ & $\begin{array}{c}\text { L2 } \\
\text { ERROR }\end{array}$ & H/2H & $\begin{array}{c}\text { L2 } \\
\text { ORDER }\end{array}$ \\
\hline $\mathbf{1 6 0}$ & $2.23 \mathrm{E}-01$ & & & $1.38 \mathrm{E}+00$ & & & $5.51 \mathrm{E}-01$ & & \\
$\mathbf{3 2 0}$ & $2.46 \mathrm{E}-03$ & $9.08 \mathrm{E}+01$ & $\mathbf{6 . 5}$ & $1.04 \mathrm{E}-02$ & $1.32 \mathrm{E}+02$ & $\mathbf{7 . 0 4}$ & $4.53 \mathrm{E}-03$ & $1.21 \mathrm{E}+02$ & $\mathbf{6 . 9 2}$ \\
$\mathbf{6 4 0}$ & $2.09 \mathrm{E}-05$ & $1.18 \mathrm{E}+02$ & $\mathbf{6 . 8 8}$ & $8.45 \mathrm{E}-05$ & $1.23 \mathrm{E}+02$ & $\mathbf{6 . 9 5}$ & $3.73 \mathrm{E}-05$ & $1.22 \mathrm{E}+02$ & $\mathbf{6 . 9 2}$ \\
$\mathbf{1 2 8 0}$ & $1.67 \mathrm{E}-07$ & $1.25 \mathrm{E}+02$ & $\mathbf{6 . 9 7}$ & $6.70 \mathrm{E}-07$ & $1.26 \mathrm{E}+02$ & $\mathbf{6 . 9 8}$ & $2.97 \mathrm{E}-07$ & $1.26 \mathrm{E}+02$ & $\mathbf{6 . 9 8}$ \\
\hline $\mathbf{2 5 6 0}$ & $1.31 \mathrm{E}-09$ & $1.27 \mathrm{E}+02$ & $\mathbf{6 . 9 9}$ & $5.25 \mathrm{E}-09$ & $1.27 \mathrm{E}+02$ & $\mathbf{6 . 9 9}$ & $2.33 \mathrm{E}-09$ & $1.27 \mathrm{E}+02$ & $\mathbf{6 . 9 9}$ \\
\hline
\end{tabular}

Table 4.5. Errors of the numerical solution from WUCS when $u_{0}(x)=\sin (80 x)$ at $t_{k}=1$

\begin{tabular}{||c|cccccccccc||}
\hline \hline $\mathbf{N}$ & $\begin{array}{c}\text { L } \infty \\
\text { ERROR }\end{array}$ & H/2H & $\begin{array}{c}\text { L } \infty \\
\text { ORDER }\end{array}$ & $\begin{array}{c}\text { L1 } \\
\text { ERROR }\end{array}$ & H/2H & $\begin{array}{c}\text { L1 } \\
\text { ORDER }\end{array}$ & $\begin{array}{c}\text { L2 } \\
\text { ERROR }\end{array}$ & H/2H & $\begin{array}{c}\text { L2 } \\
\text { ORDER }\end{array}$ \\
\hline $\mathbf{3 2 0}$ & $4.46 \mathrm{E}-01$ & $0.00 \mathrm{E}+00$ & & $2.76 \mathrm{E}+00$ & $0.00 \mathrm{E}+00$ & & $1.10 \mathrm{E}+00$ & & \\
$\mathbf{6 4 0}$ & $4.91 \mathrm{E}-03$ & $9.08 \mathrm{E}+01$ & $\mathbf{6 . 5}$ & $2.09 \mathrm{E}-02$ & $1.32 \mathrm{E}+02$ & $\mathbf{7 . 0 5}$ & $9.07 \mathrm{E}-03$ & $1.22 \mathrm{E}+02$ & $\mathbf{6 . 9 3}$ \\
$\mathbf{1 2 8 0}$ & $4.18 \mathrm{E}-05$ & $1.18 \mathrm{E}+02$ & $\mathbf{6 . 8 8}$ & $1.69 \mathrm{E}-04$ & $1.23 \mathrm{E}+02$ & $\mathbf{6 . 9 5}$ & $7.46 \mathrm{E}-05$ & $1.22 \mathrm{E}+02$ & $\mathbf{6 . 9 3}$ \\
$\mathbf{2 5 6 0}$ & $3.34 \mathrm{E}-07$ & $1.25 \mathrm{E}+02$ & $\mathbf{6 . 9 7}$ & $1.34 \mathrm{E}-06$ & $1.26 \mathrm{E}+02$ & $\mathbf{6 . 9 8}$ & $5.93 \mathrm{E}-07$ & $1.26 \mathrm{E}+02$ & $\mathbf{6 . 9 8}$ \\
\hline $\mathbf{5 1 2 0}$ & $2.63 \mathrm{E}-09$ & $1.27 \mathrm{E}+02$ & $\mathbf{6 . 9 9}$ & $1.05 \mathrm{E}-08$ & $1.27 \mathrm{E}+02$ & $\mathbf{6 . 9 9}$ & $4.66 \mathrm{E}-09$ & $1.27 \mathrm{E}+02$ & $\mathbf{6 . 9 9}$ \\
\hline
\end{tabular}


Table 4.6. Errors of the numerical solution from WUCS when $u_{0}(x)=\sin (160 x)$ at $t_{k}=1$

\begin{tabular}{||c|cccccccccc||}
\hline $\mathbf{N}$ & $\begin{array}{c}\text { L } \infty \\
\text { ERROR }\end{array}$ & $\mathbf{H} / \mathbf{2 H}$ & $\begin{array}{c}\text { L } \infty \\
\text { ORDER }\end{array}$ & $\begin{array}{c}\text { L1 } \\
\text { ERROR }\end{array}$ & H/2H & $\begin{array}{c}\text { L1 } \\
\text { ORDER }\end{array}$ & $\begin{array}{c}\text { L2 } \\
\text { ERROR }\end{array}$ & H/2H & $\begin{array}{c}\text { L2 } \\
\text { ORDER }\end{array}$ \\
\hline $\mathbf{6 4 0}$ & $8.92 \mathrm{E}-01$ & & & $5.53 \mathrm{E}+00$ & & & $2.21 \mathrm{E}+00$ & & \\
$\mathbf{1 2 8 0}$ & $9.83 \mathrm{E}-03$ & $9.08 \mathrm{E}+01$ & $\mathbf{6 . 5}$ & $4.18 \mathrm{E}-02$ & $1.32 \mathrm{E}+02$ & $\mathbf{7 . 0 5}$ & $1.81 \mathrm{E}-02$ & $1.22 \mathrm{E}+02$ & $\mathbf{6 . 9 3}$ \\
$\mathbf{2 5 6 0}$ & $8.36 \mathrm{E}-05$ & $1.18 \mathrm{E}+02$ & $\mathbf{6 . 8 8}$ & $3.38 \mathrm{E}-04$ & $1.24 \mathrm{E}+02$ & $\mathbf{6 . 9 5}$ & $1.49 \mathrm{E}-04$ & $1.22 \mathrm{E}+02$ & $\mathbf{6 . 9 3}$ \\
$\mathbf{5 1 2 0}$ & $6.68 \mathrm{E}-07$ & $1.25 \mathrm{E}+02$ & $\mathbf{6 . 9 7}$ & $2.68 \mathrm{E}-06$ & $1.26 \mathrm{E}+02$ & $\mathbf{6 . 9 8}$ & $1.19 \mathrm{E}-06$ & $1.26 \mathrm{E}+02$ & $\mathbf{6 . 9 8}$ \\
$\mathbf{1 0 2 4 0}$ & $5.29 \mathrm{E}-09$ & $1.26 \mathrm{E}+02$ & $\mathbf{6 . 9 8}$ & $2.10 \mathrm{E}-08$ & $1.27 \mathrm{E}+02$ & $\mathbf{6 . 9 9}$ & $9.31 \mathrm{E}-09$ & $1.27 \mathrm{E}+02$ & $\mathbf{6 . 9 9}$ \\
\hline \hline
\end{tabular}
4.1.3 $u_{0}(\mathrm{x})=\left\{\begin{array}{l}0, \\ 1,\end{array}\right.$
if $0 \leq x \leq 1$
if $1<x \leq 2$

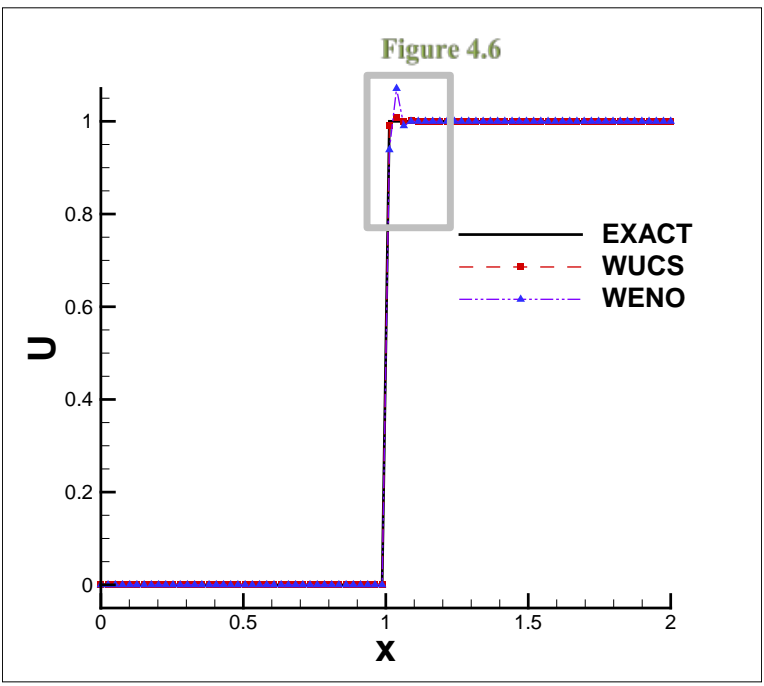

Figure 4.5. The exact solution compared with the numerical solutions from both WUCS and the WENO scheme with $N=40$.

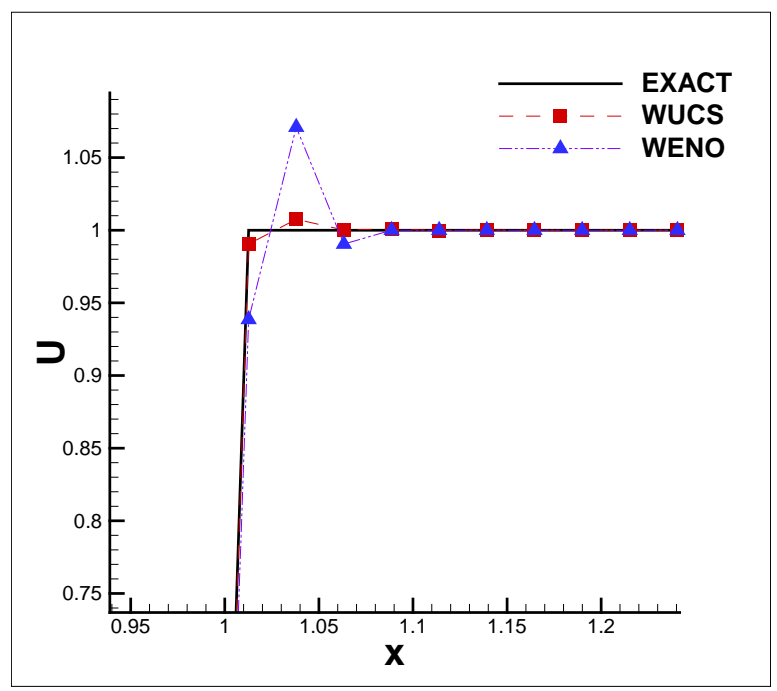

Figure 4.6. The enlarged portion of the selected part as indicated in Figure 4.5. 
4.1.4 $u_{0}(\mathrm{x})= \begin{cases}1, & \text { if }-\frac{1}{4} \leq x \leq \frac{1}{4} \\ 0, & \text { otherwise }\end{cases}$

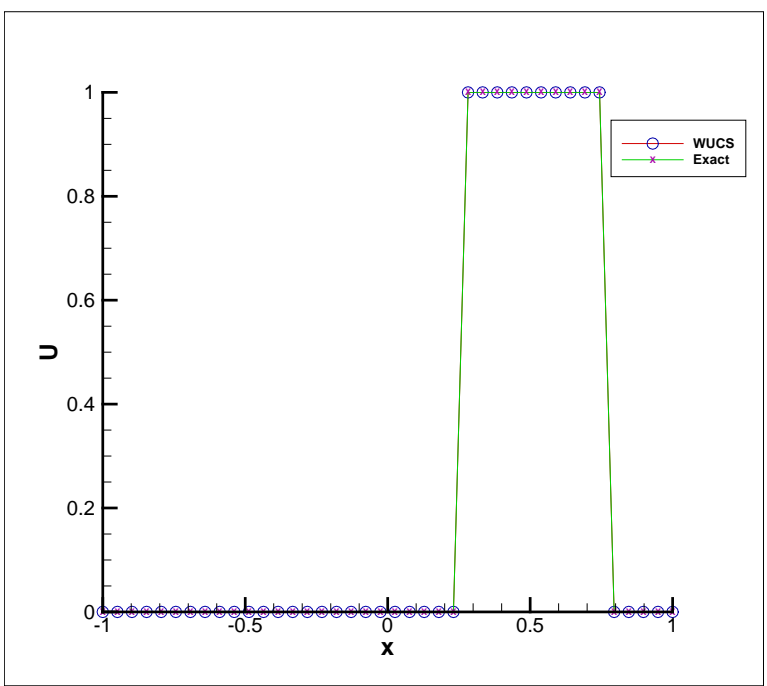

Figure 4.7. The exact solution compared with the numerical solution from WUCS.

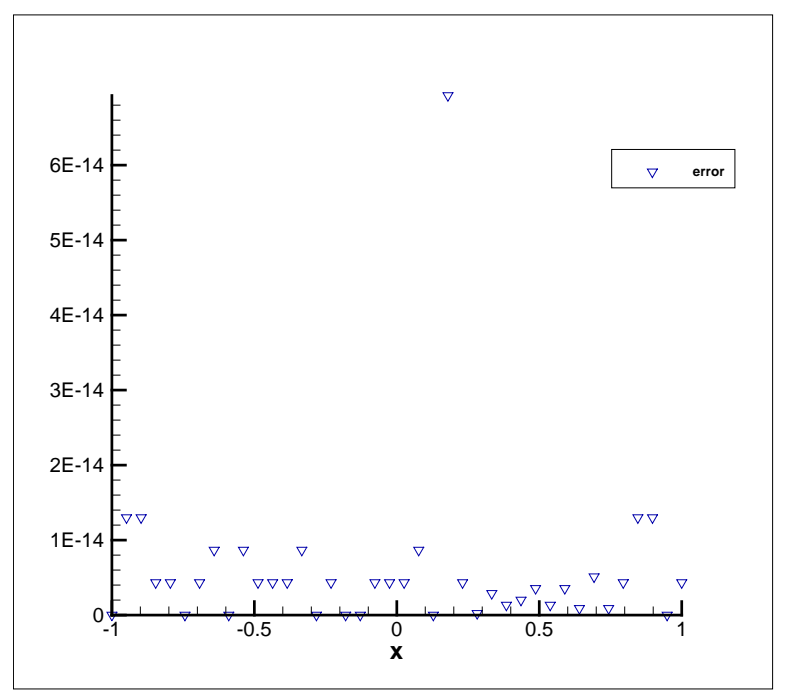

Figure 4.8. Errors of the numerical solution from WUCS at $t_{k}=0.5$ with $N=40$.

\subsection{Burgers’ Equation}

WUCS is used to solve the nonlinear Burgers' equation

$$
u_{t}+u u_{x}=0, \quad \text { where } \quad \mathrm{a}<x<\mathrm{b}
$$

with different initial functions as shown in Figures 4.9 - 4.12. Table 4.7 proves that the scheme has a $7^{\text {th }}$ order of accuracy. 


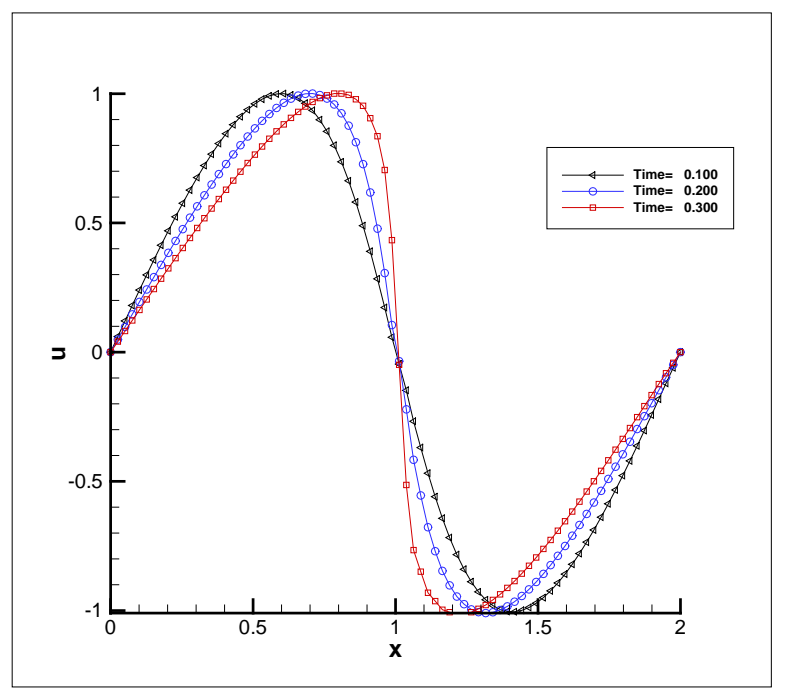

Figure 4.9. The numerical solution from WUCS when $u_{0}(x)=\sin (\pi x)$ at different time steps.

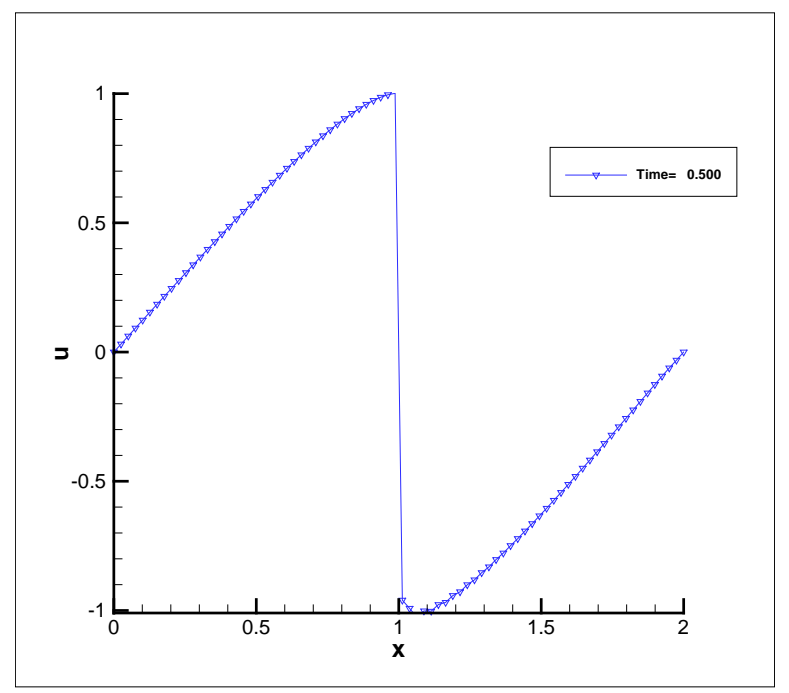

Figure 4.10. The numerical solution from WUCS when $u_{0}(x)=\sin (\pi x)$ at $t_{k}=0.5$.

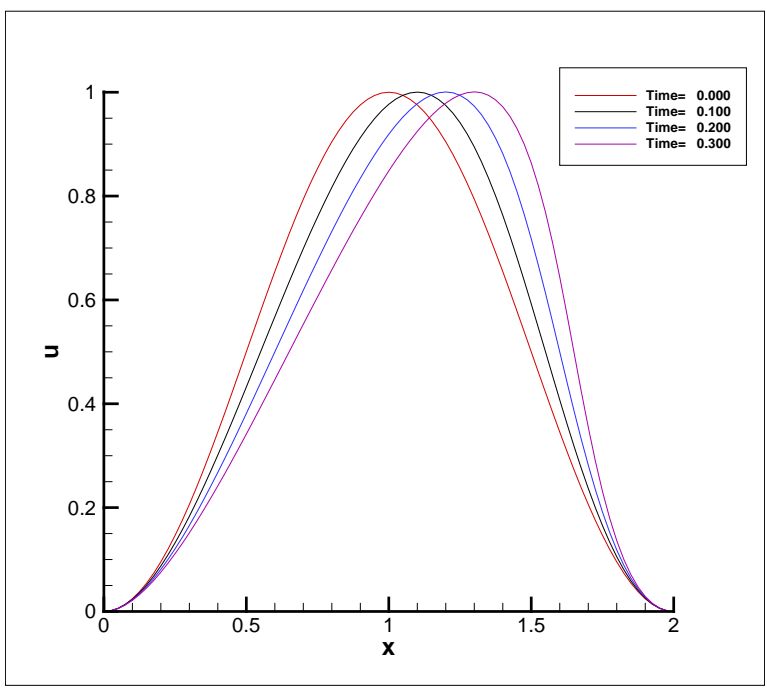

Figure 4.11. The numerical solution from WUCS when $u(x, 0)=\frac{1}{2}(1-\cos (\pi x))$ at different time steps.

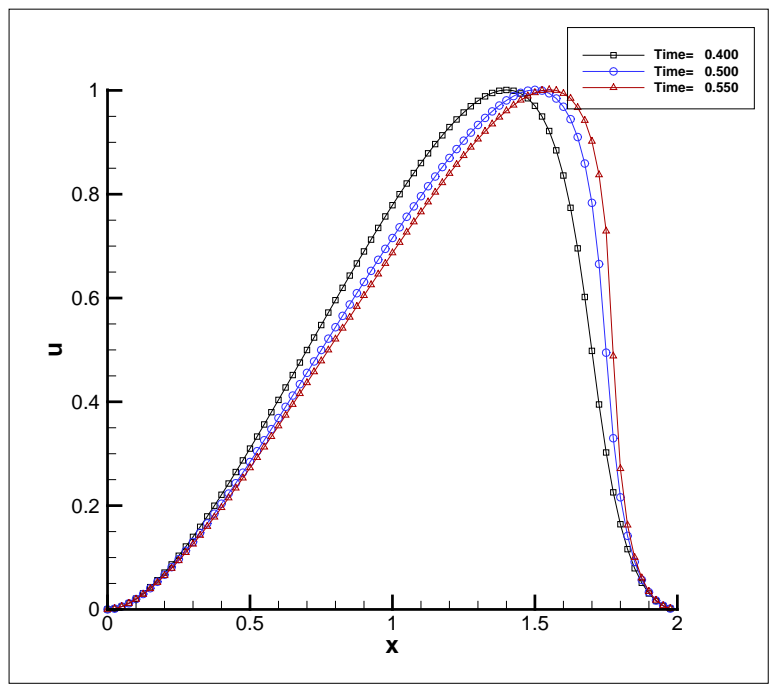

Figure 4.12. The numerical solution from WUCS when $u(x, 0)=\frac{1}{2}(1-\cos (\pi x))$ at different time steps. 
Table 4.7. Errors of the numerical solution obtained by WUCS of Burgers' equation when $u_{0}(x)=\sin (\pi x), 0<x<1$ at $t=1$

\begin{tabular}{|c|c|c|c|c|c|c|c|c|c|}
\hline $\mathbf{N}$ & $\begin{array}{c}\text { L } \infty \\
\text { ERROR }\end{array}$ & $\mathbf{H} / 2 \mathbf{H}$ & $\begin{array}{c}\text { L } \infty \\
\text { ORDER }\end{array}$ & $\begin{array}{c}\text { L1 } \\
\text { ERROR }\end{array}$ & $\mathbf{H} / 2 \mathbf{H}$ & $\begin{array}{c}\text { L1 } \\
\text { ORDER }\end{array}$ & $\begin{array}{c}\text { L2 } \\
\text { ERROR }\end{array}$ & $\mathbf{H} / 2 \mathbf{H}$ & $\begin{array}{c}\text { L2 } \\
\text { ORDER }\end{array}$ \\
\hline 8 & $1.15 \mathrm{E}-01$ & & & $1.44 \mathrm{E}-02$ & & & 4.07E-02 & & \\
\hline 16 & $2.23 \mathrm{E}-03$ & $5.15 E+01$ & 5.69 & 2.09E-04 & $6.88 \mathrm{E}+01$ & 6.15 & $5.80 \mathrm{E}-04$ & $7.01 \mathrm{E}+01$ & 6.14 \\
\hline 32 & $1.28 \mathrm{E}-05$ & $1.74 \mathrm{E}+02$ & 7.44 & $1.50 \mathrm{E}-06$ & $1.39 \mathrm{E}+02$ & 7.12 & 3.07E-06 & $1.89 \mathrm{E}+02$ & 7.56 \\
\hline 64 & $1.42 \mathrm{E}-07$ & $8.99 \mathrm{E}+01$ & 6.49 & 9.65E-09 & $1.55 \mathrm{E}+02$ & 7.27 & $3.08 \mathrm{E}-08$ & $9.98 \mathrm{E}+01$ & 6.65 \\
\hline 128 & 1.29E-09 & $1.10 \mathrm{E}+02$ & 6.79 & 8.10E-11 & $1.19 \mathrm{E}+02$ & 6.90 & 2.61E-10 & $1.18 \mathrm{E}+02$ & 6.88 \\
\hline
\end{tabular}

\subsection{Euler Equations}

The typical one-dimensional Euler equations for gas dynamics in strong conservation form are

$$
\begin{aligned}
& \frac{\partial \mathrm{U}}{\partial \mathrm{t}}+\frac{\partial \mathrm{F}}{\partial \mathrm{x}}=0 \quad \text { where } \\
& \mathrm{U}=\left[\begin{array}{c}
\rho \\
\rho u \\
E
\end{array}\right] \text {, and } \\
& \mathrm{F}=\left[\begin{array}{c}
\rho u \\
\rho u^{2}+P \\
(E+P) u
\end{array}\right]
\end{aligned}
$$

where $\rho, u, P, E$ are the density, velocity, pressure, and total energy, respectively.

The equation of the state is given by

$$
\mathrm{P}=(\gamma-1)\left(E-\frac{1}{2} \rho u^{2}\right) \text { where } \quad \gamma=1.4
$$


To improve the shock-capturing property of the Modified Weighted Compact Scheme (MWCS) (H. Fu et al., 2013), the idea of decoupling the system of the Weighted Upwinding Compact Scheme (WUCS (ID)) is used in MWCS to solve the onedimensional Euler equations with different initial conditions. The first example is the shock tube problem, and the initial conditions are chosen in a way such that the shock, contact discontinuity, and a rarefaction will occur in the solution. The second example, shock-entropy interaction, simulates the interaction between the shock wave and fluctuations. To solve the Euler equations, three-step TVD Runge-Kutta is used in time marching. Additionally, Lax-Friedrich flux vector splitting is used in solving the Euler equations.

\subsubsection{Shock tube problem}

First, the one-dimensional Euler equations above are solved with the following initial conditions

$$
(\rho, u, P)=\left\{\begin{array}{c}
(1.0,0,1.0), \quad x \leq 0 \\
(0.125,0,0.1), \quad x>0
\end{array}\right.
$$

Figures 4.13 - 4.20 illustrate the distribution of the pressure, density, velocity, and energy calculated by the decoupling method of the proposed scheme and compared with the related schemes, the WENO scheme and MWCS, when $\mathrm{N}=221$. These figures show the ability of the scheme to capture the shock wave and the contact discontinuities. In the following figures, the solid line represents the numerical solution obtained by the WENO scheme with a fine grid of $\mathrm{N}=1601$, and this is regarded as an exact solution. 


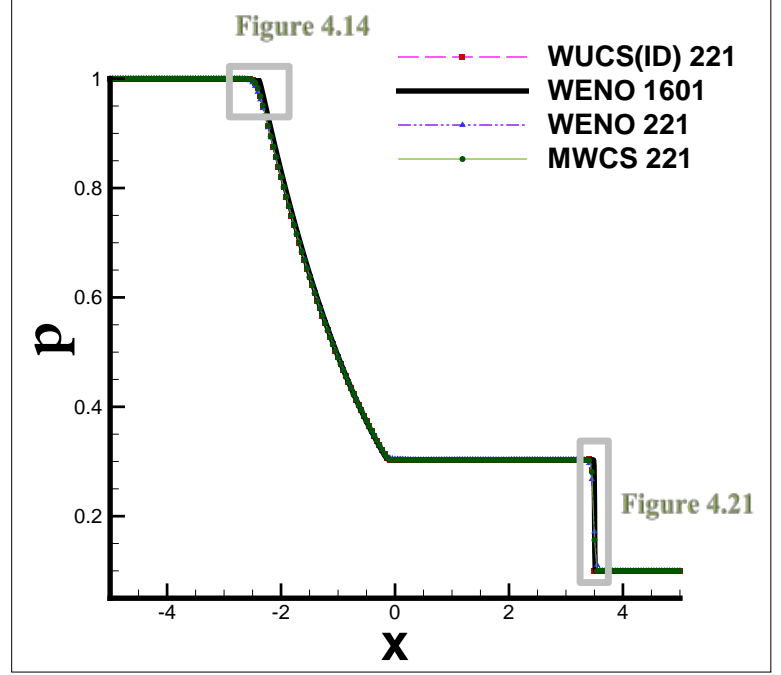

Figure 4.13. The shock tube problem. Distribution of pressure at $t=2$.

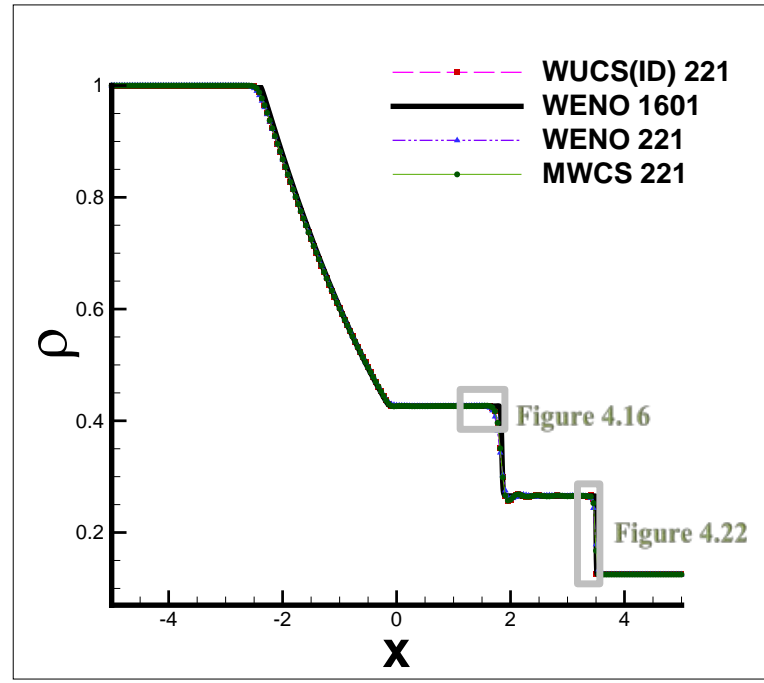

Figure 4.15. The shock tube problem. Distribution of densitv solution at $t=2$.

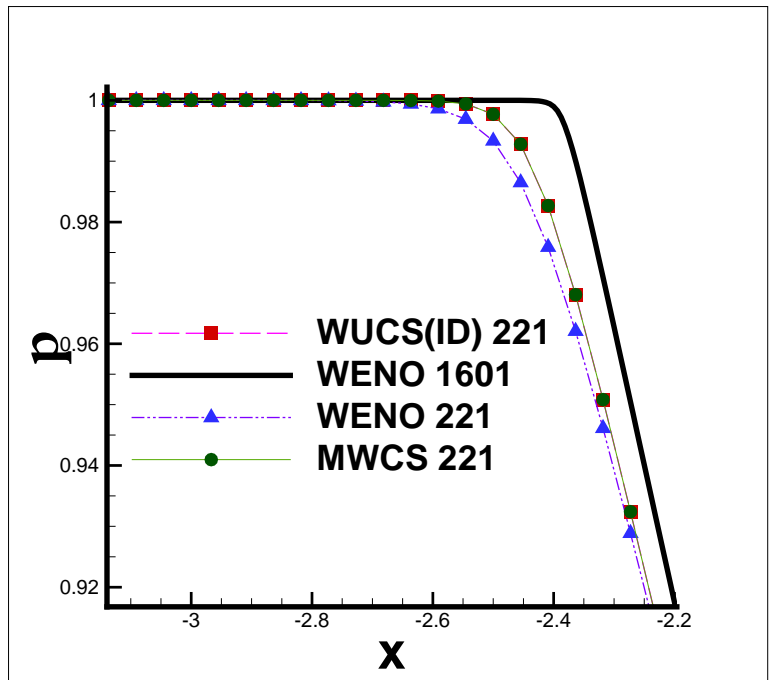

Figure 4.14. The enlarged portion of the selected part as indicated in Figure 4.13.

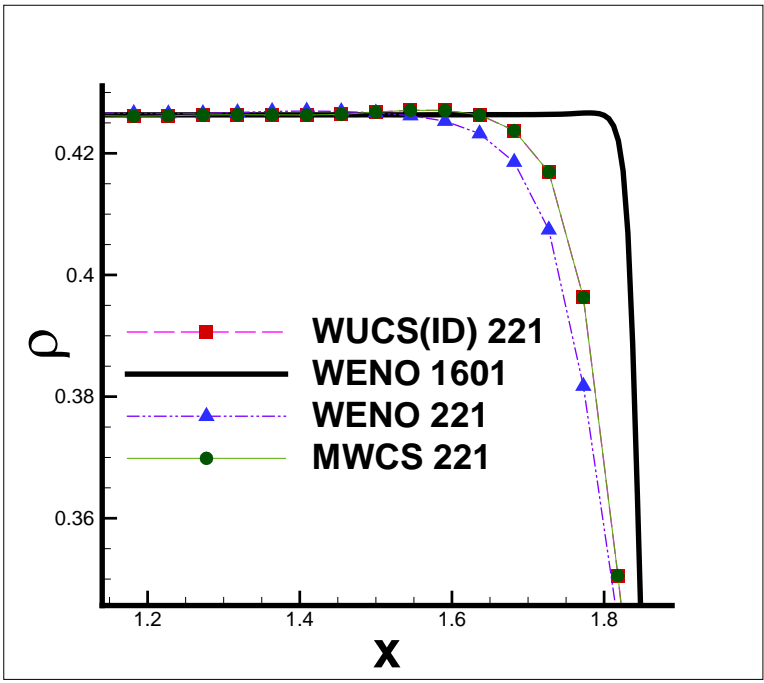

Figure 4.16. The enlarged portion of the selected part as indicated in Figure 4.15. 


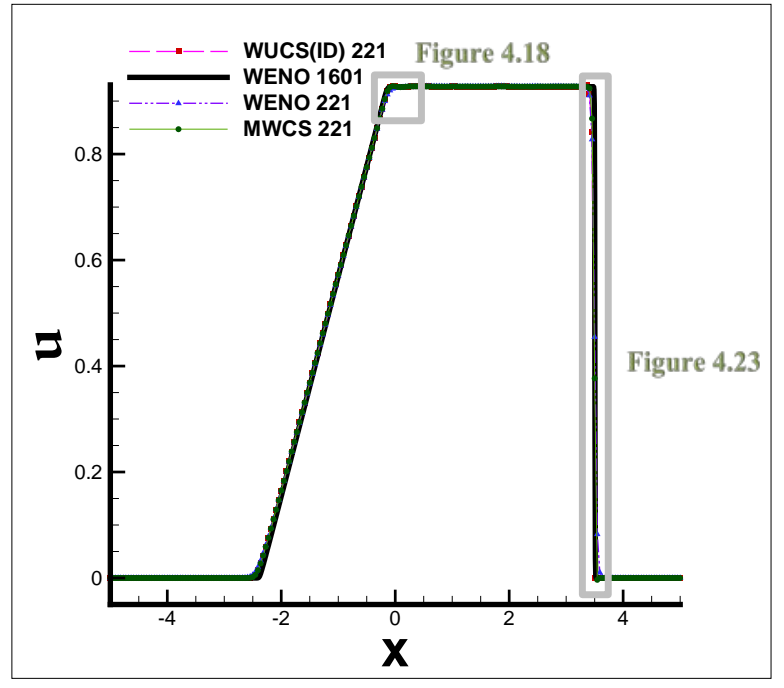

Figure 4.17. The shock tube problem. Distribution of velocity at $t=2$.

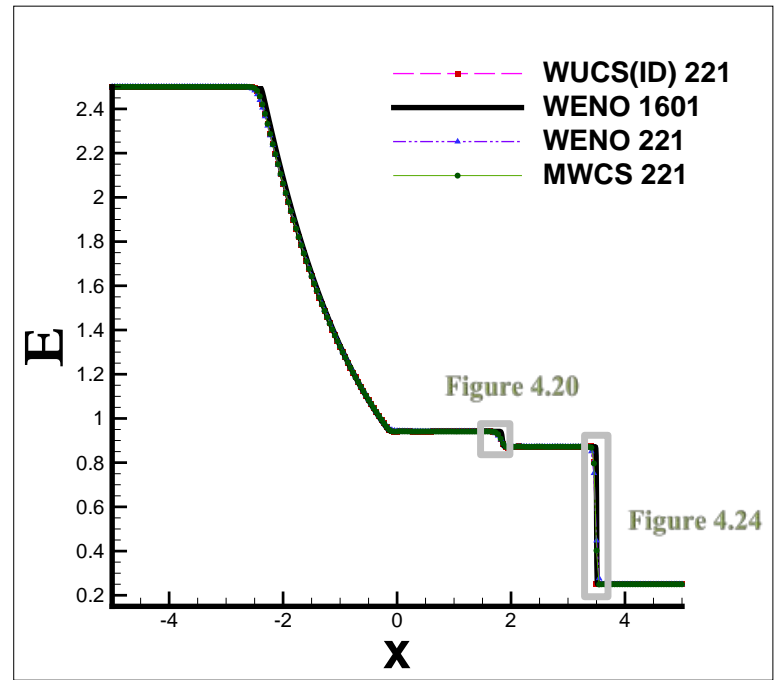

Figure 4.19. The shock tube problem. Distribution of energy at $t=2$.

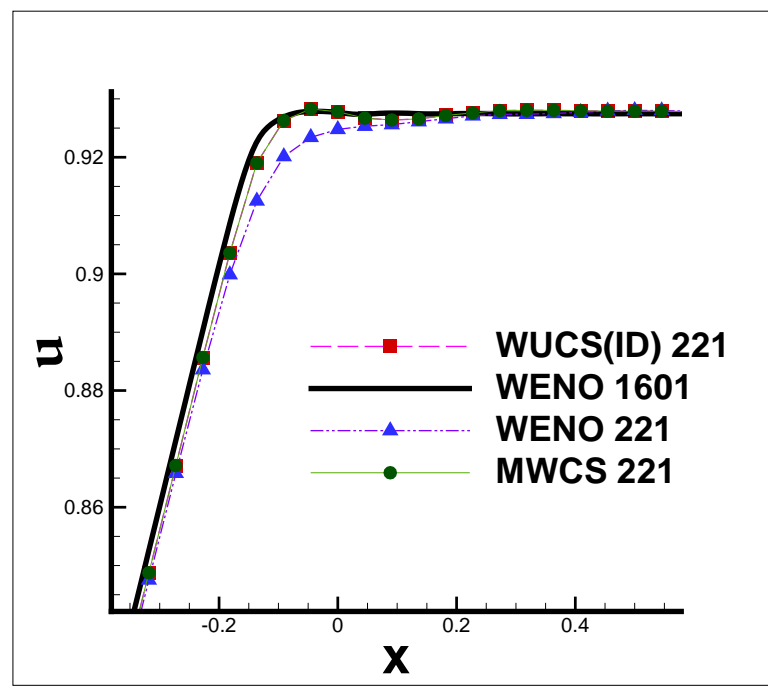

Figure 4.18. The enlarged portion of the selected part as indicated in Figure 4.17.

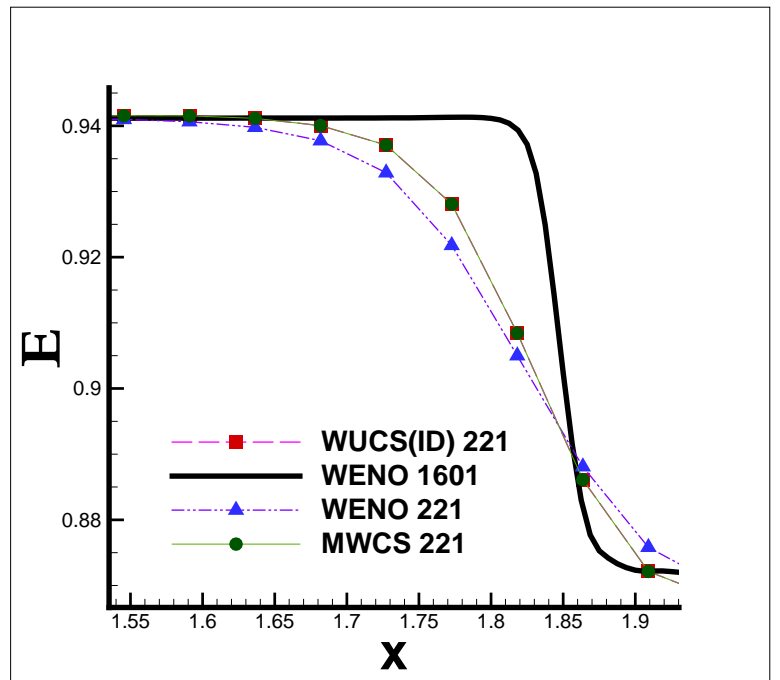

Figure 4.20. The enlarged portion of the selected part as indicated in Figure 4.19. 
Figures 4.21-4.24 clearly indicate the preference of WUCS(ID) near shock areas compared with the WENO scheme and MWCS because it requires fewer points to capture the shocks.

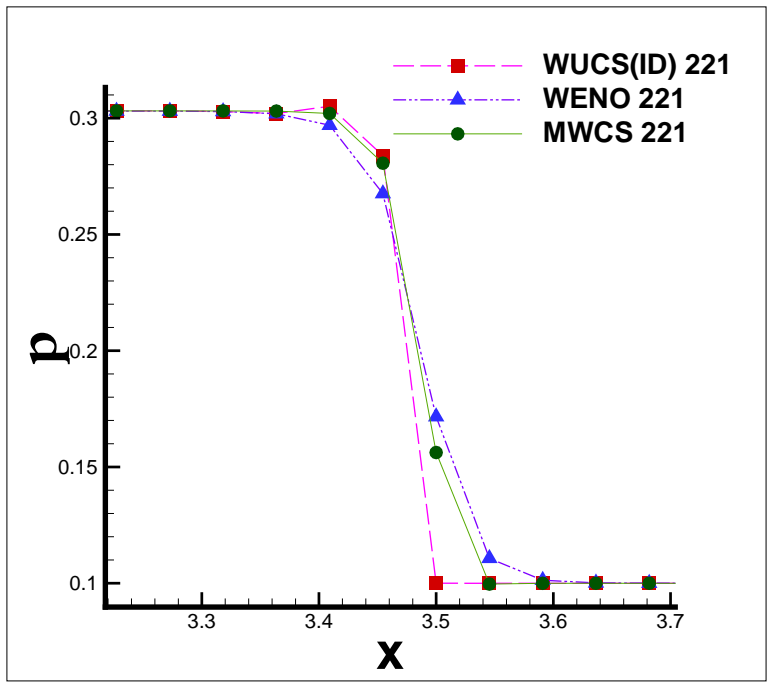

Figure 4.21. The shock tube problem. The enlarged portion of the selected part as indicated in Figure 4.13.

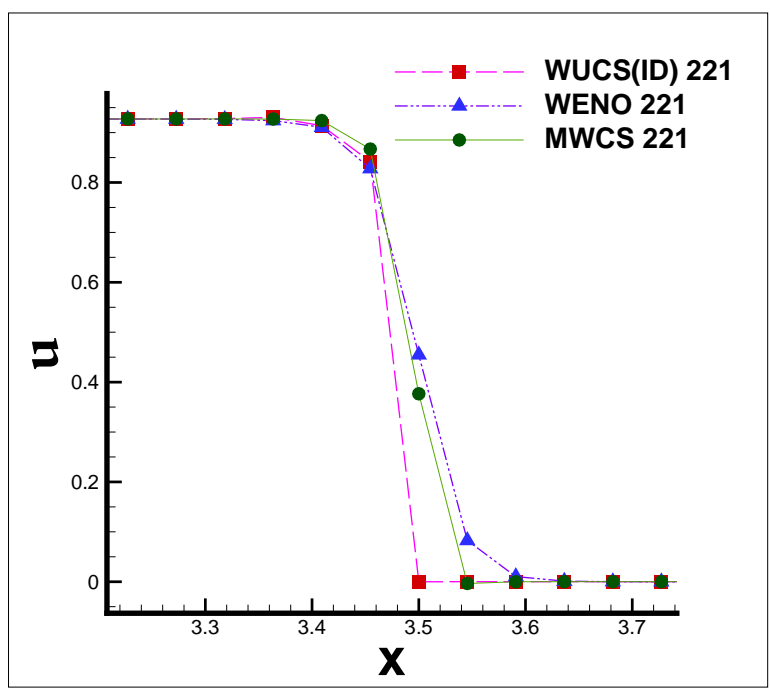

Figure 4.23. The shock tube problem. The enlarged portion of the selected part as indicated in Figure 4.17.

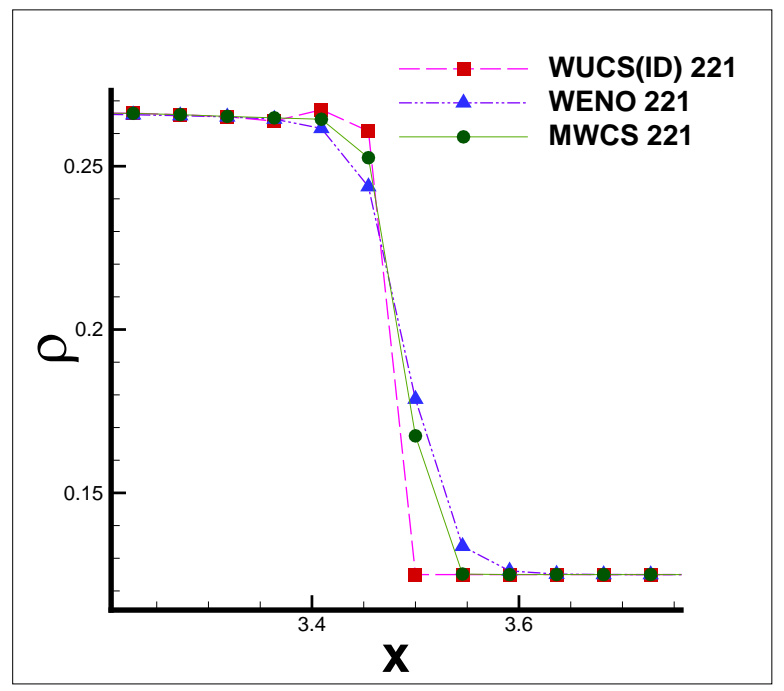

Figure 4.22. The shock tube problem. The enlarged portion of the selected part as indicated in Figure 4.15.

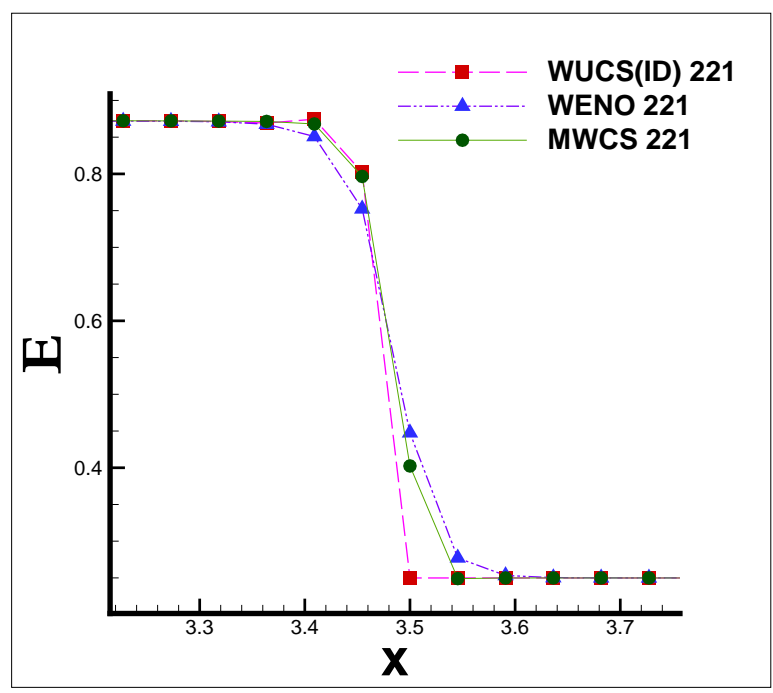

Figure 4.24. The shock tube problem. The enlarged portion of the selected part as indicated in Figure 4.19. 


\subsubsection{Shock-entropy interaction problem}

To test the shock-capturing capacity and resolution, the scheme is applied to the 1-D problem of shock-entropy wave interaction. In this case, the Euler equations are solved with the following initial conditions:

$$
(\rho, u, P)=\left\{\begin{array}{cc}
(3.857143,2.629369,10.33333), & x<-4 \\
(1+0.2 \sin (5 x), 0,1), & x \geq-4
\end{array}\right.
$$

The shock in this problem is moving into a density fluctuation field, and it interacts with the fluctuation. The results from WUCS(ID) are compared with the WENO scheme and MWCS as shown in Figures 4.25 - 4.32. It can be clearly seen how the fine structures are resolved with high frequency by the presented scheme without serious oscillation near shock areas.

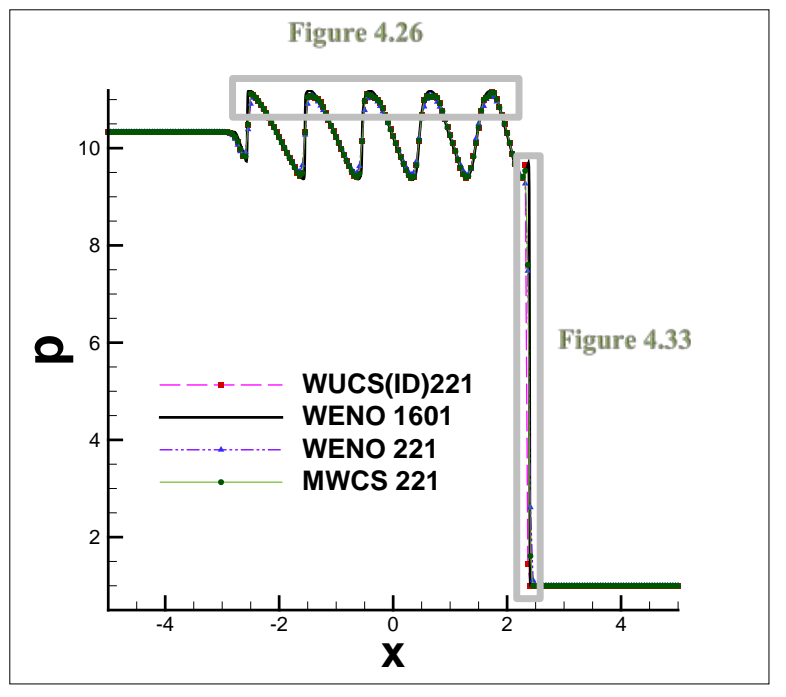

Figure 4.25. The shock-entropy interaction problem. Distribution of pressure at $t=1.8$.

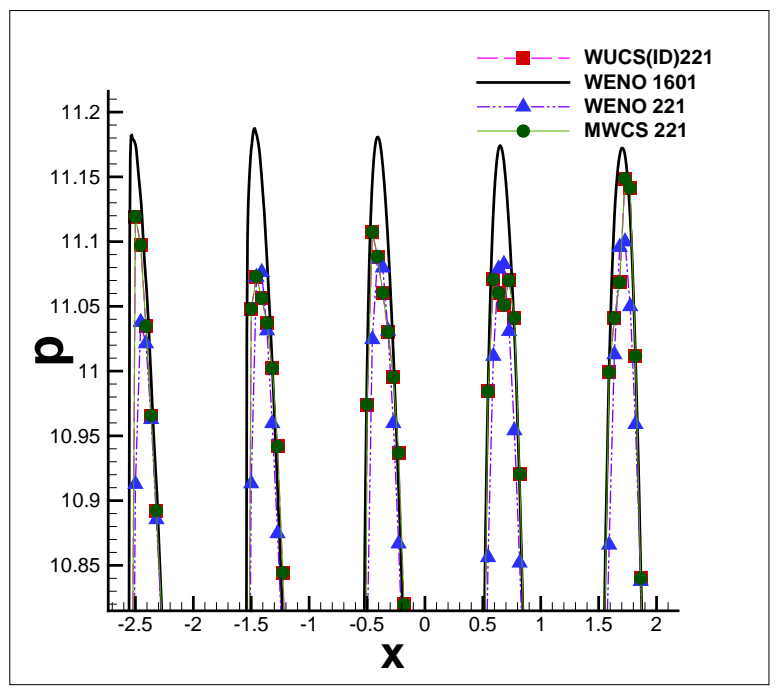

Figure 4.26. The enlarged portion of the selected part as indicated in Figure 4.25. 


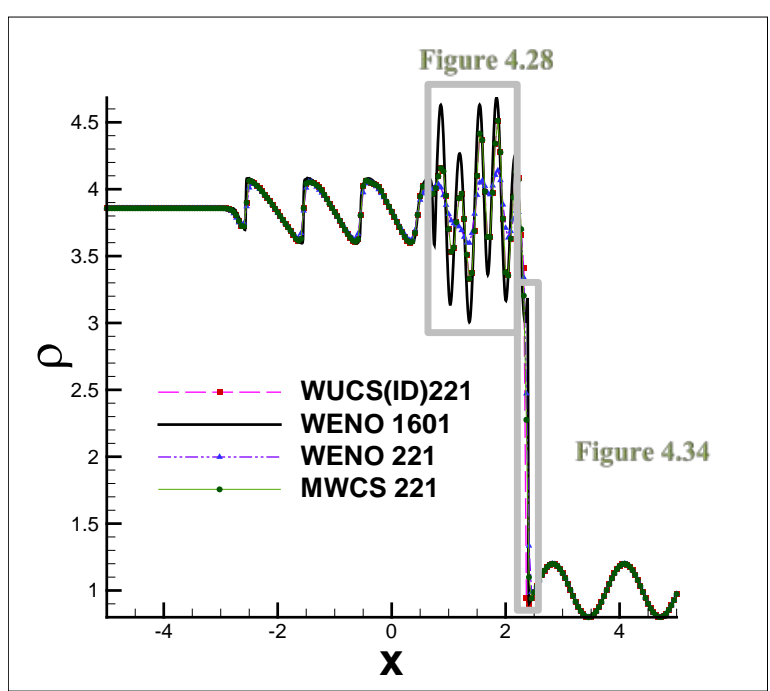

Figure 4.27. The shock-entropy interaction problem. Distribution of density at $t=1.8$.

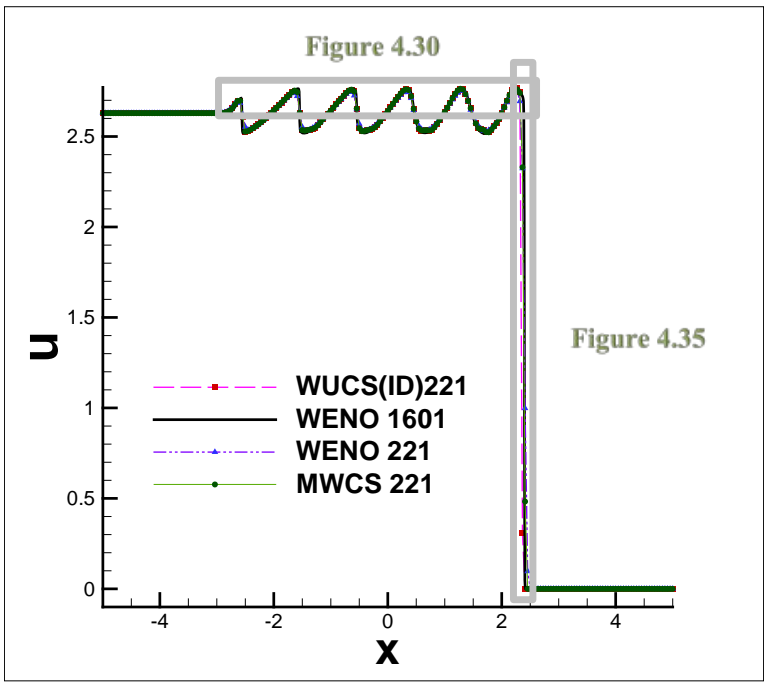

Figure 4.29. The shock-entropy interaction problem. Distribution of velocity at $t=1.8$.

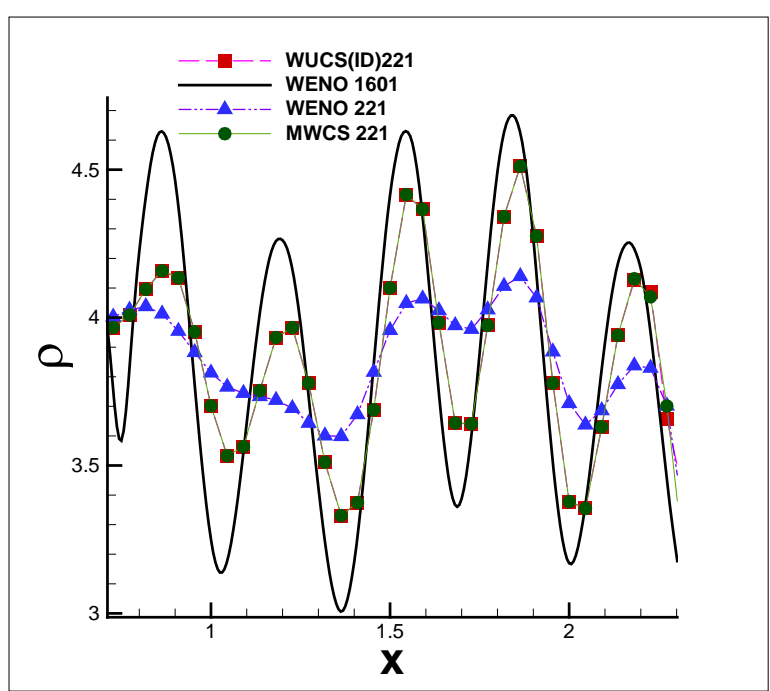

Figure 4.28. The enlarged portion of the selected part as indicated in Figure 4.27.

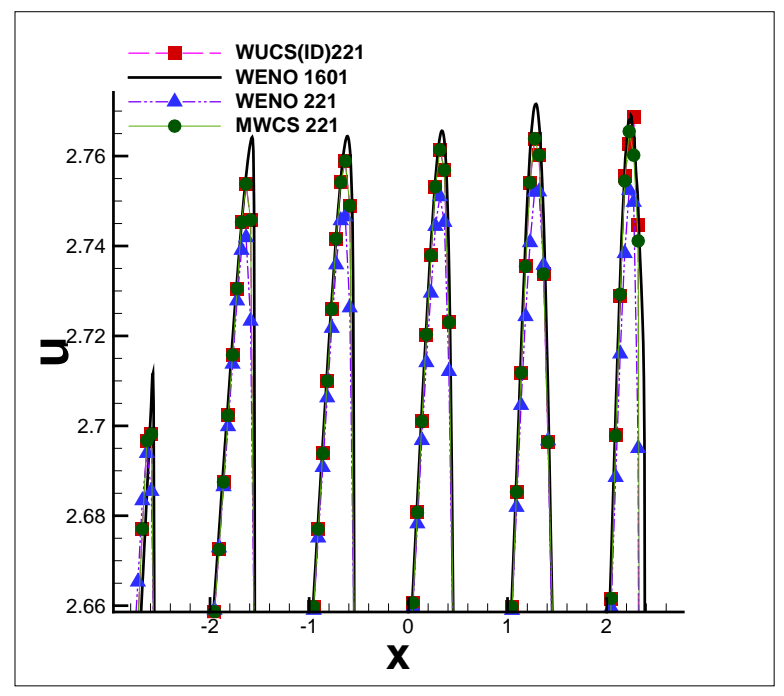

Figure 4.30. The enlarged portion of the selected part as indicated in Figure 4.29. 


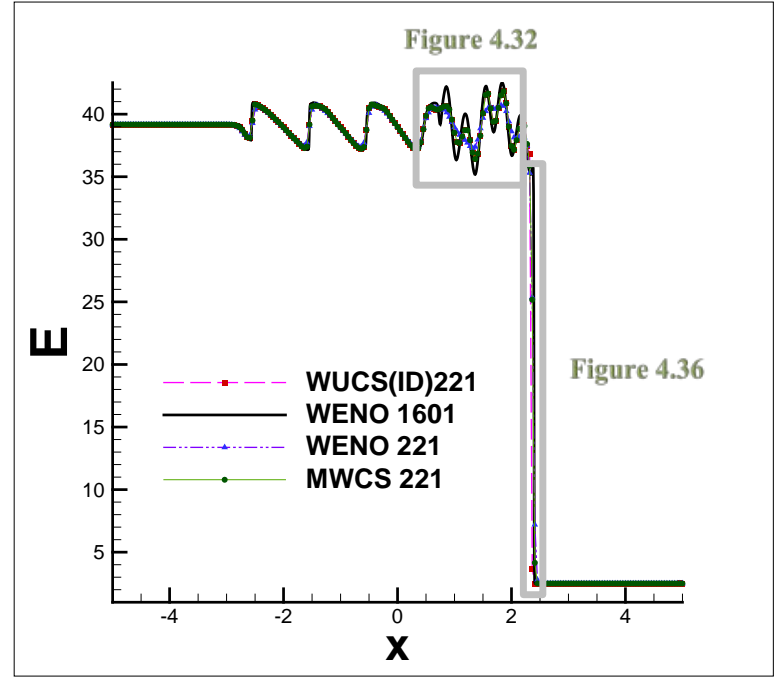

Figure 4.31. The shock-entropy interaction problem. Distribution of energy at $t=1.8$.

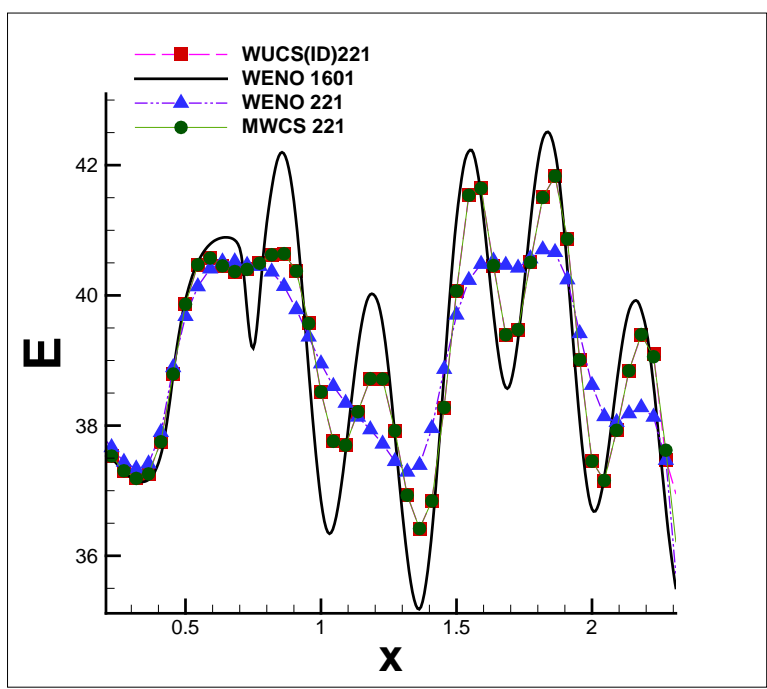

Figure 4.32. The enlarged portion of the selected part as indicated in Figure 4.31.

In Figures 4.33-4.36, the results from WUCS(ID) are compared with WENO and MWCS schemes near shock areas. From these figures, one can see the ability of the proposed scheme to capture the shock. Moreover, the results from WUCS(ID) are sharper and much better than the WENO scheme and MWCS in the shock areas as shown below because fewer points are required to capture the discontinuity compared to the WENO scheme and MWCS. 


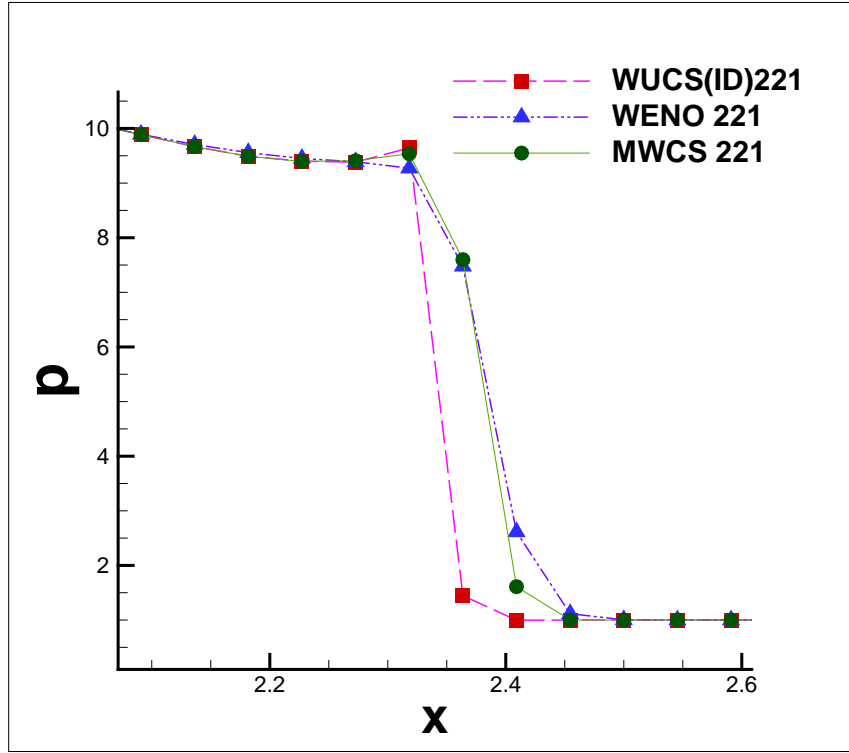

Figure 4.33. The shock-entropy interaction problem. The enlarged portion of the selected part as indicated in Figure 4.25.

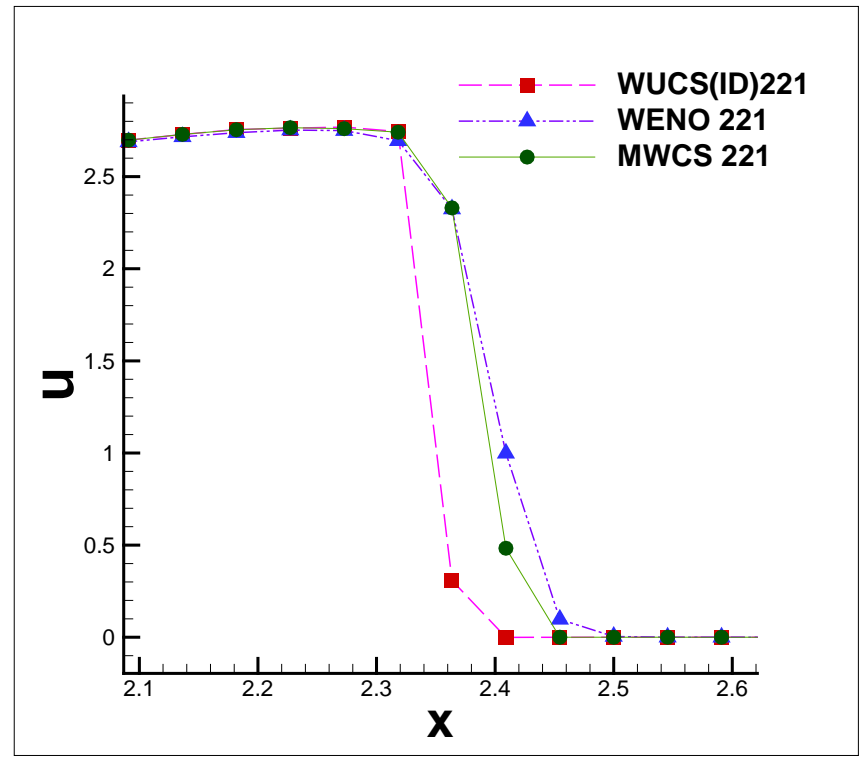

Figure 4.35. The shock-entropy interaction problem. The enlarged portion of the selected part as indicated in Figure 4.29.

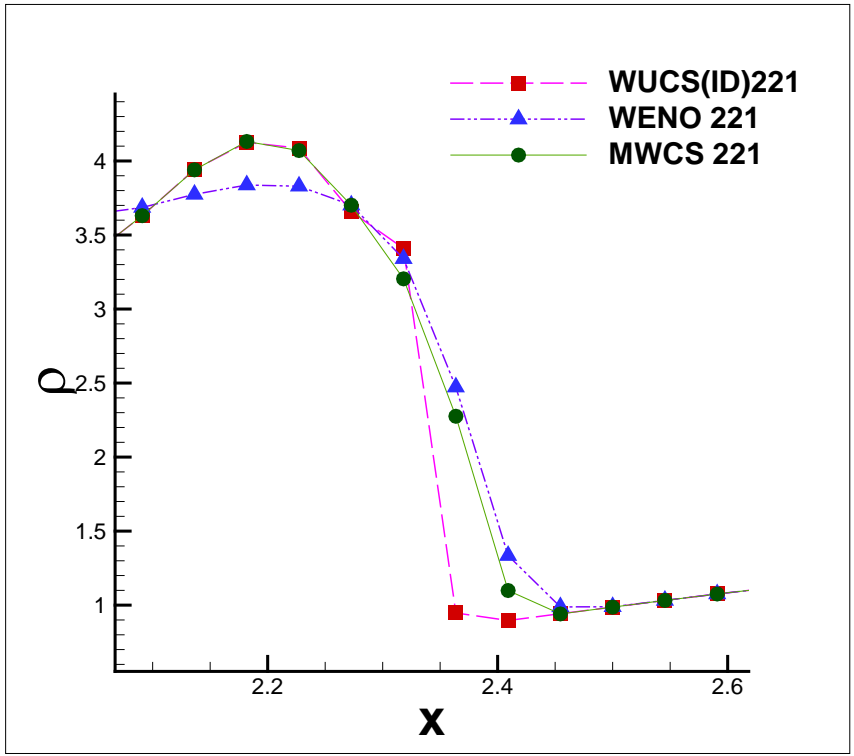

Figure 4.34. The shock-entropy interaction problem. The enlarged portion of the selected part as indicated in Figure 4.27.

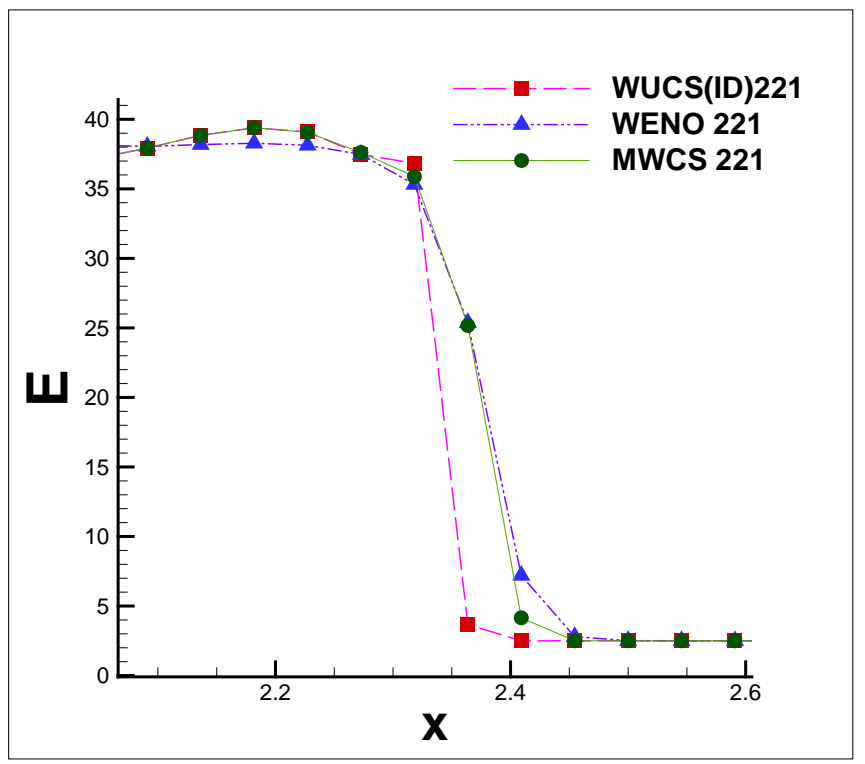

Figure 4.36. The shock-entropy interaction problem. The enlarged portion of the selected part as indicated in Figure 4.31. 


\subsection{Conclusion}

WUCS is applied to many one dimensional typical problems involving discontinuities and shock waves in this chapter. The results show that the proposed scheme has a high order in smooth areas and has the capability to capture the shock by making the global dependence become an upwinding dependence. Many high frequency examples are solved by WUCS to indicate that the scheme has a $7^{\text {th }}$ order of accuracy with high resolution in smooth regions. For non-smooth regions, the system is decoupled into subsystems, and these subsystems are solved using WUCS. In other words, the derivative matrix is decoupled into submatrices according to shock locations. Compared to the WENO scheme and MWCS, the results from WUCS(ID) are much more accurate because the number of points that are required to capture the discontinuity are decreased. 


\section{CHAPTER 5}

\section{CONCLUSION AND FUTURE WORK}

For smooth and periodic functions, the Fast Fourier Transform (FFT) algorithm can be applied successfully, but it is not suitable for non-periodic functions. To overcome this issue, the Modified Buffered Fourier Method (MBFM) is illustrated in this dissertation. The proposed method can deal with non-periodic problems by using the idea of the buffered zone, which can be implemented by extending the domain and adding smooth interpolations. In the Standard Fourier Spectral Method (SFSM), the derivative of the source function might not be periodic on the boundaries, so large oscillation near the boundaries may appear. However, the oscillation will be highly reduced when using MBFM because MBFM can make the function and its derivative periodic on the boundaries. Also, compared with SFSM, the order of accuracy can be improved from 2 to 7 when using MBFM as shown in the numerical examples. Additionally, MBFM requires less computation time compared with other numerical methods because it keeps all the advantages of FFT. Hence, adding only a $25 \%$ buffer zone, a high order solution with much less oscillation near the boundaries will be gained. Moreover, there is an effort to apply the proposed method in some cavity flow problems.

For non-smooth functions, the Weighted Upwinding Compact Scheme (WUCS), which belongs to the family of finite difference schemes, is proposed. 
Based on the analysis of the dissipation and dispersion at each stencil, the upwinding candidates are constructed in such a way that the left and central candidates have a positive dissipation with a very small dispersion for high resolution. In addition, the global dependency problem, using all grid points including the downstream points to get the derivative, is avoided by WUCS because it is prohibited for the shock case. When applying WUCS to many one dimensional typical problems involving discontinuities and shock waves, the results show that the proposed scheme has a $7^{\text {th }}$ order in smooth areas and can capture the shock in the non-smooth areas by decoupling the system into subsystems. Sharper solutions and fewer points in shock regions are achieved from WUCS(ID) compared to the WENO scheme and MWCS.

Many efforts will be made to use the decoupling technology for the new scheme and apply it to:

- All cases of the 1-D Euler equations.

- 2-D and 3-D Navier-Stokes equations

- Multi-dimensional flows with shock-turbulence interaction. 


\section{REFERENCES}

[1] A. Harten, B. Engquist, S. Osher, and S.R. Charkravarthy, Uniformly high order accurate essentially non-oscillatory schemes III, Journal of Computational Physics, 71 (1987), pp. 231-303.

[2] B. Orel, and A. Perne, Chebyshev-Fourier Spectral Methods for Nonperiodic Boundary Value Problems, Journal of Applied Mathematics, 2014 doi:10.1155/2014/572694.

[3] B. Van Leer, Towards the ultimate conservative difference scheme. V: A second-order sequel to Godunov’s scheme, Journal of Computational Physics,32 (1979), pp.101-136.

[4] C. Liu and Y. Wang, New Vortex Identification Method and Vortex Ring Development Analysis in Boundary Layer Transition, 54th AIAA Aerospace Sciences Meeting, January 4-8, 2016, San Diego, California.

[5] C. Liu and Z. Liu, Multigrid mapping and box relaxation for simulation of the whole process of flow transition in 3-D boundary layers, Journal of Computational Physics, Vol. 119 (1995), pp. 325-341.

[6] C. Liu, New Theory on Turbulence Generation and Sustenance, TGS2015 Workshop, Tsinghua University, Beijing, China, June 4-6, 2015.

[7] C. Liu, P. Lu, M. Oliveira, and P. Xie, (2012). Modified Upwinding Compact Scheme for Shock and Shock Boundary Layer Interaction. Communications in Computational Physics, 11(3), 1022-1042. doi:10.4208/cicp.250110.160211a

[8] C. W. Shu and S. Osher, Efficient implementation of essentially non-oscillatory shock capturing scheme, Journal of Computational Physics, 77 (1988), pp. 439-471. 
[9] C. W. Shu and S. Osher, Efficient implementation of essentially non-oscillatory shock capturing schemes II, Journal of Computational Physics, 83 (1989), pp. 32-78.

[10] D. Huybrechs, On the Fourier Extension of Non-periodic Functions, SIAM Journal of Numerical Analysis, Vol. 47 (2010), No. 6, pp. 4326-4355.

[11] G. S. Jiang and C. W. Shu, Efficient implementation of weighted ENO scheme. Journal of Computational Physics, 126 (1996), pp. 202-228.

[12] H. Fu, C. Liu, A Buffered Fourier Spectral Method for Non-periodic PDE, International Journal of Numerical Analysis and Modeling, Vol. 9 (2012), No. 2, pp. 460-478.

[13] H. Fu, Z. Wang, and C. Liu, Modified Weighted Compact Scheme for Shock Capturing, Computers \& Fluids, Vol. 96 (2014), pp. 165-176.

[14] H. Shan, L. Jiang, W. Zhao, and C. Liu, Large eddy simulation of flow transition in a supersonic flat-plate boundary layers, AIAA Aerospace Sciences Meeting, 1999, paper 99-0425.

[15] J. P. Boyd, Chebychev and Fourier Spectral Method, 2nd ed., Dover Publications, Inc., 2000.

[16] J. Shen, Efficient Spectral-Galerkin method I. Direct Solvers of Second and Fourthorder Equations Using Legendre Polynomials, SIAM Journal on Scientific Computing, 1994 , doi:10.1137/0915089.

[17] L. Jiang, H. Shan, and C. Liu, Weighted Compact Scheme for Shock Capturing, International Journal of Computational Fluid Dynamics, Vol. 15 (2001), pp. 147-155.

[18] M. Oliveira and C. Liu, New Shock Detector for Shock Boundary Layer Interaction, Lecture Notes in Computer Science, High Performance Computing and Applications, Springer, ISSN 0302-9743, pp.78-87, March 2010. 
[19] M. P. Martin, Direct numerical simulation of hypersonic turbulent boundary layers. Part 1. Initialization and Comparison with Experiments, Journal of Fluid Mechanics, 570 (2007), pp. 347-364.

[20] M. Visbal and D. Gaitonde, On the use of higher-order finite-difference schemes on curvilinear and deforming meshes, Journal of Computational Physics, 181 (2002), pp. $155-185$.

[21] P. Lu, M. Oliveira, and C. Liu, High order compact scheme for Dirichlet boundary points, Neural, Parallel and Scientific Computations, 16 (2) (2008), pp. 273-282.

[22] P. L. Roe, Approximate Riemann solvers, parameter vectors and difference schemes, Journal of Computational Physics, 43 (1981), pp. 357-372.

[23] P. Lu, M. Oliveira, C. Liu, High-order compact scheme for boundary points, International Journal of Computer Mathematics,87 (8) (2008), pp. 1795-1819, doi: 10.1080/00207160802506512.

[24] R. L. Burden, J.D. Faires, Numerical Analysis, 7th ed., Thomson Learning, Inc., 2001.

[25] R. Vichnevetsky and J. B. Bowles, Fourier analysis of numerical approximations of hyperbolic equations. Philadelphia: SIAM, 1982.

[26] S. K. Godunov, A difference scheme for numerical computation of discontinuous solution of hydrodynamic equations, Math Sbornik 47 (1969), pp. 271-306 (in Russian) translated US Joint Publ. Res. Service, JPRS 7226.

[27] S. K. Lele, Compact finite difference schemes with spectral-like resolution, Journal of Computational Physics, 103 (1992), pp. 16-42. 
[28] S. K. Richards, X. Zhang, and X. X. Chen, P.A. Nelson, The Evaluation of Nonreflecting Boundary Conditions for Duct Acoustic Computation, Journal of Sound and Vibration 270, p. 539-557, 2004.

[29] X. D. Liu, S. Osher, and T. Chan, Weighted essentially non-oscillatory schemes, Journal of Computational Physics, 115 (1994), pp. 200-212.

[30] X. Huang, and X. Zhang, A Fourier Pseudo-spectral Method for Some Computational Aeroacoustics Problems, International Journal of Aeroacoustics, Vol. 5 (2006), No. 3, pp. 279-294.

[31] Y. Agnon and H. B. Bingham, A Non-periodic Spectral Method with Application to Nonlinear Water Waves, European Journal of Mechanics / B Fluids, 18(3), 527-534, 1999, doi:10.1016/S0997-7546(99)80047-8.

[32] Y. Wang, H. Al-Dujaly, J. Tang, and C. Liu, (2015). DNS study on hairpin vortex structure in turbulence, 53rd AIAA Aerospace Sciences Meeting. American Institute of Aeronautics and Astronautics Inc, AIAA. doi: 10.2514/6.2015-1524.

[33] Y. Yang, J. Tang, and C. Liu, (2016). LES Analysis on Shock-Vortex Ring Interaction. 54th AIAA Aerospace Sciences Meeting (pp. 1-13). San Diego, California: American Institute of Aeronautics and Astronautics.

[34] Z. Wang, H. Al-Dujaly, Y. Dong, and C. Liu, Construction Methodology of Weighted Upwind Compact Scheme, 54th AIAA Aerospace Sciences Meeting, AIAA SciTech, AIAA 2016-2061. 


\section{BIOGRAPHICAL INFORMATION}

Hassan Abd Salman Al-Dujaly was born in Aldujail (north Baghdad), Iraq. He has received a Bachelor of Science degree in mathematics (2001) and a Master of Science degree in applied mathematics (2008) from the University of Baghdad in Iraq. Also, he has received a High Diploma degree in Computer Science and Informatics (2002) from the Institute for Post Graduate Studies in Informatics in Iraq.

His Master of Science degree in Mathematics - computer science - was conferred in 2015 from the University of Texas at Arlington (UTA), and his PhD degree in mathematics from UTA will be conferred in 2017 upon graduation. His

research interests include numerical methods and their applications in Computational Fluid Dynamics (CFD). 